\title{
Remodeling and Enhancing Schmidt Reaction Pathways in Hexafluoroisopropanol
}

Hashim F. Motiwala, ${ }^{1}$ Manwika Charaschanya, ${ }^{1}$ Victor W. Day, ${ }^{2}$ and Jeffrey Aubé ${ }^{1}$ *

${ }^{1}$ Department of Medicinal Chemistry, University of Kansas, Del Shankel Structural Biology Center, 2034 Becker Drive, Lawrence, Kansas 66047

${ }^{2}$ X-ray Crystallography Laboratory, University of Kansas, Del Shankel Structural Biology Center, 2034 Becker Drive, Lawrence, Kansas 66047

\section{Supporting Information}

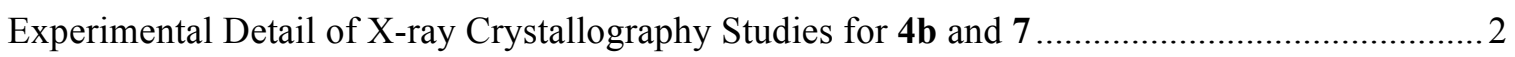

Table S1. Crystallographic data collection and structure refinement for $\mathbf{4 b}$ and $\mathbf{7}$...................... 3

Figure S1. Thermal Ellipsoid Plot of $\mathbf{4 b}$ (50\% probability) …............................................... 4

Figure S2. Thermal Ellipsoid Plot of 7 (50\% probability) .......................................................... 5

${ }^{1} \mathrm{H}$ and ${ }^{13} \mathrm{C}$ NMR Spectra of New Compounds and Known Compounds Prepared with the Present

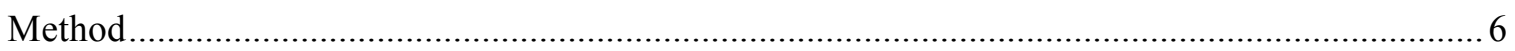




\section{Experimental Detail of X-ray Crystallography Studies for $\mathbf{4 b}$ and 7}

Sets of unique diffraction data $\left[8327(\mathbf{4 b})\right.$ and $7865(7) 0.5^{\circ}$-wide $\omega$ - or $\phi$-scan frames with scan times of 4-15 seconds] were collected ${ }^{[\mathrm{S} 1]}$ at $100(2) \mathrm{K}$ for single-domain crystals of $\mathbf{4 b}$ and 7 using monochromated $\mathrm{CuK} \alpha$ radiation $(\lambda=1.54178 \AA$ ) on a dual-detector Bruker Proteum Single Crystal Diffraction System with a Bruker MicroStar microfocus Cu rotating anode x-ray source operating at $45 \mathrm{kV}$ and $60 \mathrm{~mA}$. Data for $\mathbf{4 b}$ were collected using an APEX II CCD detector equipped with Helios multilayer optics and data for 7 were collected using a Platinum 135 CCD detector equipped with Helios high-brilliance multilayer optics. The integrated data ${ }^{[\mathrm{S} 2]}$ were corrected empirically for variable absorption effects using equivalent reflections. The Bruker software package SHELXTL was used to solve both structures using "direct methods" techniques. All stages of weighted full-matrix least-squares refinement were conducted using $\mathrm{F}_{\mathrm{o}}{ }^{2}$ data with the SHELXTL XL program in the Bruker APEX2 v2014.11-0 software package ${ }^{[\mathrm{S3}]}$. Final crystallographic details are summarized in Table S1.

The final structural models for both structures incorporated anisotropic thermal parameters for all nonhydrogen atoms except carbon atom C3' of 7 which was modeled with an isotropic thermal parameter. Isotropic thermal parameters were incorporated for all hydrogen atoms of both structures.

The asymmetric unit of $\mathbf{4 b}$ has two $55 / 45$ disordered $\mathrm{C}_{12} \mathrm{H}_{15} \mathrm{~N}_{5}$ molecules with slightly different orientations in the lattice. The nonhydrogen atoms in these two molecules were restrained to have similar bond lengths and angles. Mild restraints were applied to the anisotropic thermal parameters of $\mathrm{N} 4, \mathrm{~N} 5 \mathrm{C} 2$ and $\mathrm{C} 6$ '.

The 8-atom C2-C9 phenyl-containing fragment for the $\mathrm{C}_{15} \mathrm{H}_{14} \mathrm{~N}_{2} \mathrm{O}_{2}$ molecule in the asymmetric unit of 7 is $51 / 49$ disordered with two slightly different orientations. The phenyl rings for both disordered groupings were incorporated into the structural model as a hexagonal rigid body with $\mathrm{C}-\mathrm{C}$ bond lengths of $1.39 \AA$. The nitrogen $(\mathrm{H} 1 \mathrm{~N})$ and oxygen $(\mathrm{H} 2 \mathrm{O})$ hydrogen atoms for 7 as well as those on the ordered heterocycle were located in a difference Fourier and initially included in the structural model as independent isotropic atoms whose parameters were allowed to vary in least-squares refinement cycles. Even though these six hydrogen atoms all refined satisfactorily, only the positional parameters for $\mathrm{H} 1 \mathrm{~N}, \mathrm{H} 2 \mathrm{O}$ and $\mathrm{H} 11$ were allowed to vary in the final least-squares cycles in order to reduce the number of variables. The isotropic thermal parameters for $\mathrm{H} 1 \mathrm{~N}$ and $\mathrm{H} 2 \mathrm{O}$ were also allowed to vary.

The remaining hydrogen atoms for the both structures were included in the structural models as idealized atoms (assuming $\mathrm{sp}^{2}$-hybridization of the nitrogen and N-H bond lengths of $0.88 \AA$ in $\mathbf{4} \mathbf{b}$ and $\mathrm{sp}^{2}$ - or $\mathrm{sp}^{3}$-hybridization of the carbon and $\mathrm{C}-\mathrm{H}$ bond lengths of $0.95-1.00 \AA$ in 4b and 7). The isotropic thermal parameters of all idealized hydrogen atoms in both structures and H11 in 7 were fixed at values 1.2 (nonmethyl) or 1.5 (methyl) times the equivalent isotropic thermal parameter of the carbon or nitrogen atom to which they are covalently bonded. 
Table S1. Crystallographic data collection and structure refinement for $\mathbf{4 b}$ and $\mathbf{7 .}$

\begin{tabular}{|c|c|c|}
\hline Compound & $4 \mathrm{~b}$ & 7 \\
\hline Formula & $\mathrm{C}_{12} \mathrm{H}_{15} \mathrm{~N}_{5}$ & $\mathrm{C}_{15} \mathrm{H}_{14} \mathrm{~N}_{2} \mathrm{O}_{2}$ \\
\hline Formula weight & 229.29 & 254.28 \\
\hline Crystal system & Monoclinic & Monoclinic \\
\hline Space group & $\mathrm{P} 2_{1} / \mathrm{c}($ No. 14$)$ & $\mathrm{P} 2_{1} / \mathrm{c}($ No. 14$)$ \\
\hline$a(\AA)$ & $5.9910(6)$ & $12.8994(7)$ \\
\hline$b(\AA)$ & $28.946(3)$ & $10.6296(5)$ \\
\hline$c(\AA)$ & $7.1474(7)$ & $9.5520(5)$ \\
\hline$\alpha\left(^{\circ}\right)$ & 90.000 & 90.000 \\
\hline$\beta\left(^{\circ}\right)$ & $111.950(6)$ & $99.323(1)$ \\
\hline$\gamma\left({ }^{\circ}\right)$ & 90.000 & 90.000 \\
\hline$V\left(\AA^{3}\right)$ & $1149.6(2)$ & $1292.4(1)$ \\
\hline$Z$ & 4 & 4 \\
\hline$\rho_{\text {calcd }}\left(\mathrm{g} \mathrm{cm}^{-3}\right)$ & 1.325 & 1.307 \\
\hline$\lambda(\AA)$ & 1.54178 & 1.54178 \\
\hline$T(\mathrm{~K})$ & $100(2)$ & $100(2)$ \\
\hline$F(000)$ & 488 & 536 \\
\hline Crystal size $\left(\mathrm{mm}^{3}\right)$ & $0.09 \times 0.17 \times 0.28$ & $0.05 \times 0.08 \times 0.11$ \\
\hline$\mu\left(\mathrm{mm}^{-1}\right)$ & 0.68 & 0.715 \\
\hline Abs corr & Multi-scan & Multi-scan \\
\hline Max, min trans & $1.000,0.753$ & $1.000,0.857$ \\
\hline$\theta$ range $\left(^{\circ}\right)$ & 3.05 to 69.84 & 5.42 to 68.04 \\
\hline Completeness to theta $=66.00^{\circ}$ & $96.2 \%$ & $98.3 \%$ \\
\hline Reflns collected & 8327 & 7865 \\
\hline Indep reflns & 2031 & 2262 \\
\hline$R($ int $)$ & 0.035 & 0.018 \\
\hline Data/restr/param & $2031 / 68 / 309$ & $2262 / 0 / 228$ \\
\hline${ }^{a} R_{1} ; \mathrm{w} R_{2}[\mathrm{I}>2 \sigma(\mathrm{I})]$ & $0.065 ; 0.190$ & $0.057 ; 0.135$ \\
\hline${ }^{a} R_{1} ; \mathrm{w} R_{2}$ (all data) & $0.070 ; 0.193$ & $0.058 ; 0.136$ \\
\hline $\operatorname{GOF}\left(F^{2}\right)$ & 1.120 & 1.092 \\
\hline $\begin{array}{l}\text { Largest diff. peak } \\
\text { and hole }\left(\mathrm{e}^{-} \AA^{-3}\right)\end{array}$ & $0.28,-0.21$ & $0.34,-0.21$ \\
\hline
\end{tabular}

[S1] Data Collection: SMART Software in APEX2 v2014.11-0 Suite. Bruker-AXS, 5465 E. Cheryl Parkway, Madison, WI 53711-5373 USA.

[S2] Data Reduction: SAINT Software in APEX2 v2014.11-0 Suite. Bruker-AXS, 5465 E. Cheryl Parkway, Madison, WI 53711-5373 USA.

[S3] Refinement: SHELXTL Software in APEX2 v2014.11-0 Suite. Bruker-AXS, 5465 E. Cheryl Parkway, Madison, WI 53711-5373 USA. 
Figure S1. Thermal Ellipsoid Plot of $\mathbf{4 b}$ (50\% probability).

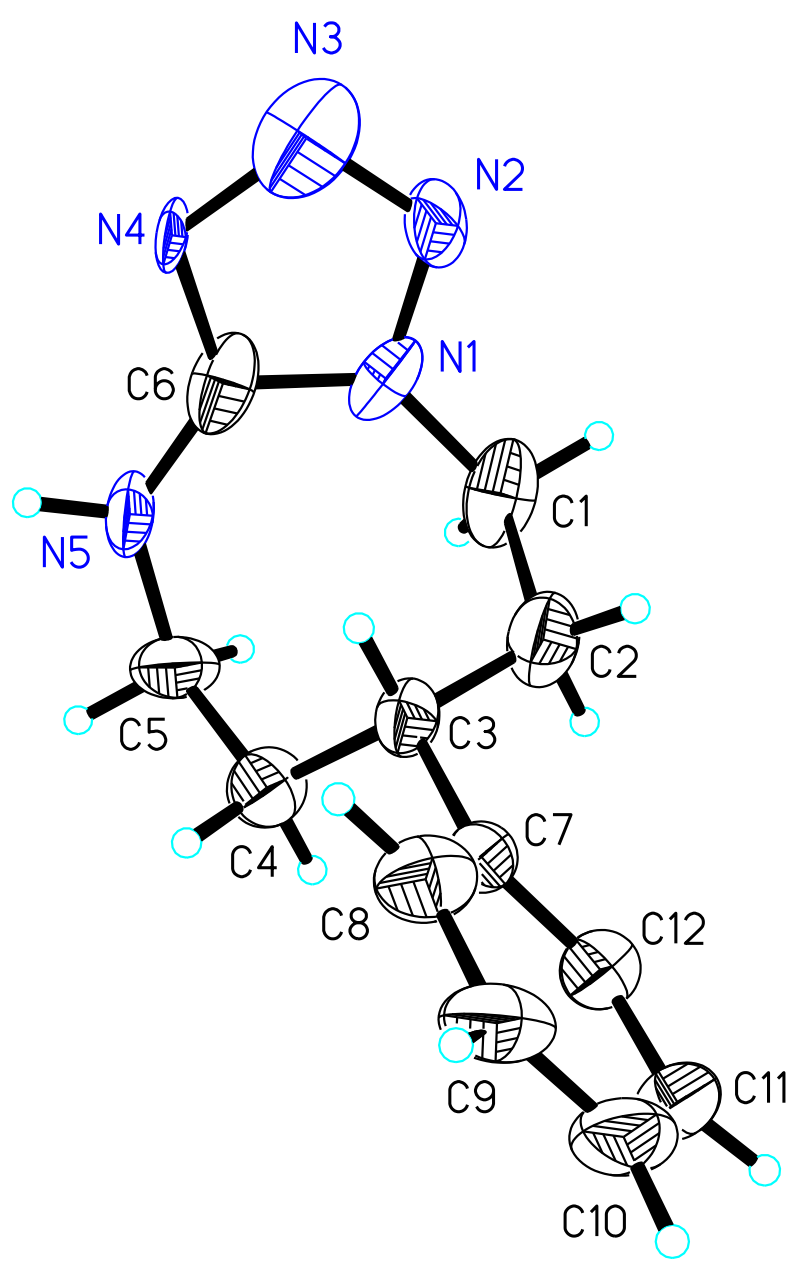


Figure S2. Thermal Ellipsoid Plot of 7 (50\% probability).

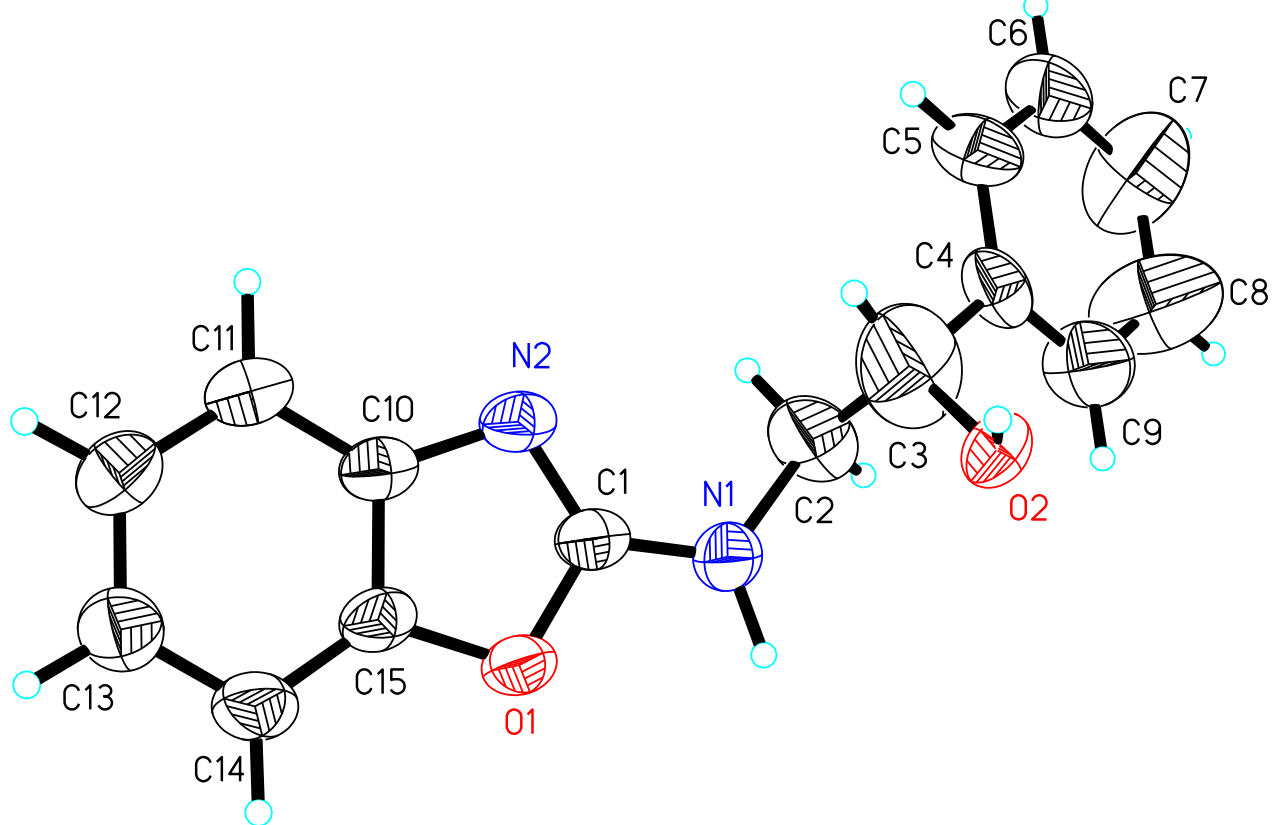


${ }^{1} \mathrm{H}$ and ${ }^{13} \mathrm{C}$ NMR Spectra of New Compounds and Known Compounds Prepared with the Present Method 

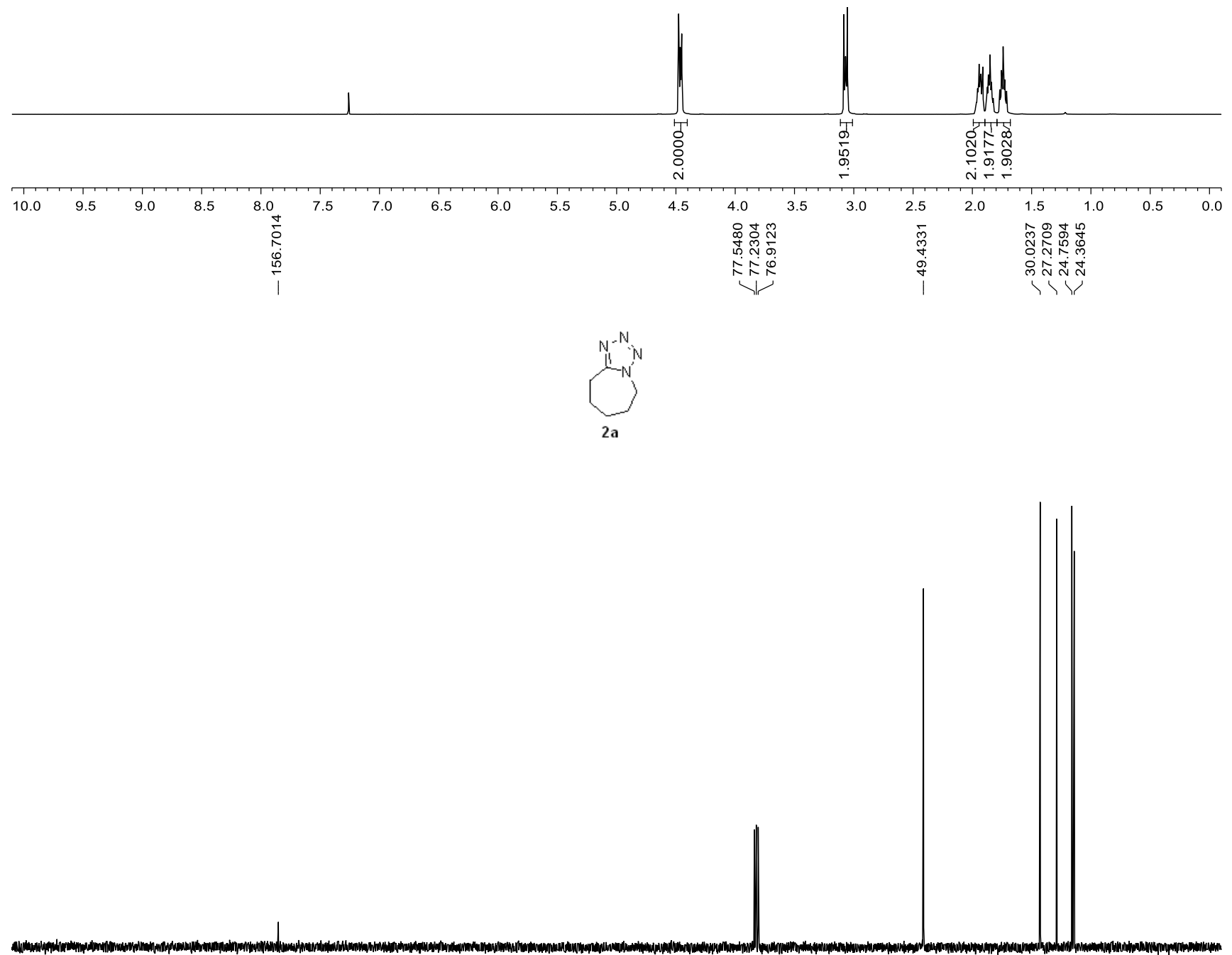

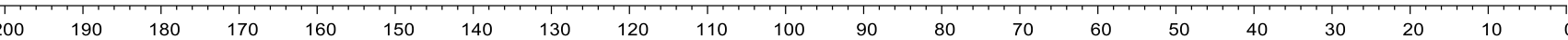



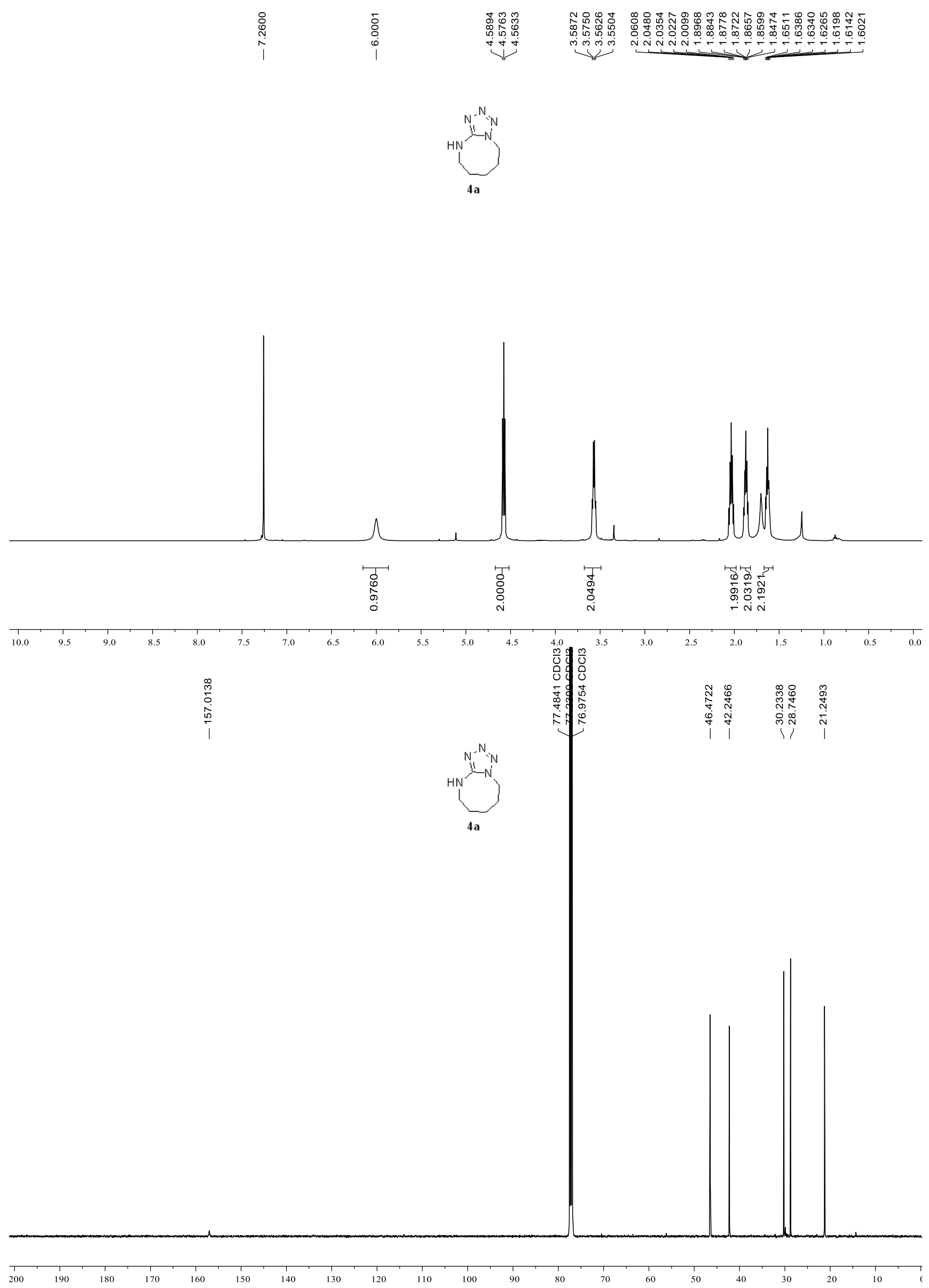

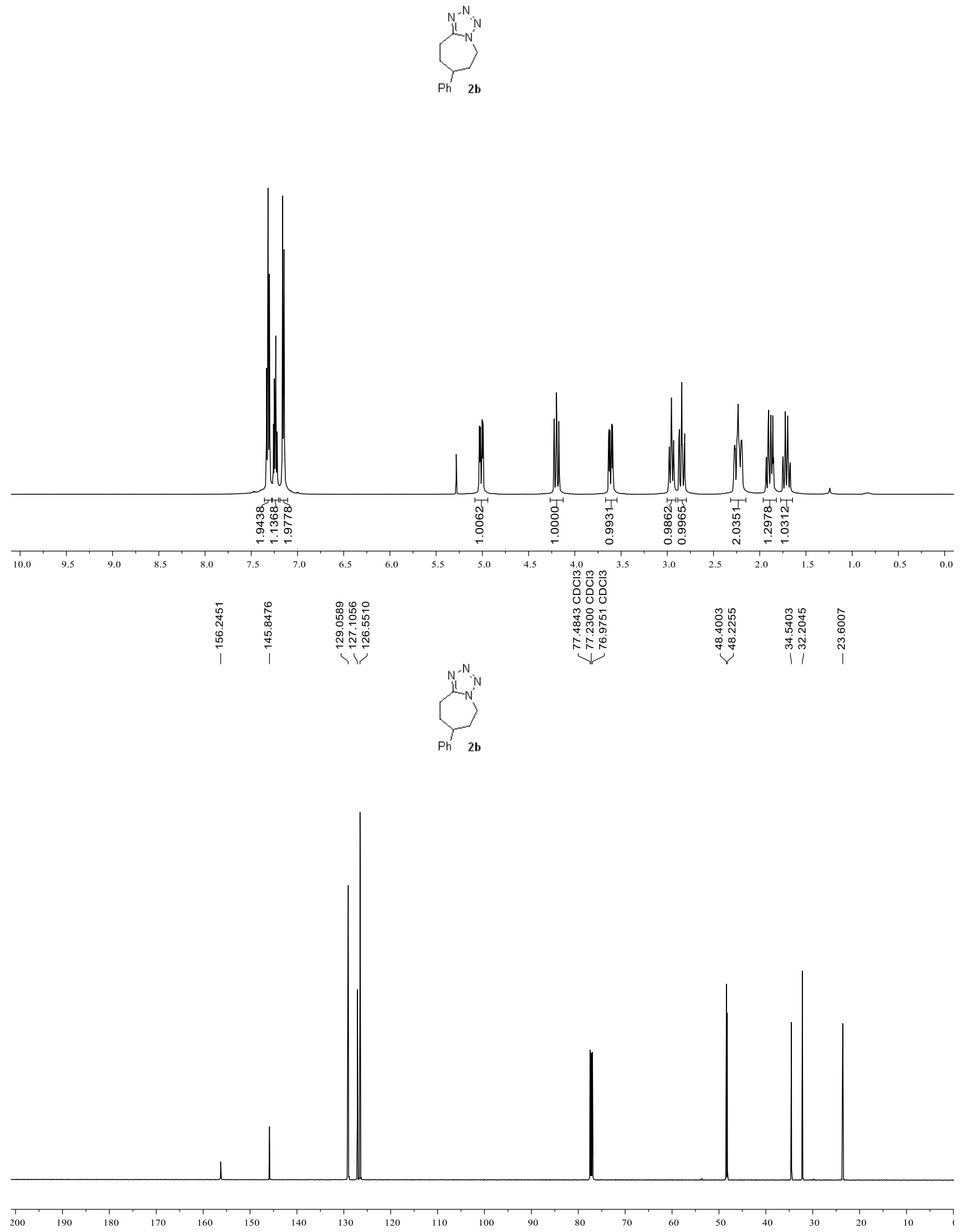

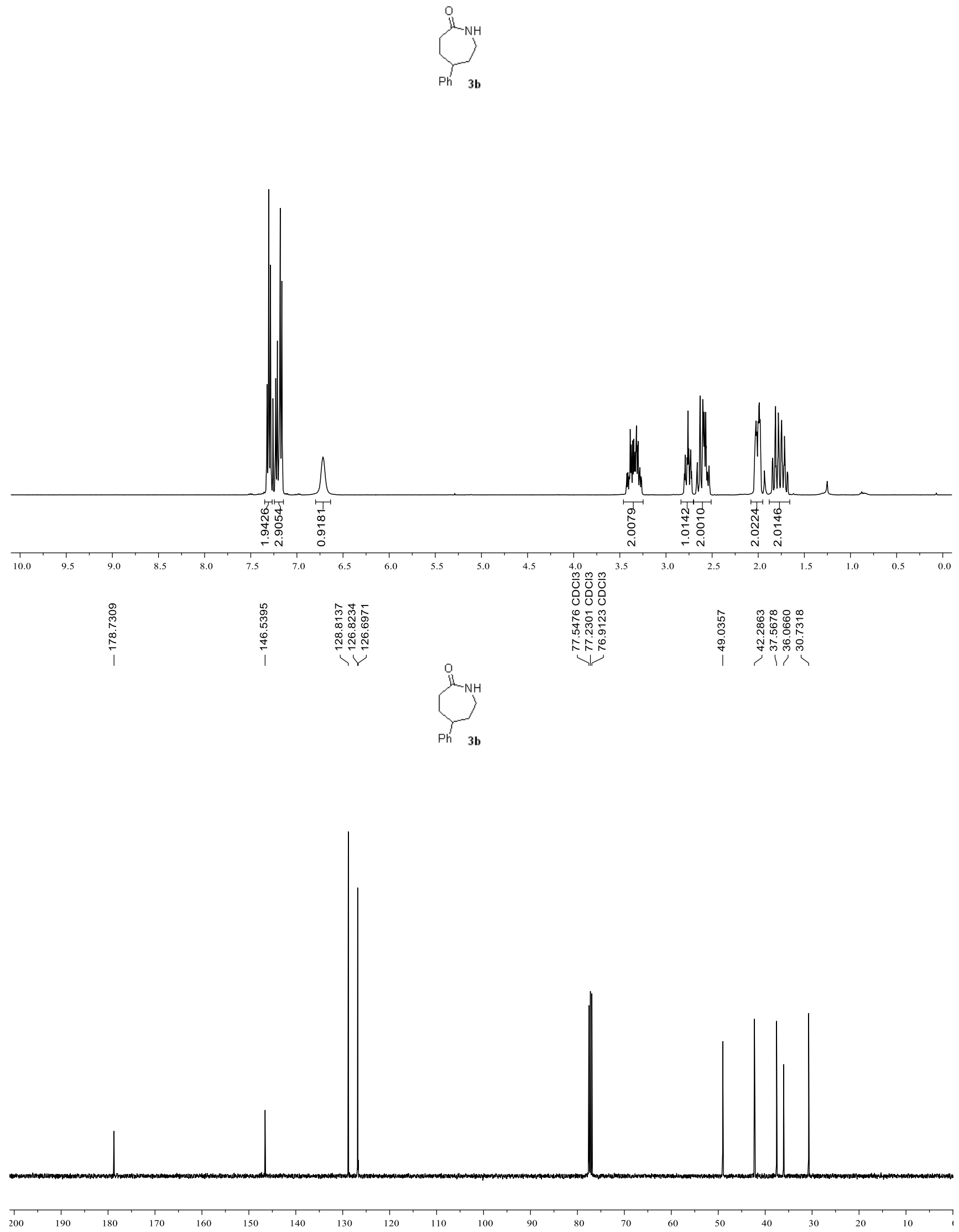

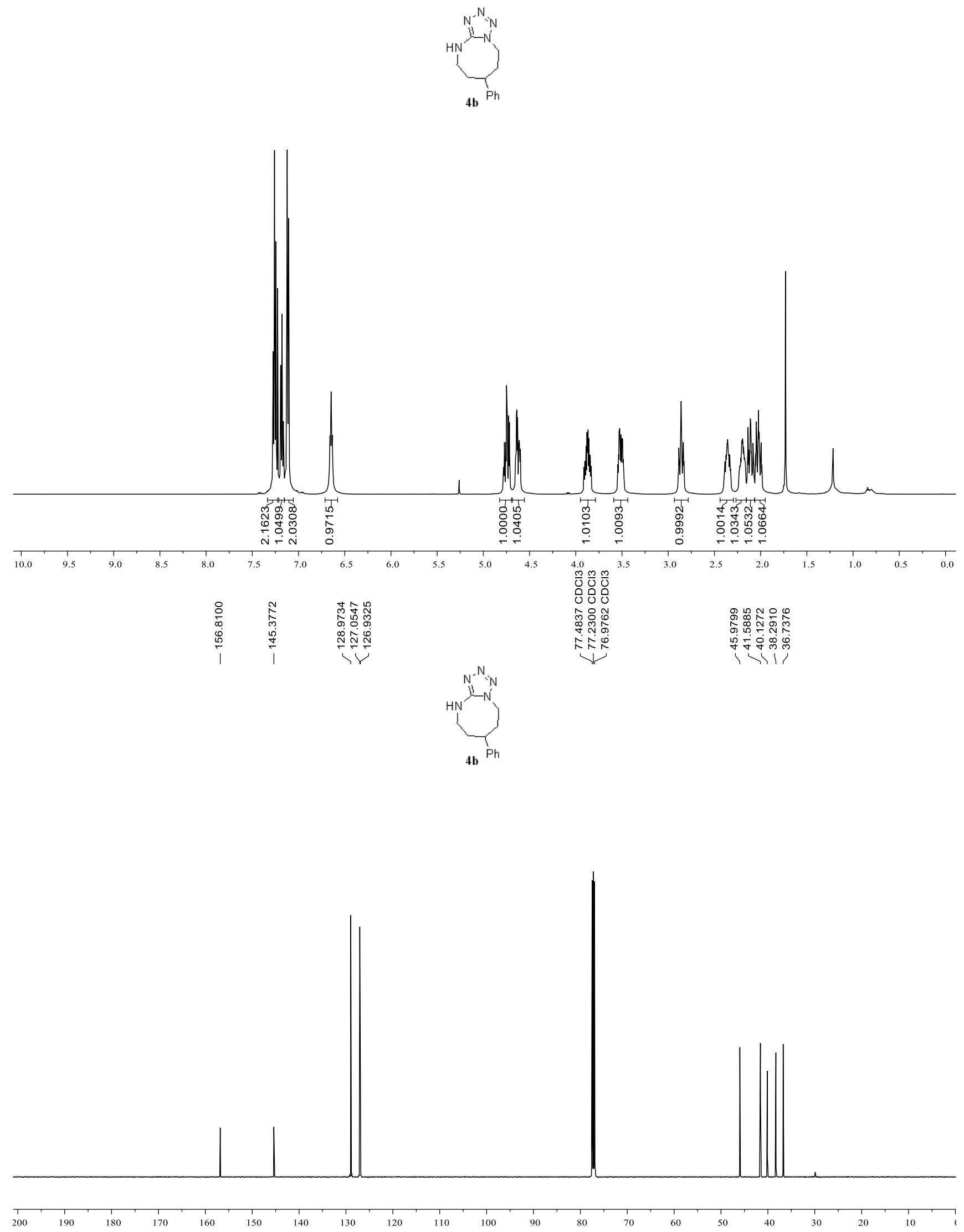

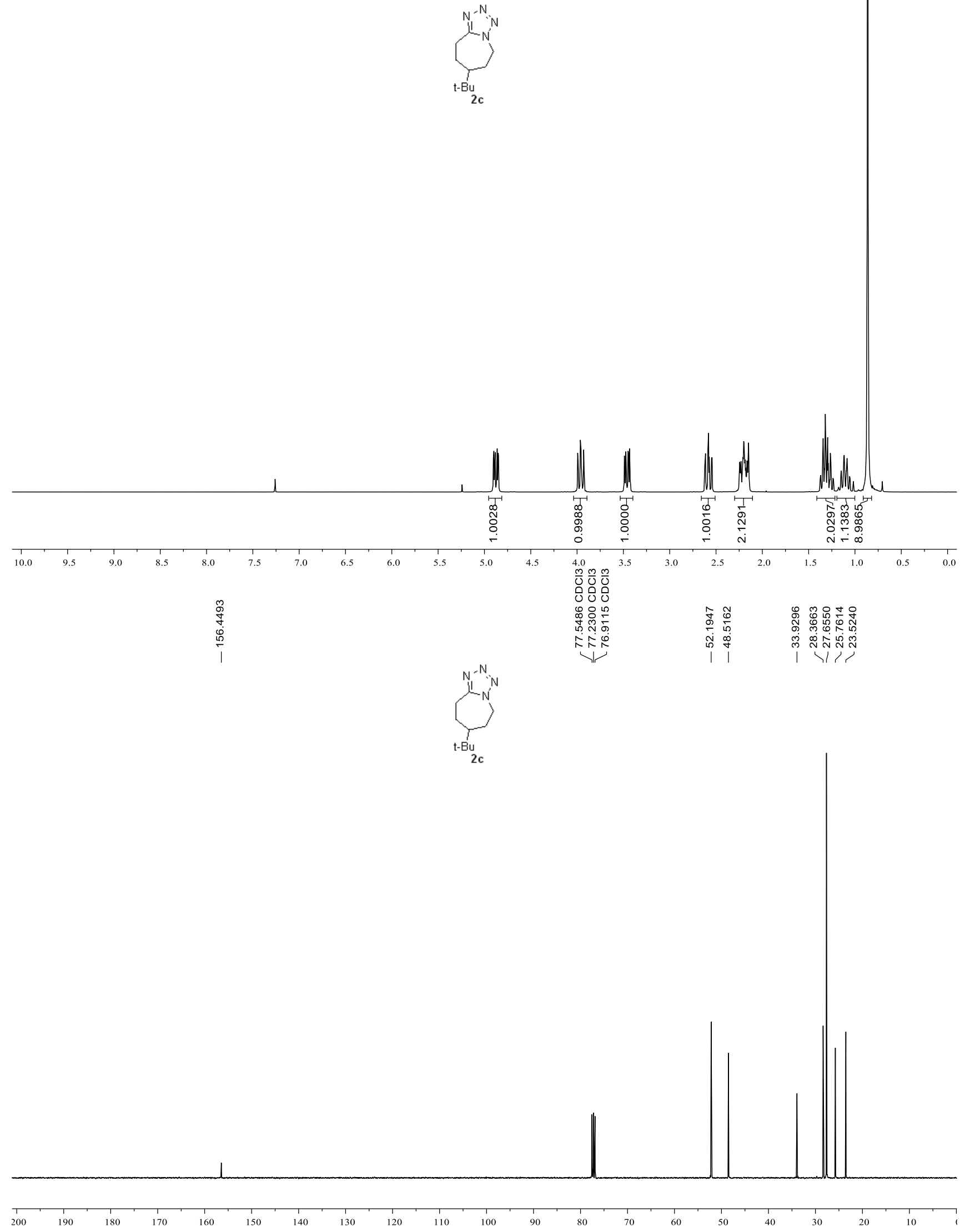

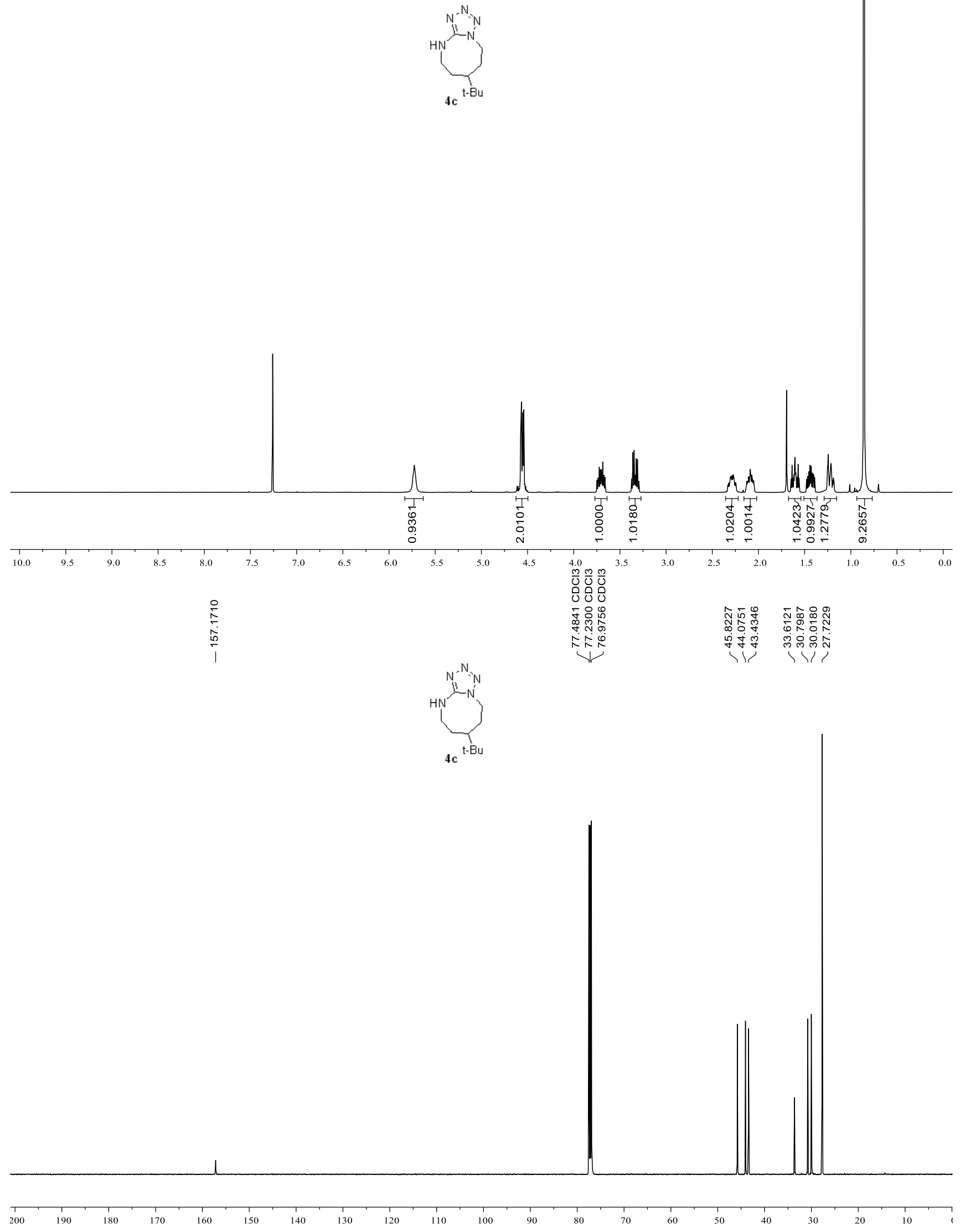

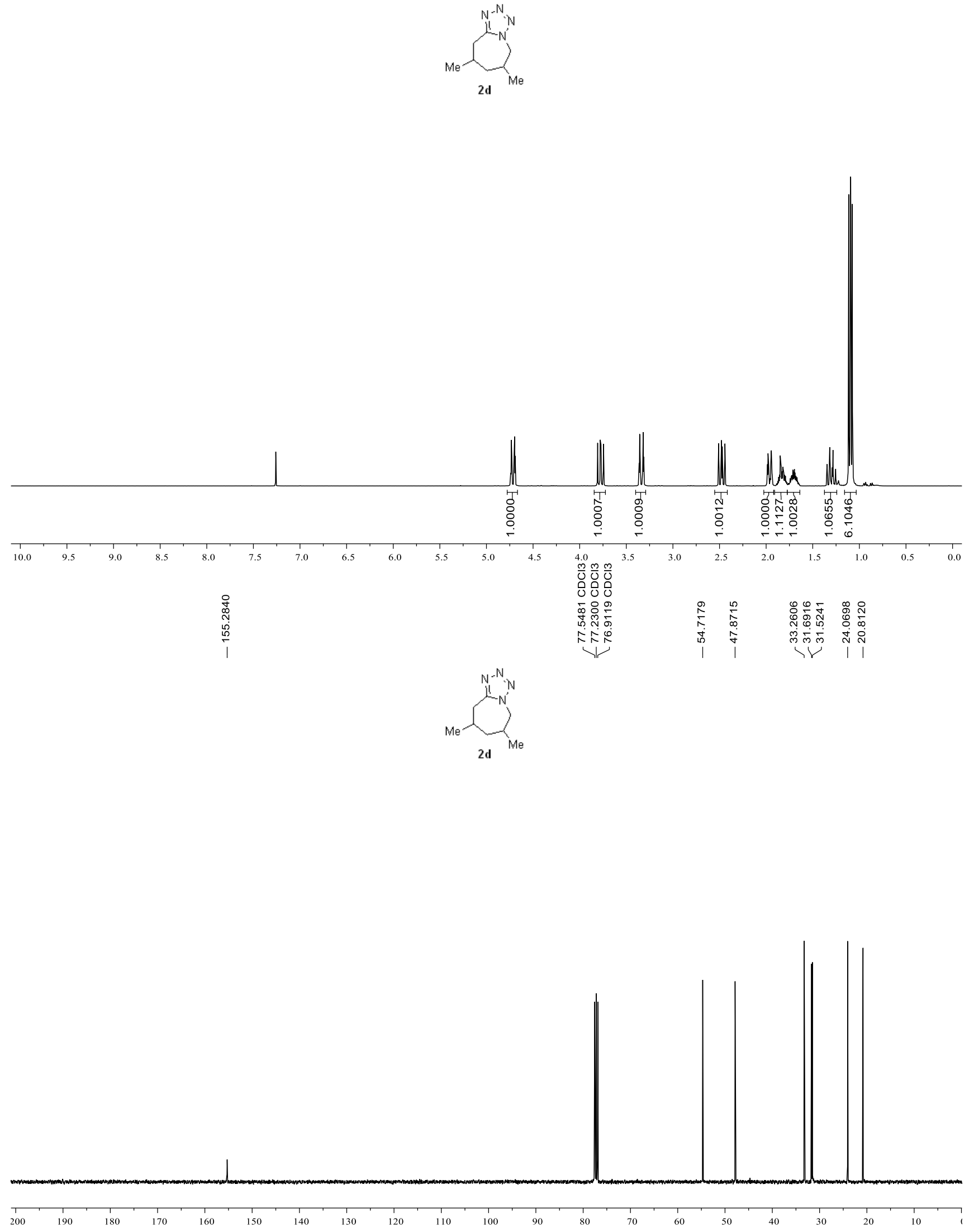


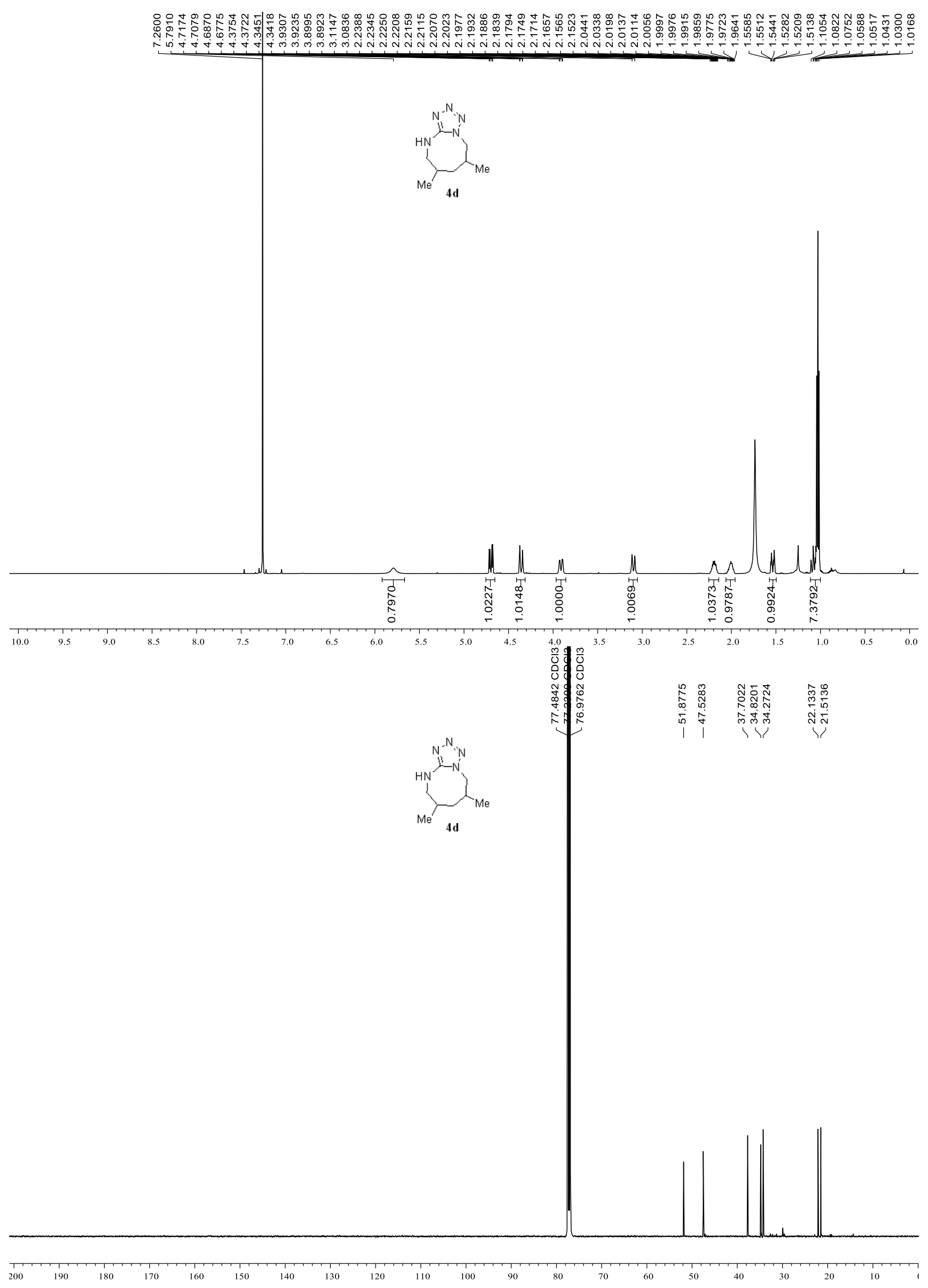


$\sum_{0}^{N}$

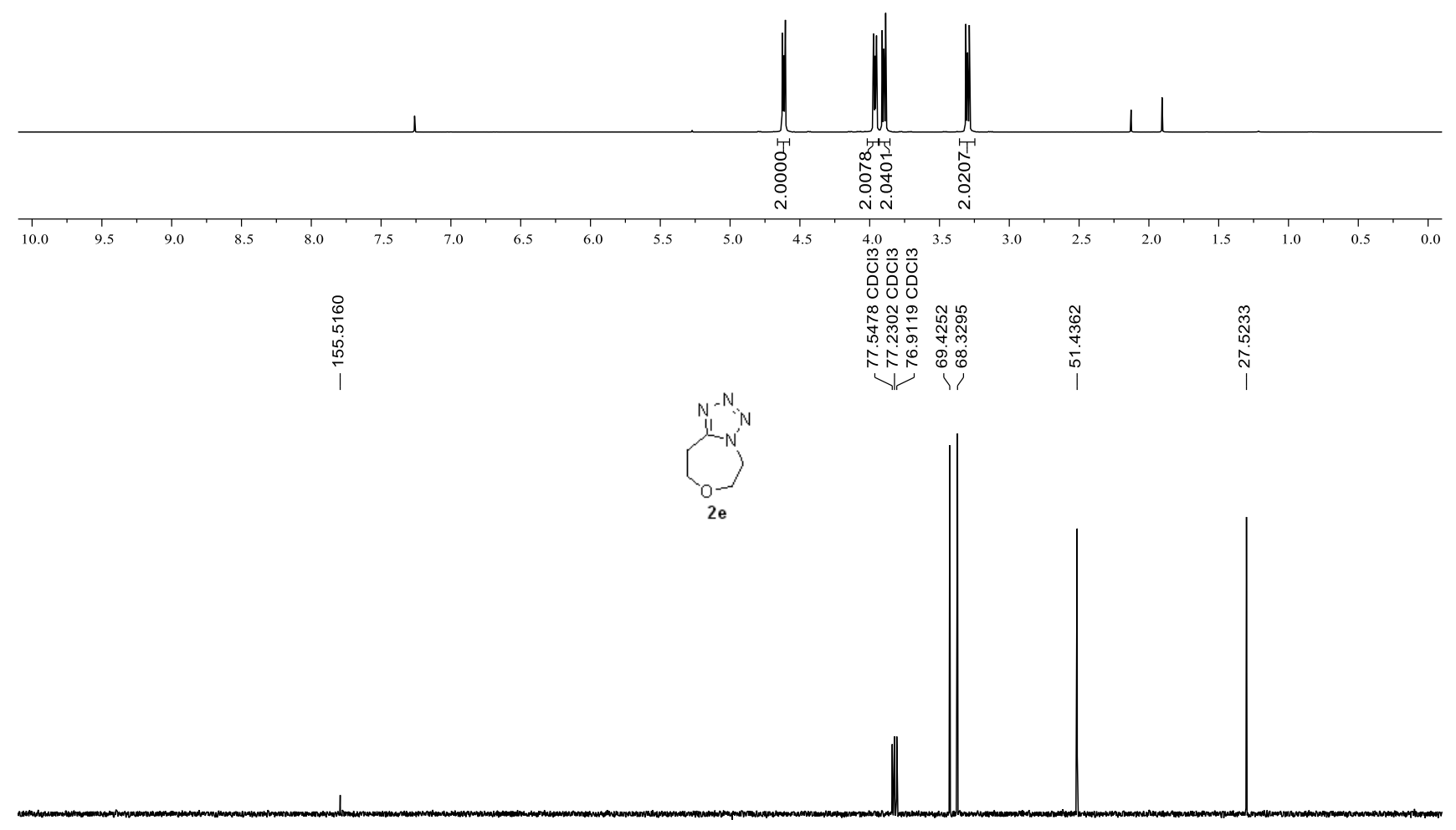

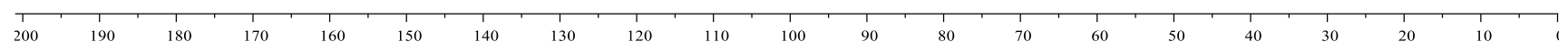




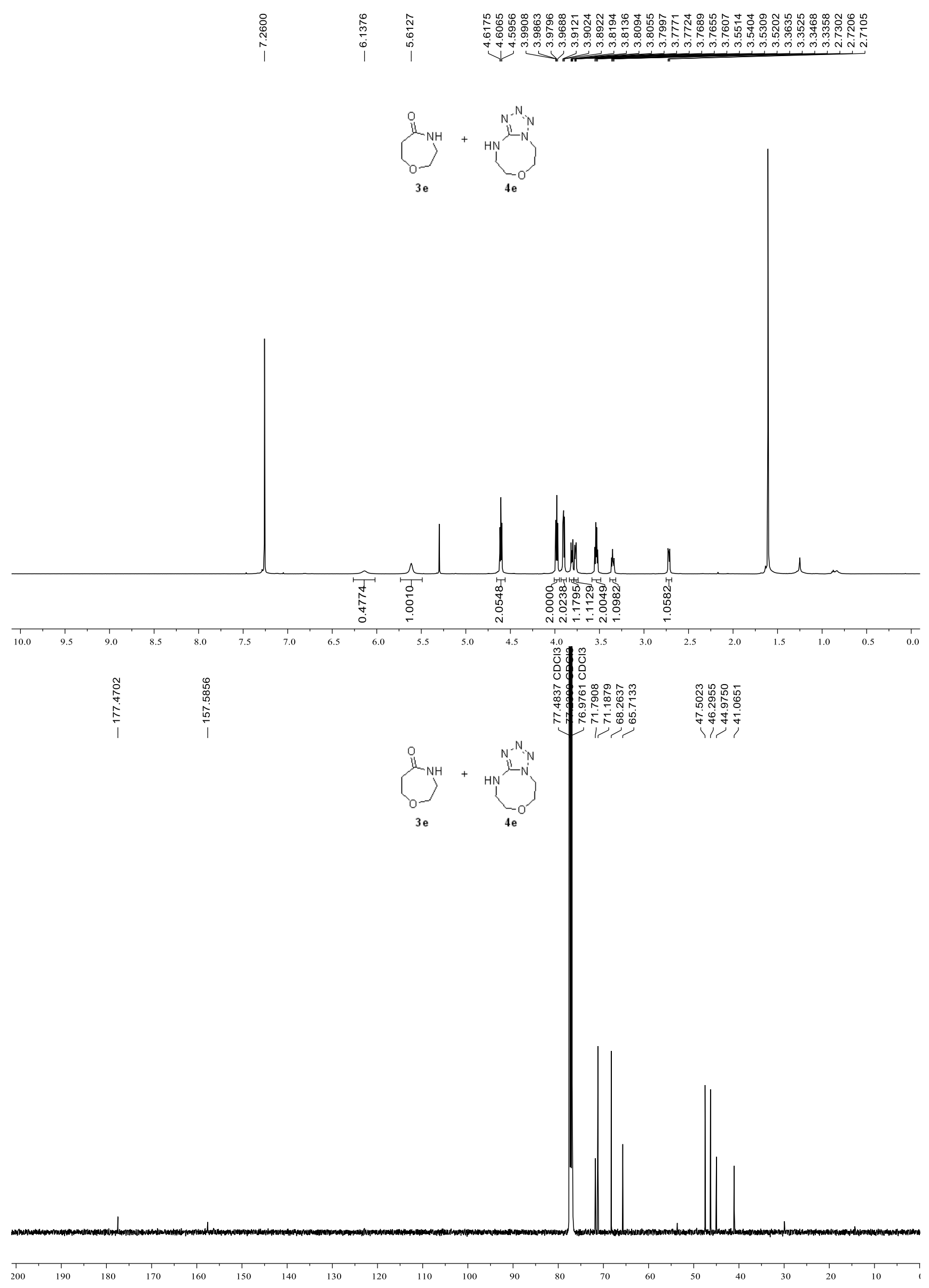



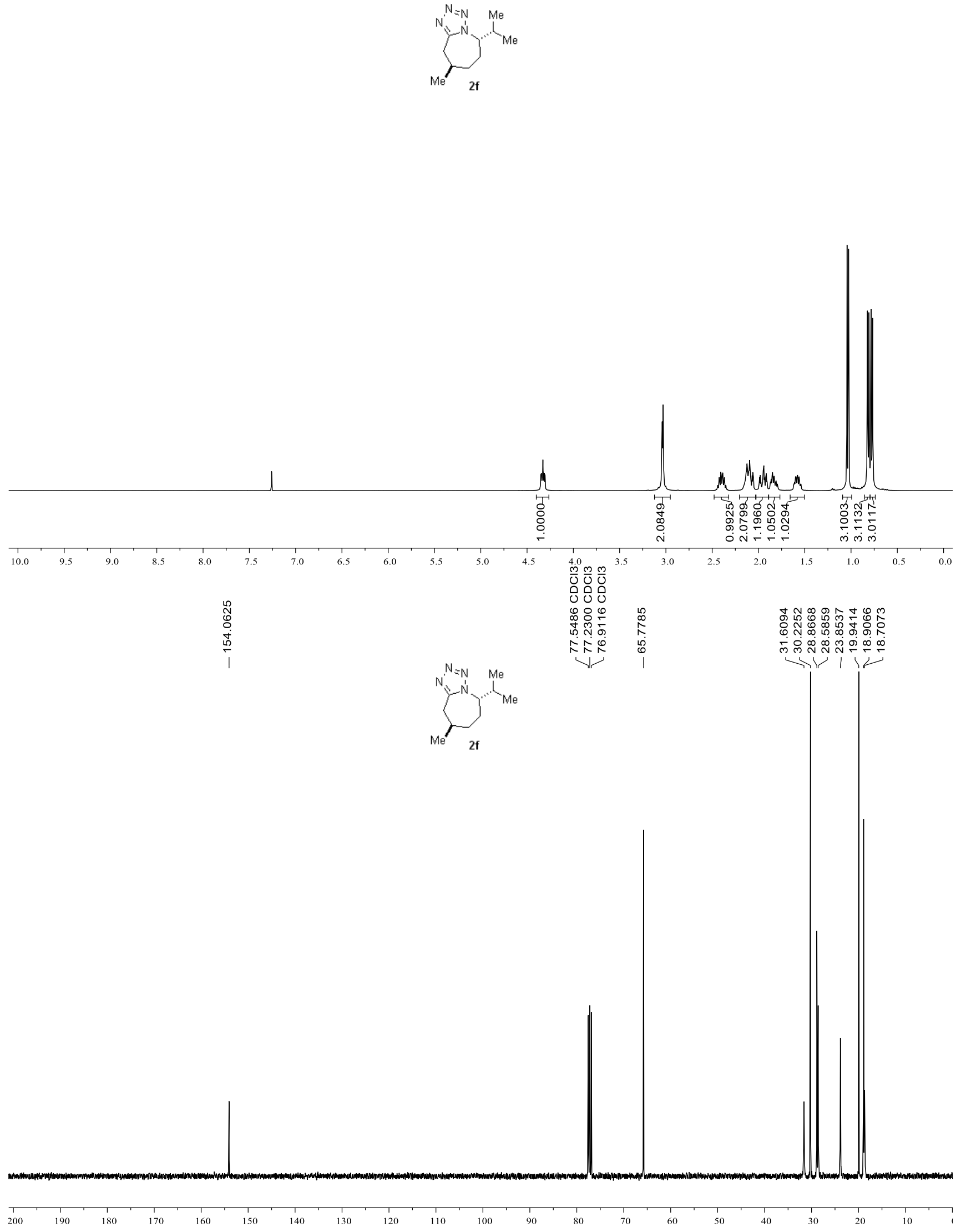


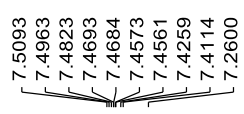

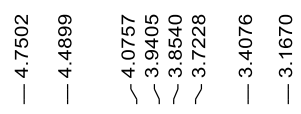
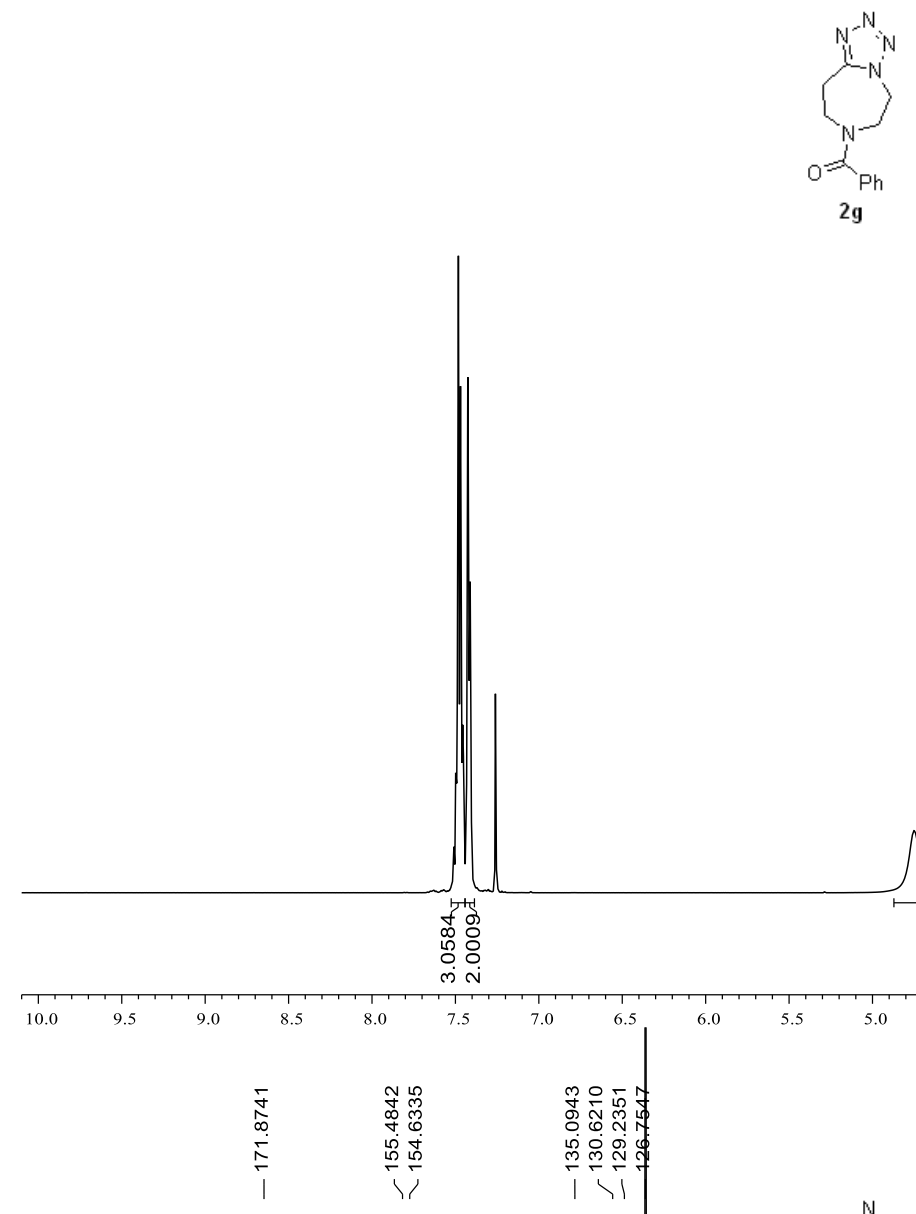


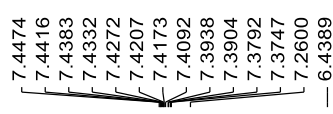

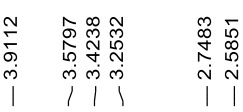
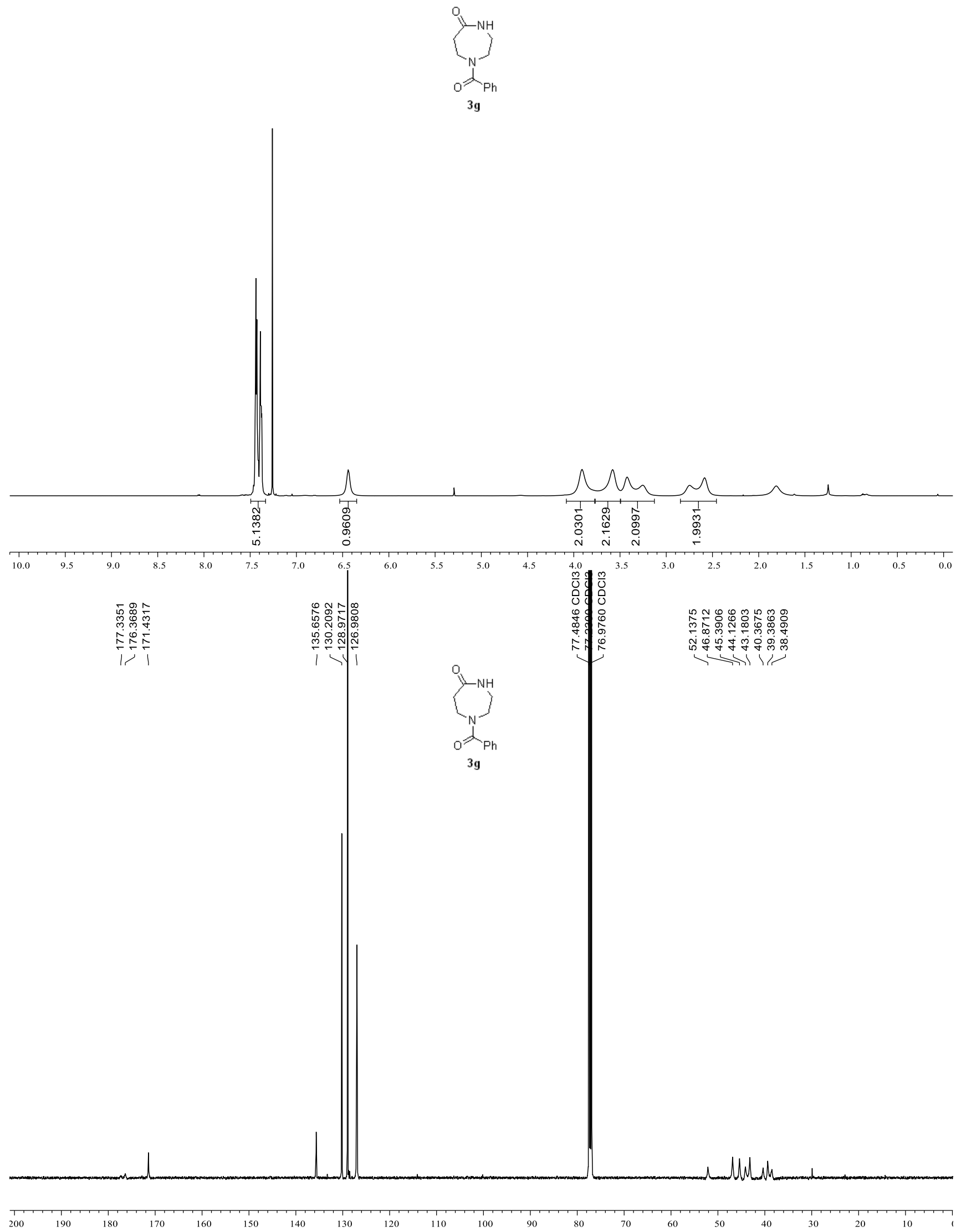

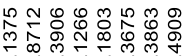

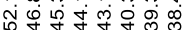

作 

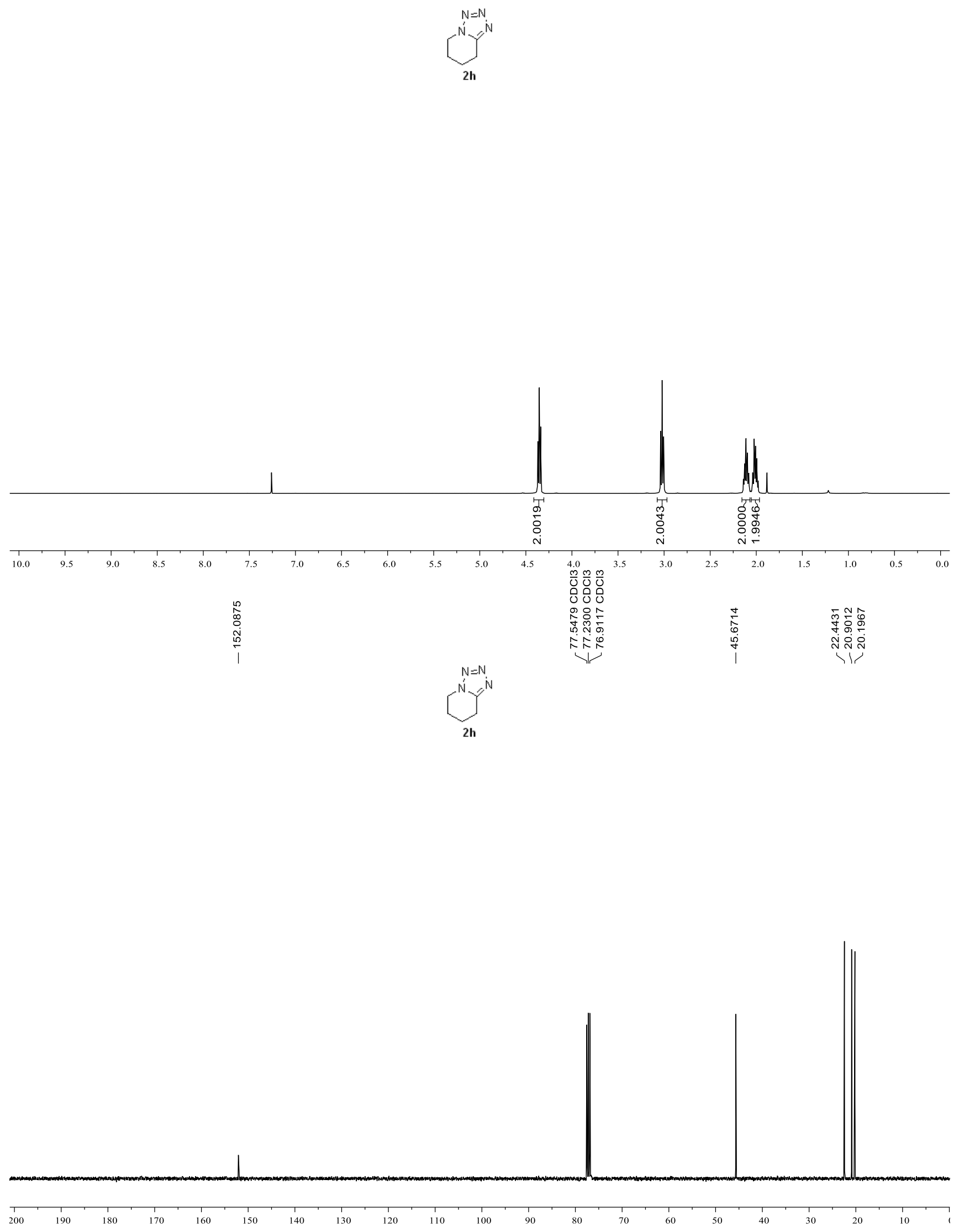

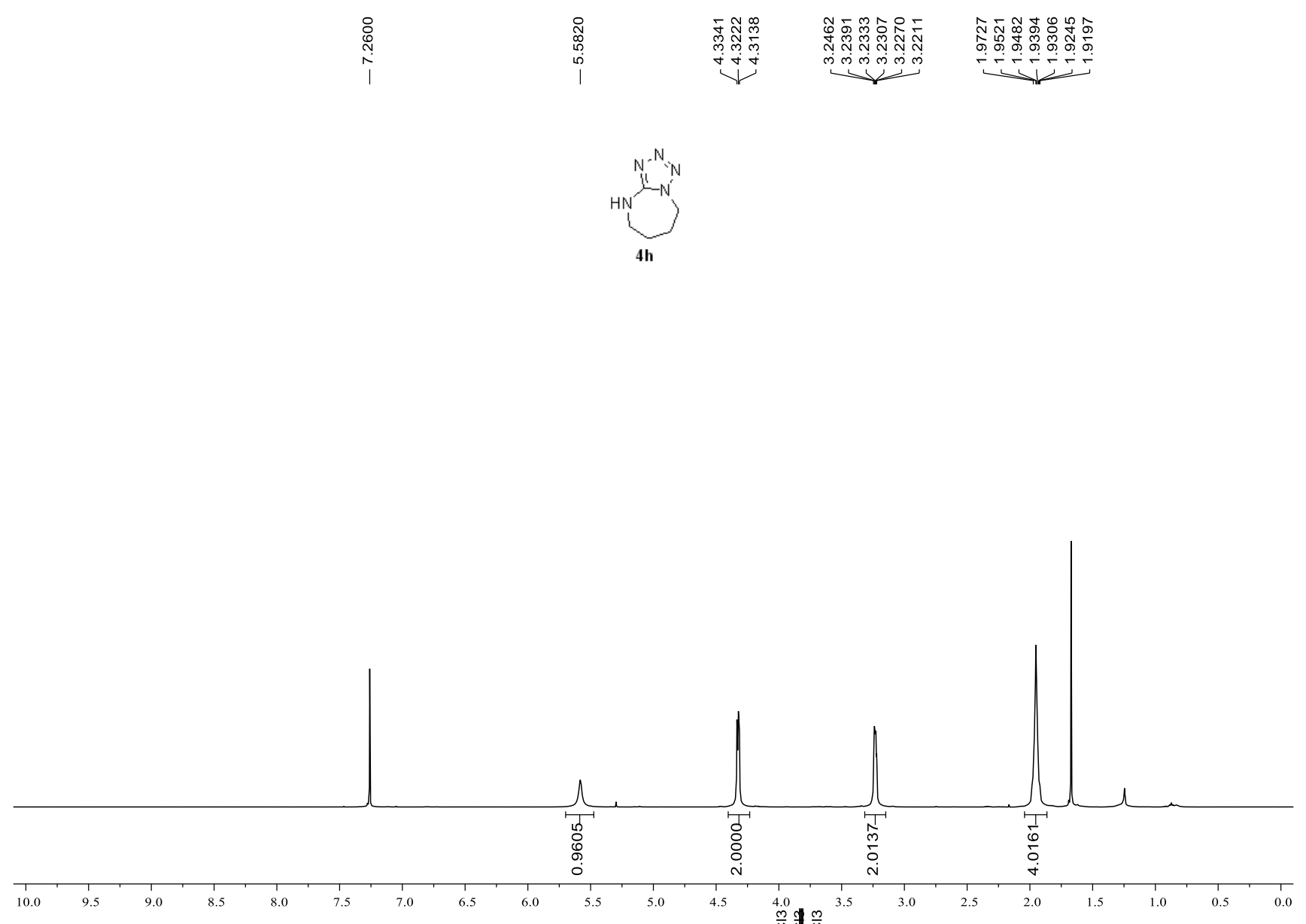

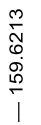
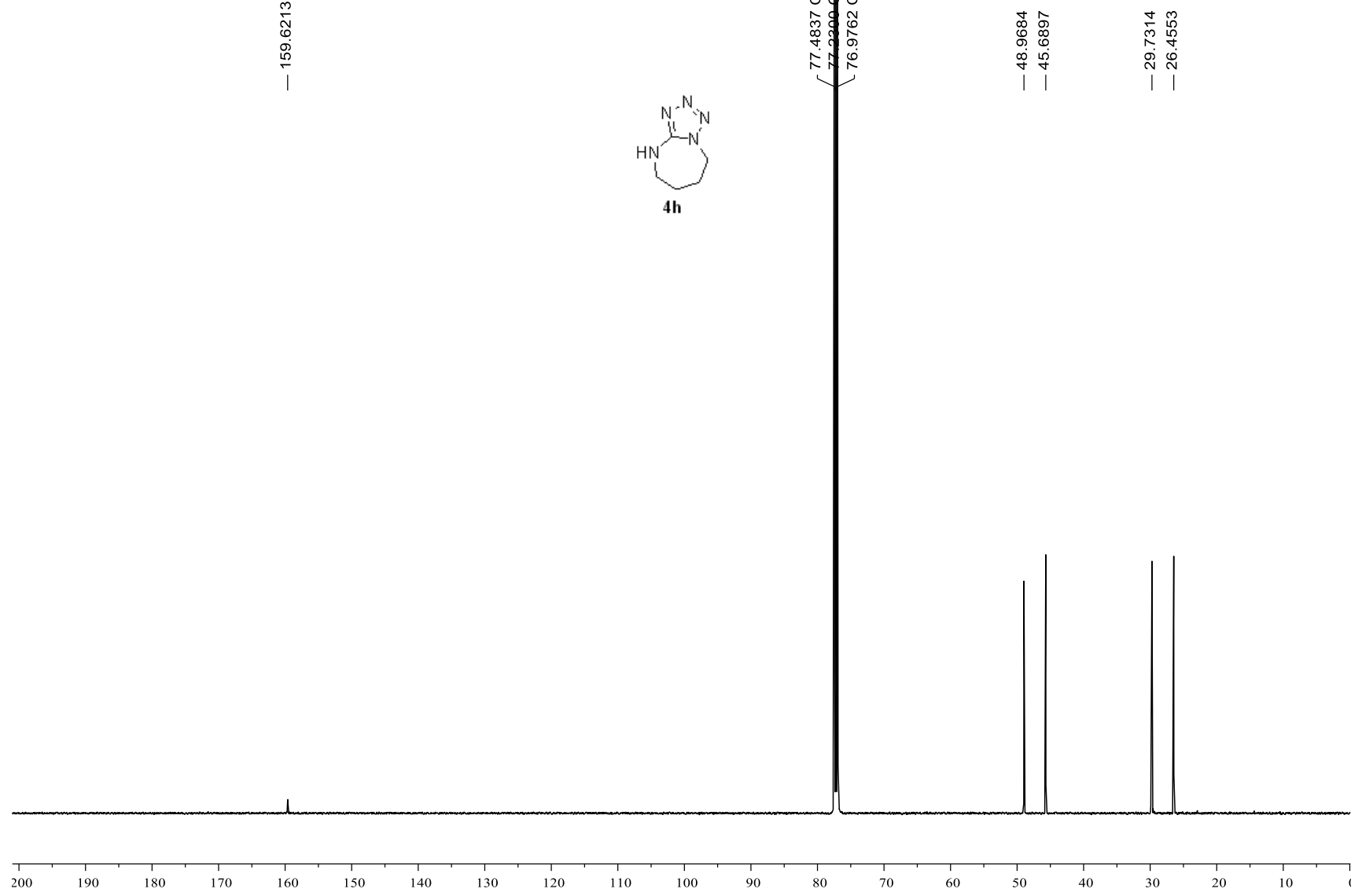

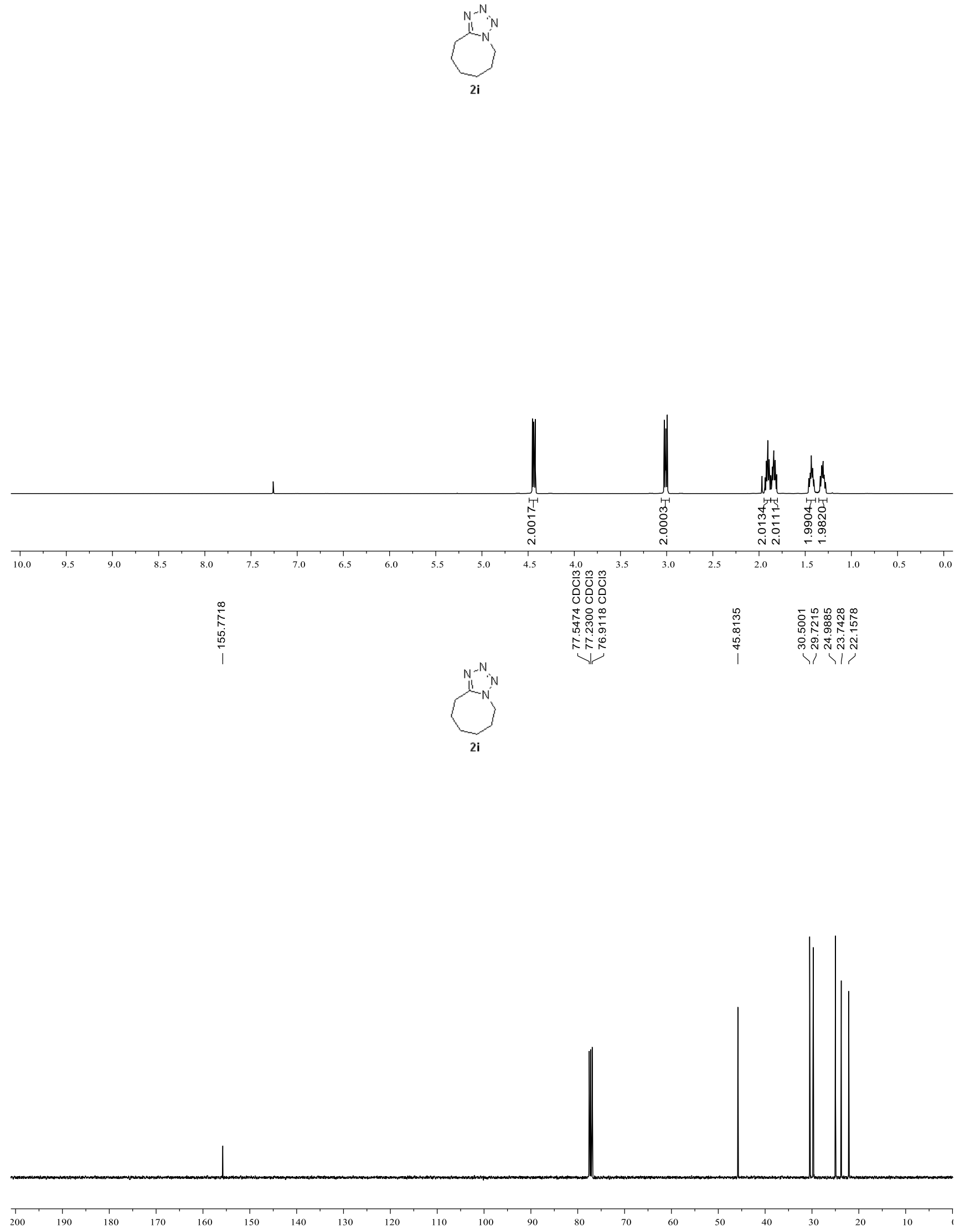

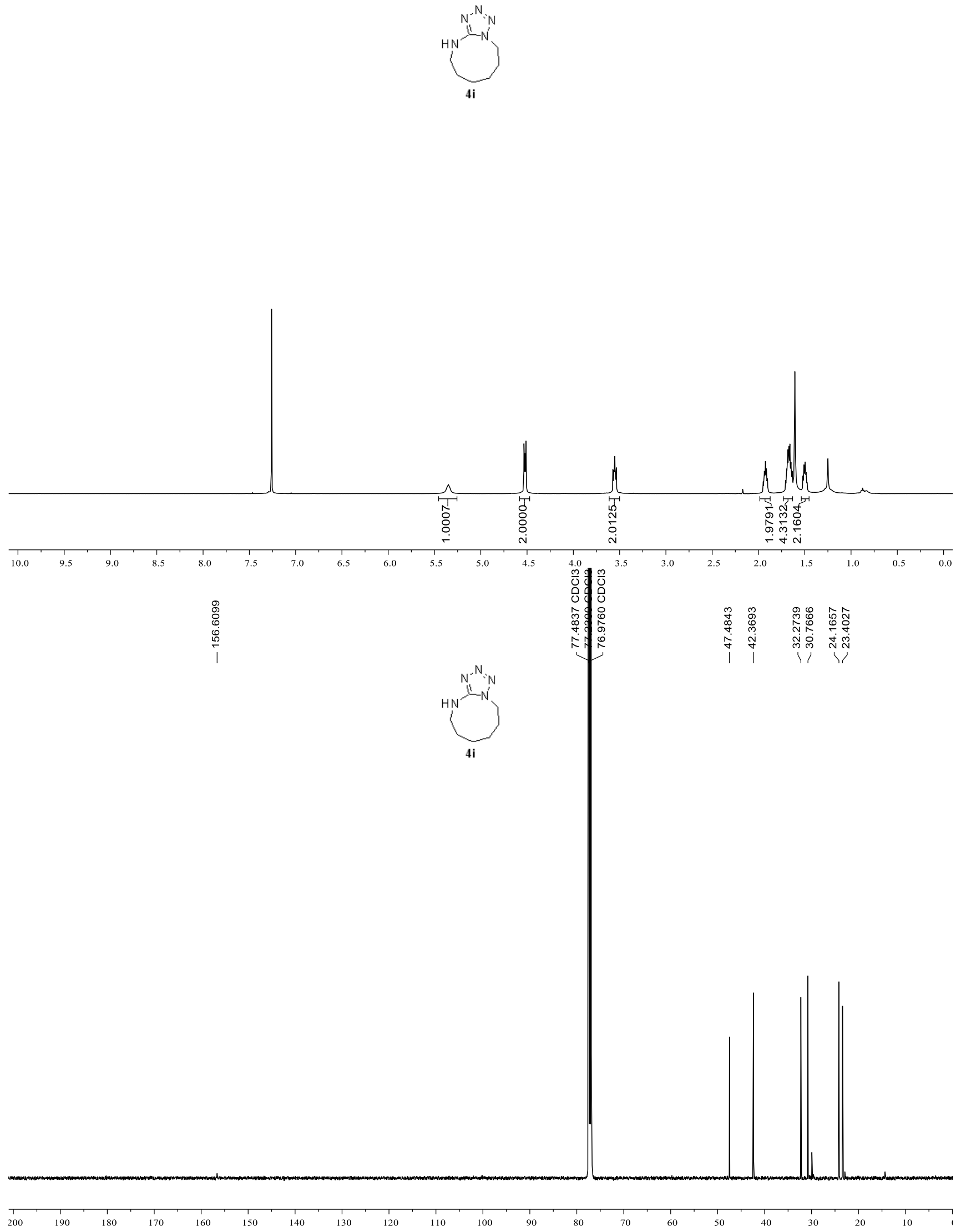

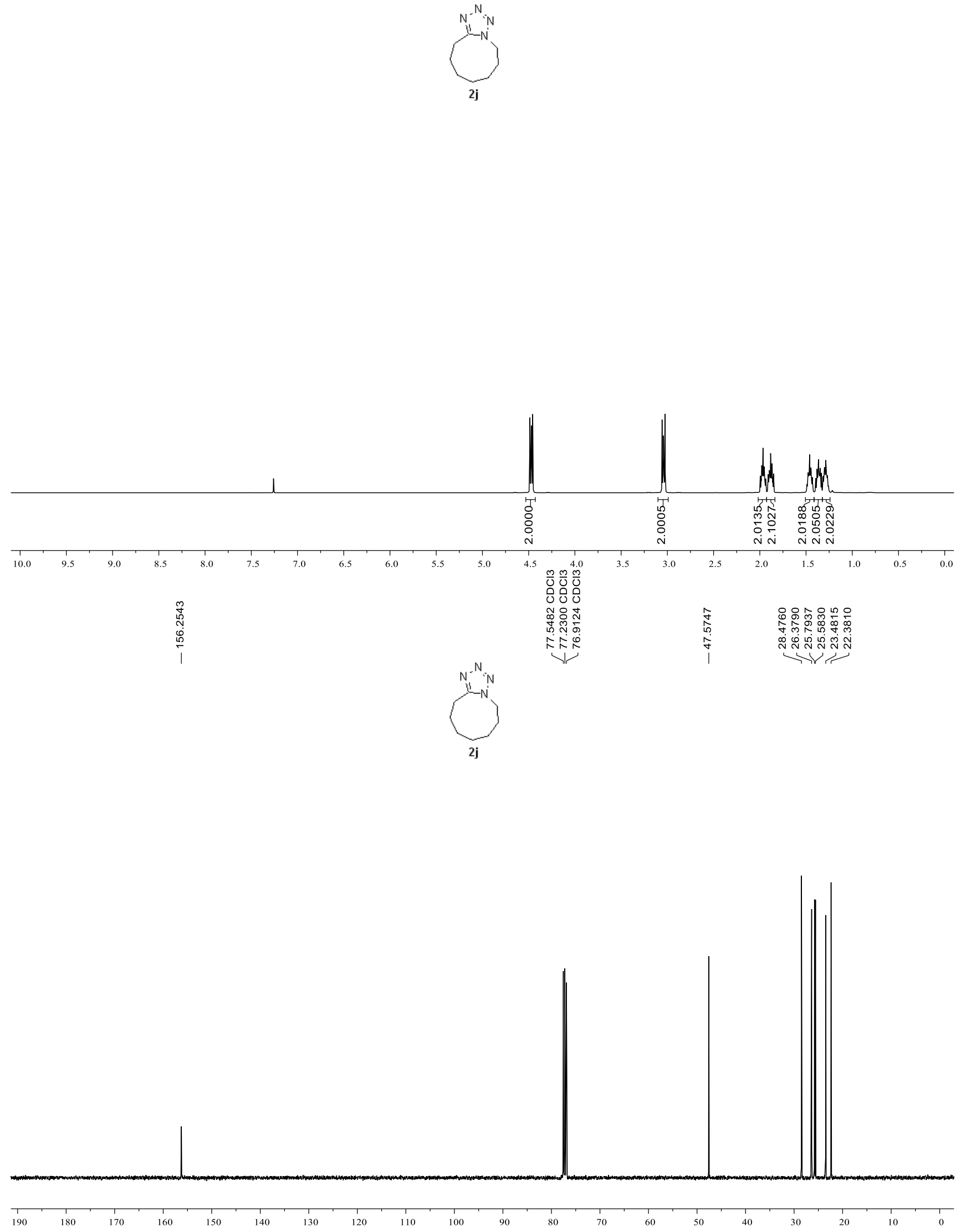


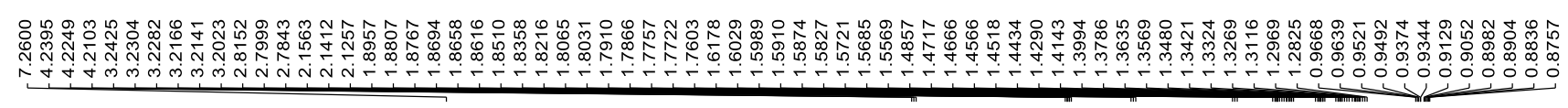
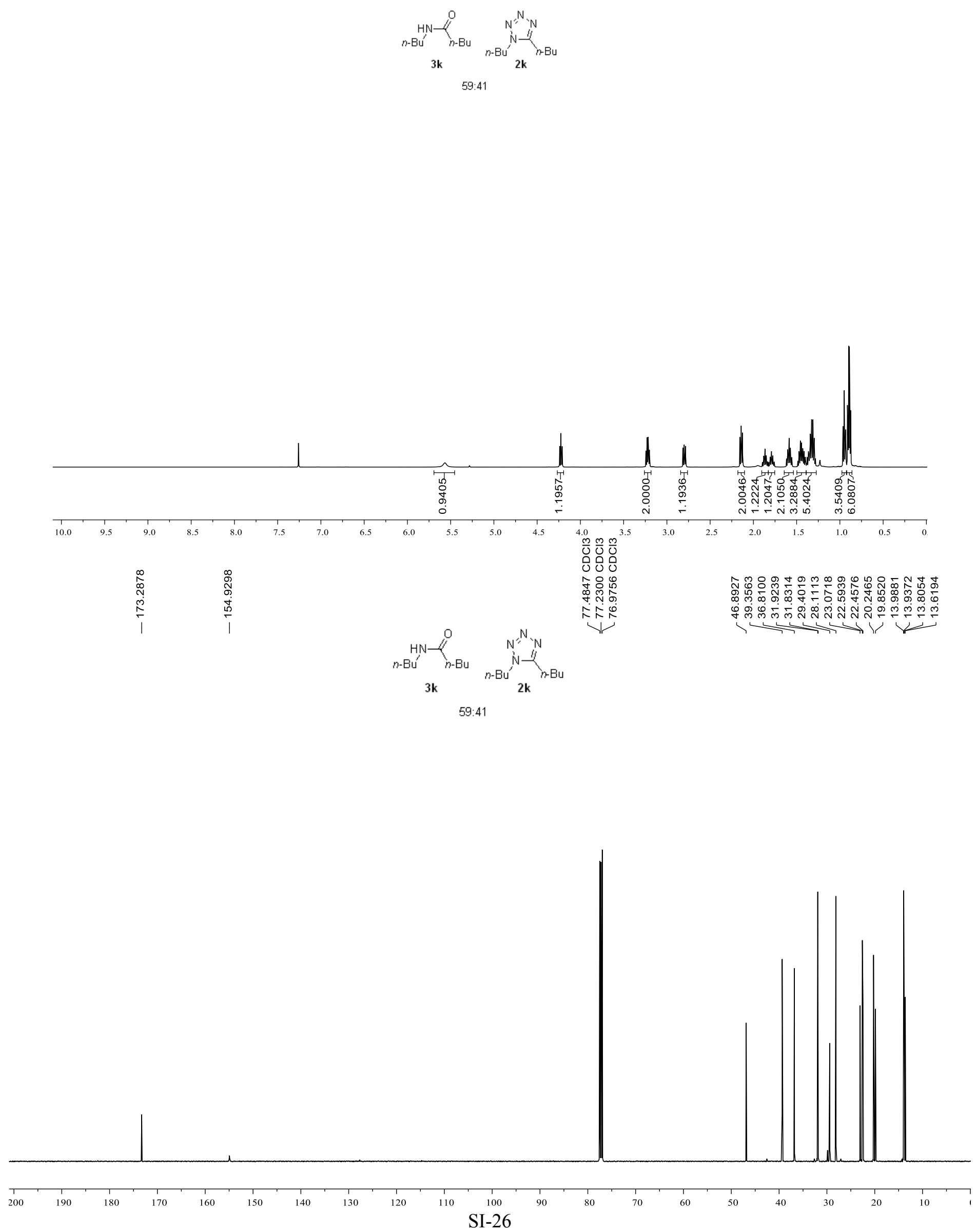

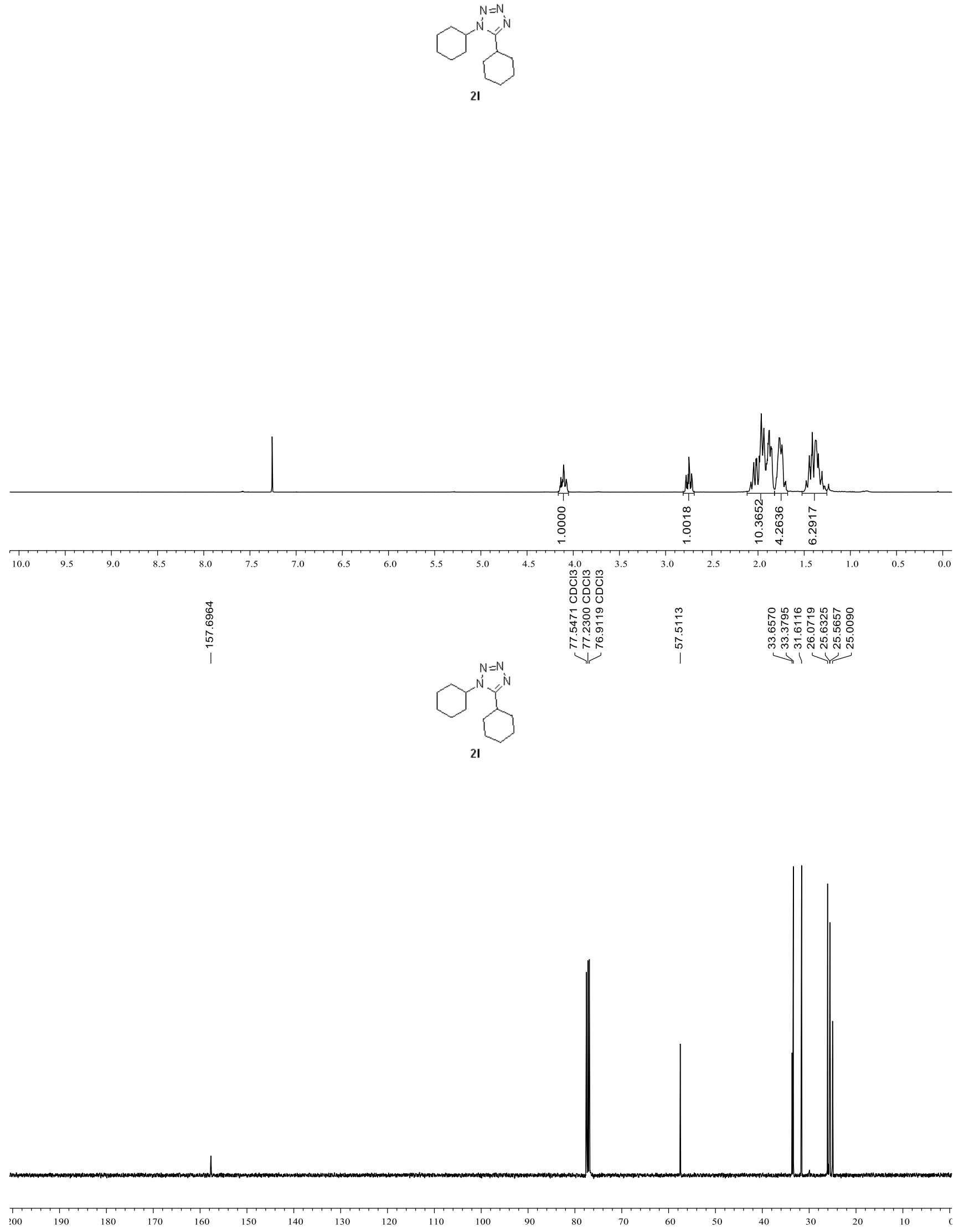


$$
\mathrm{Et}_{2 m}^{N / N}
$$
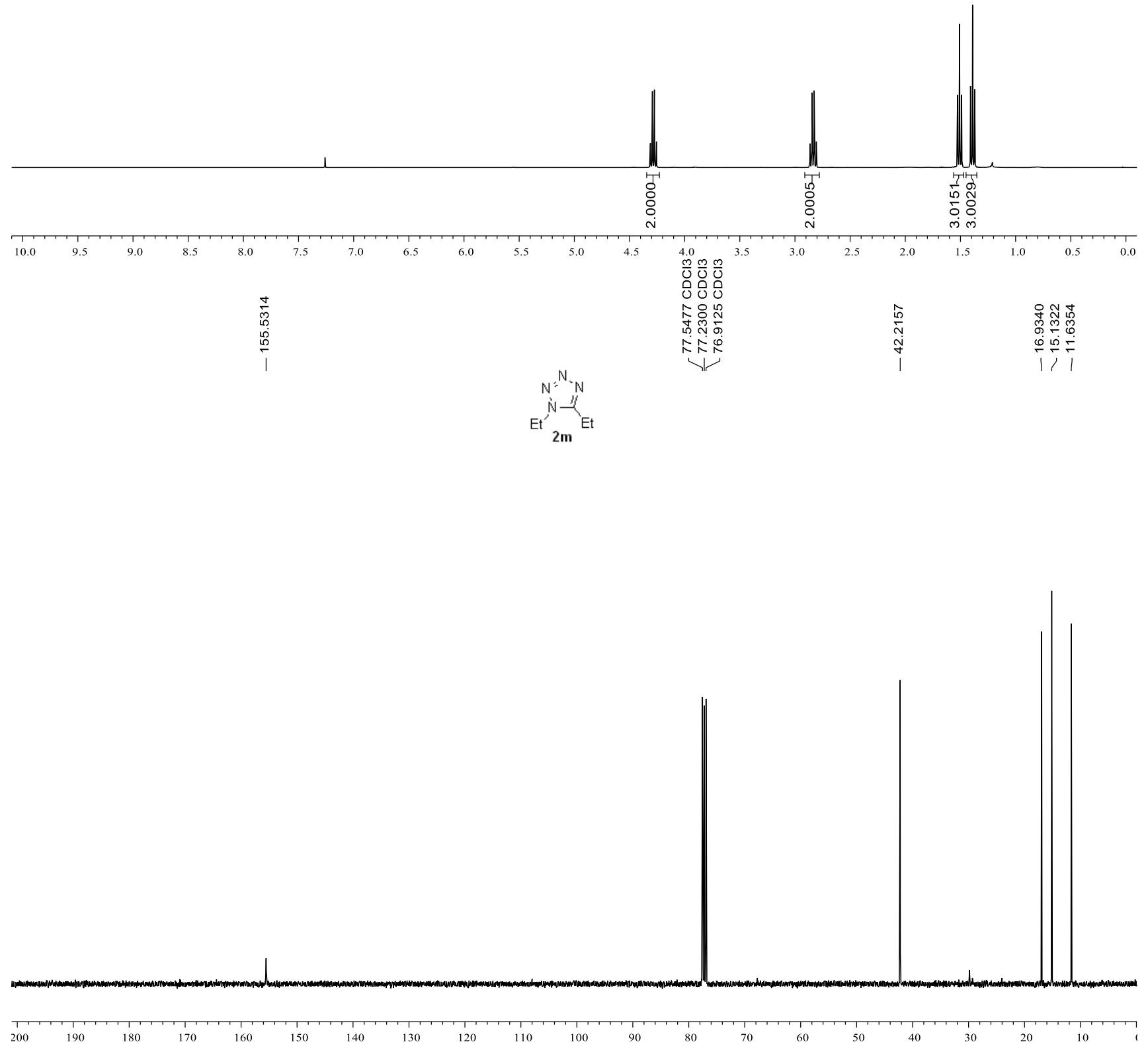

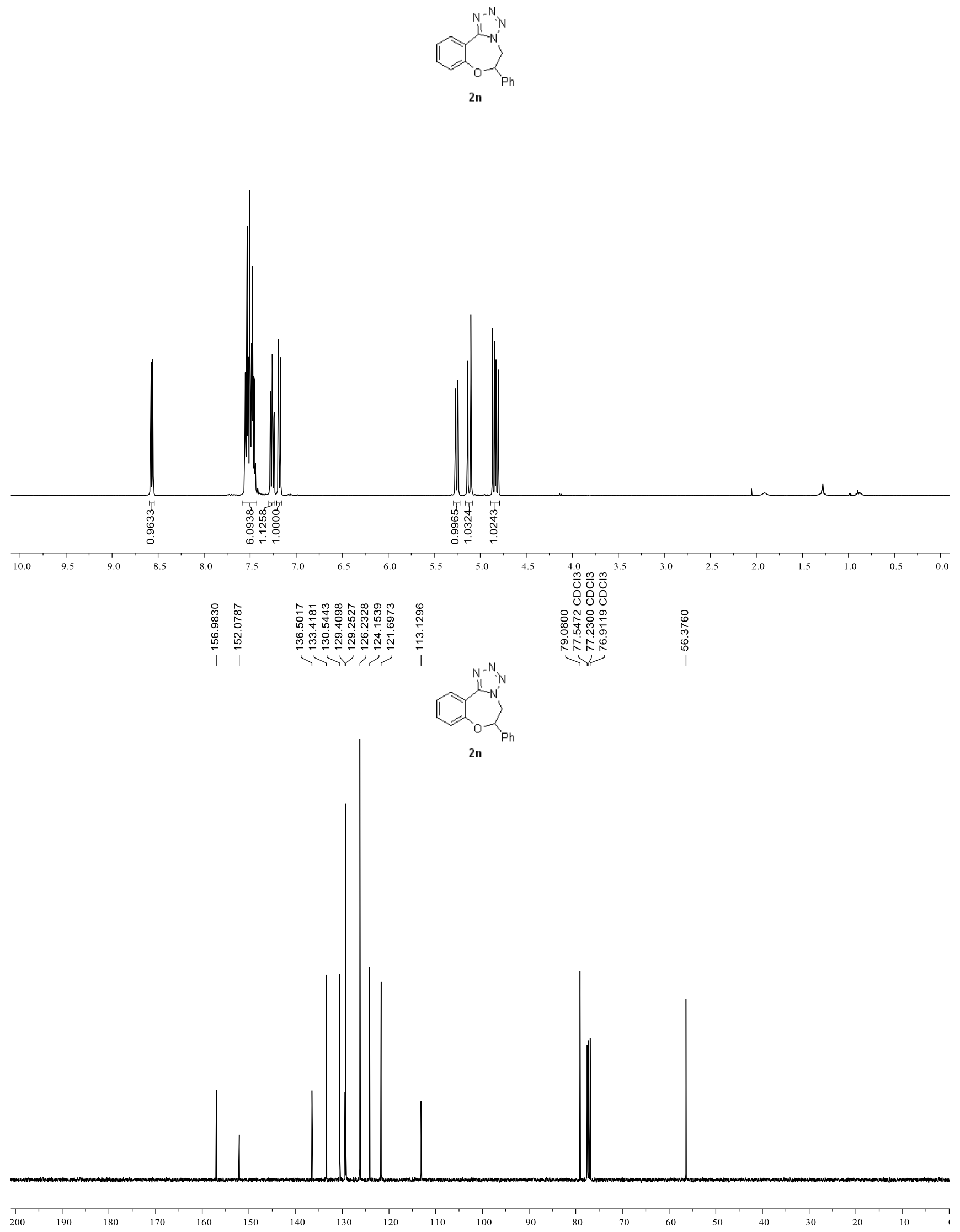


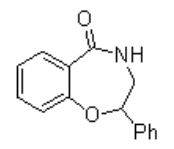

3na
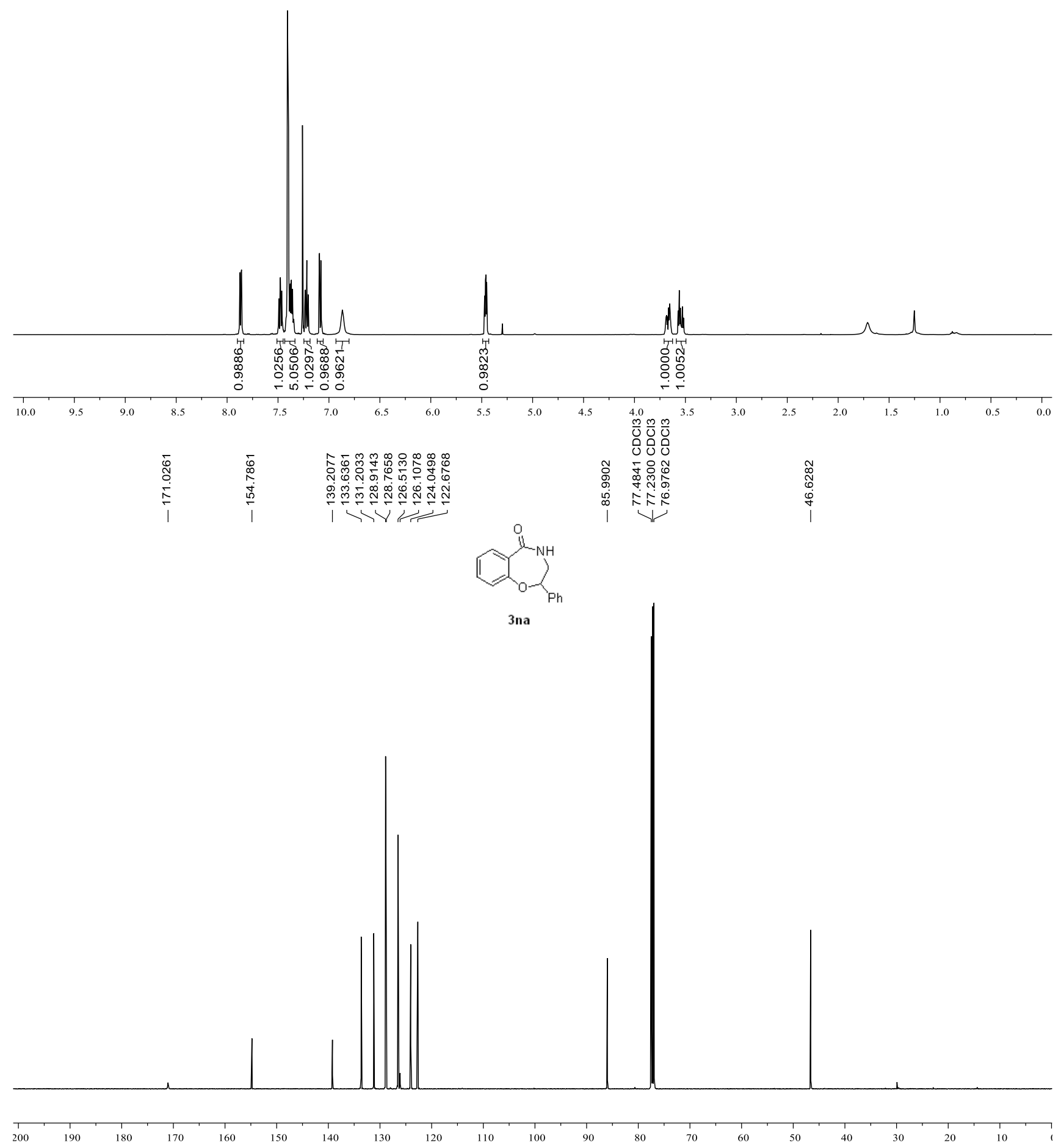

0
0
0
6
0
0
1 


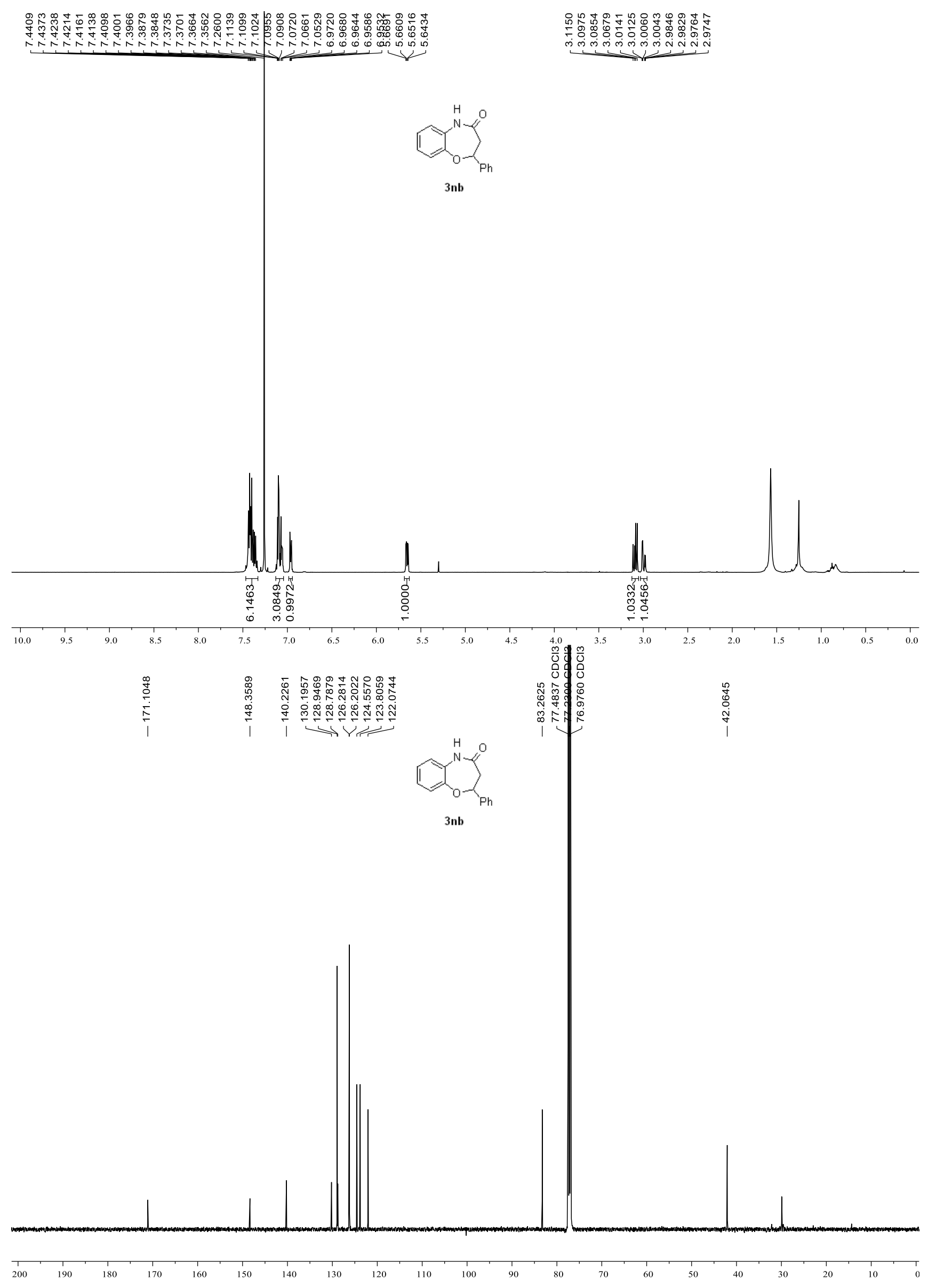

SI-31 

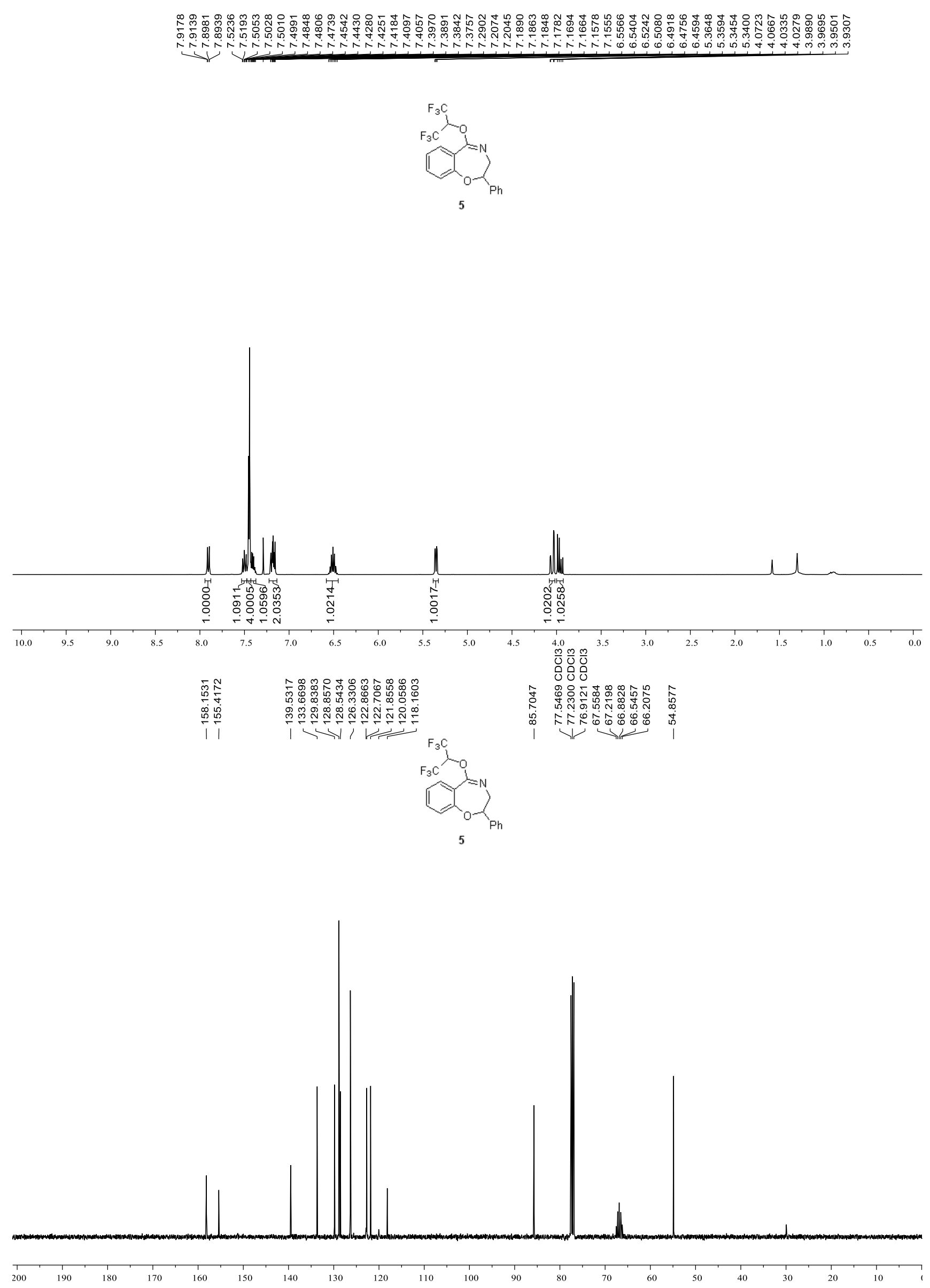

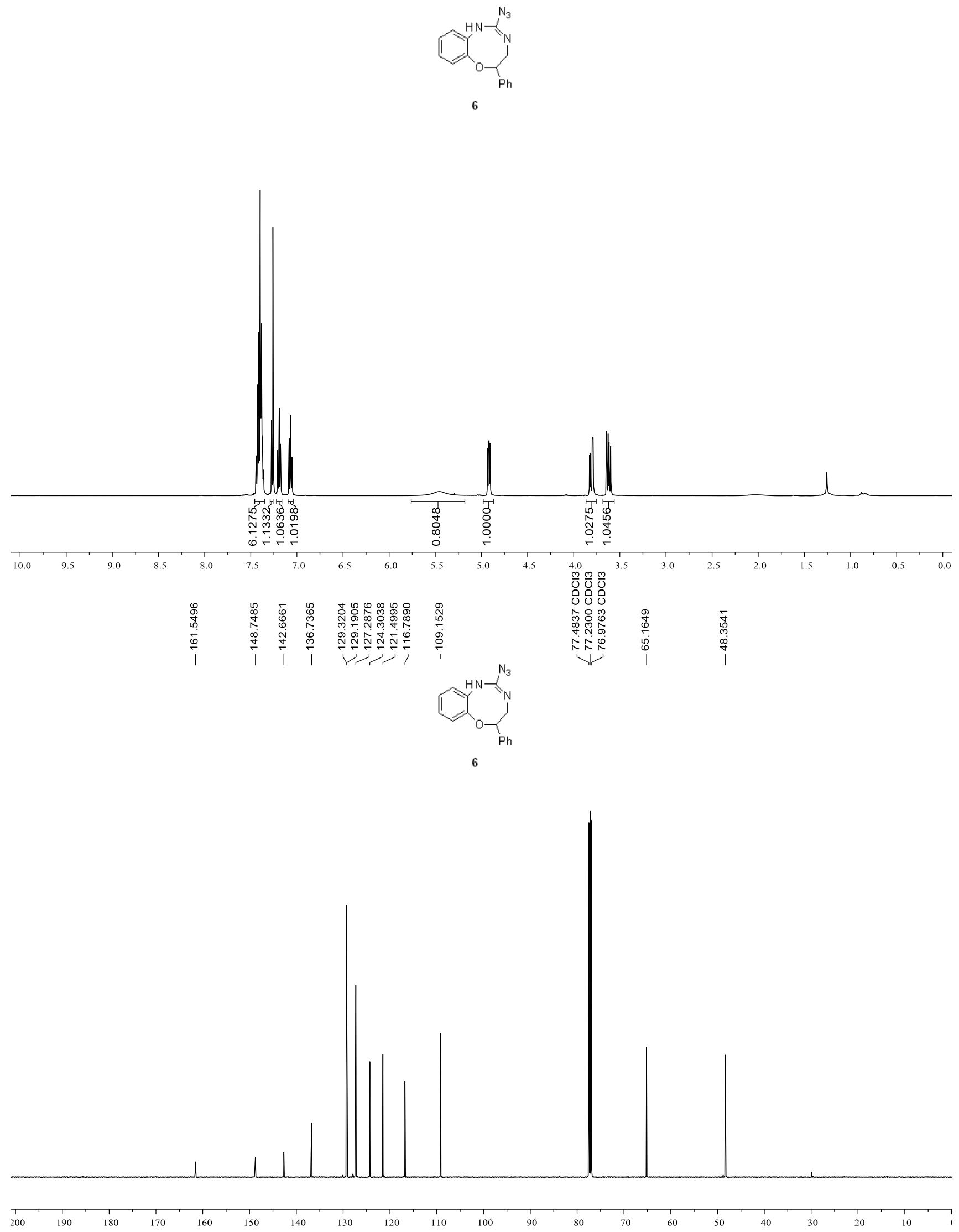


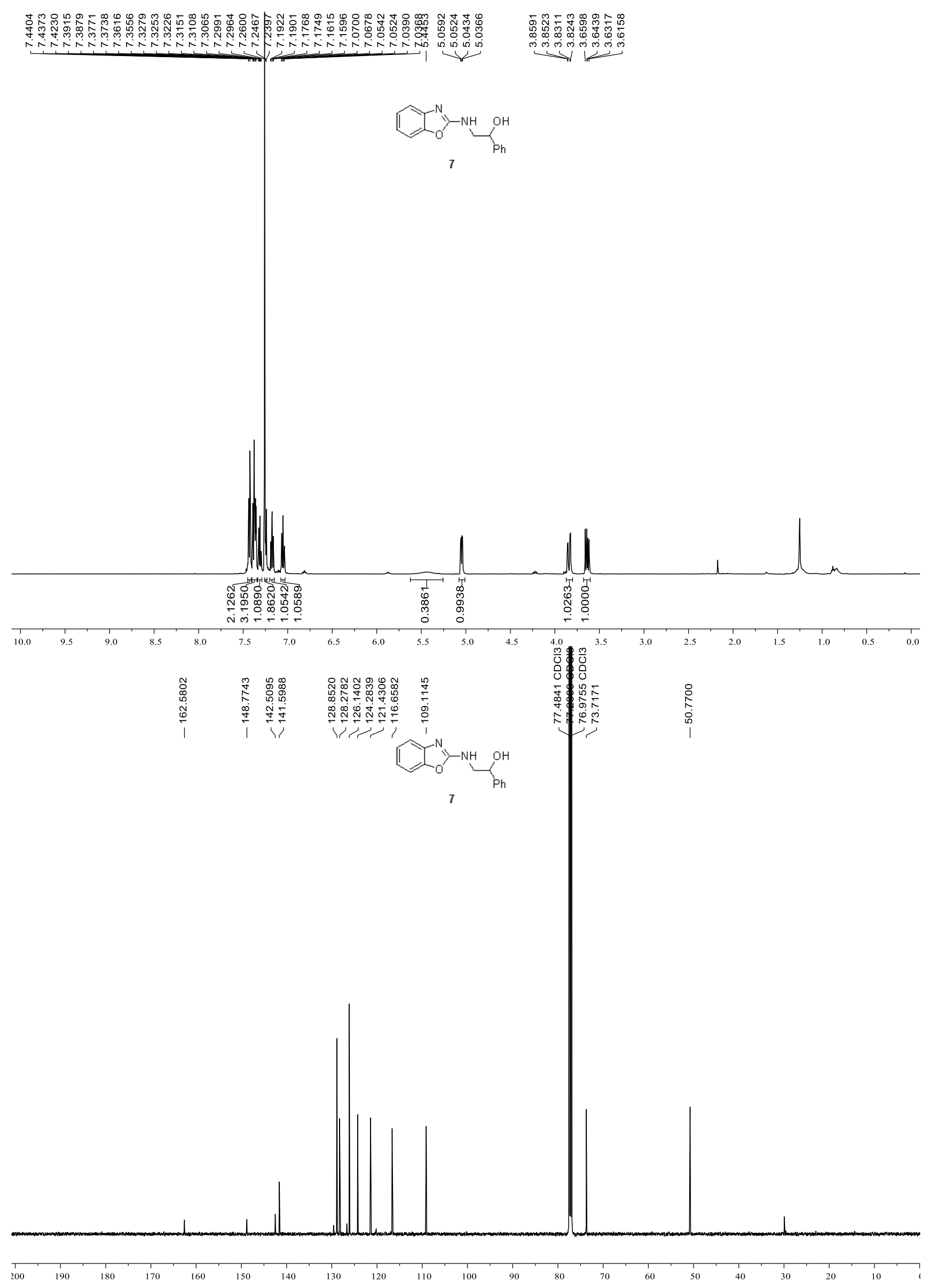



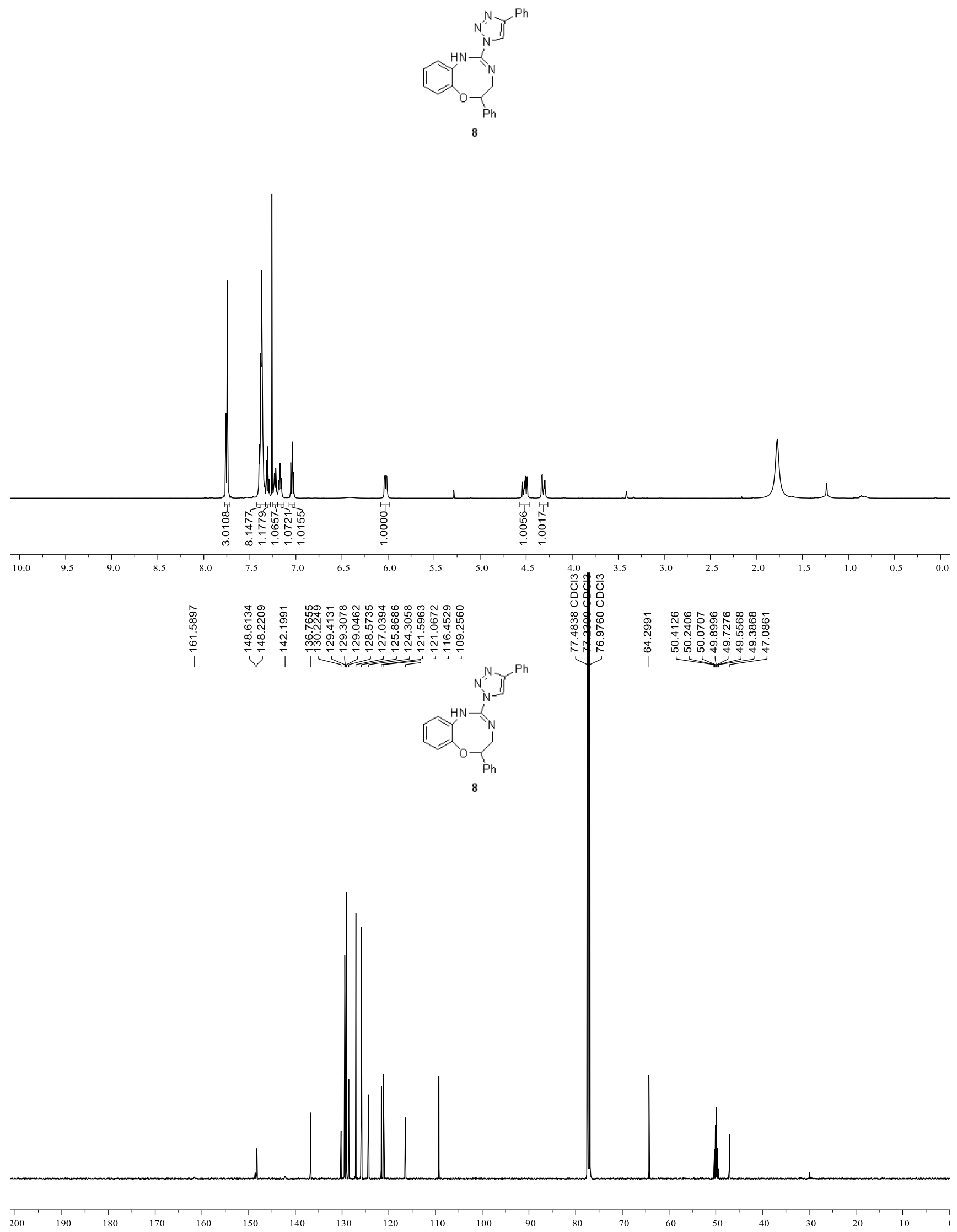

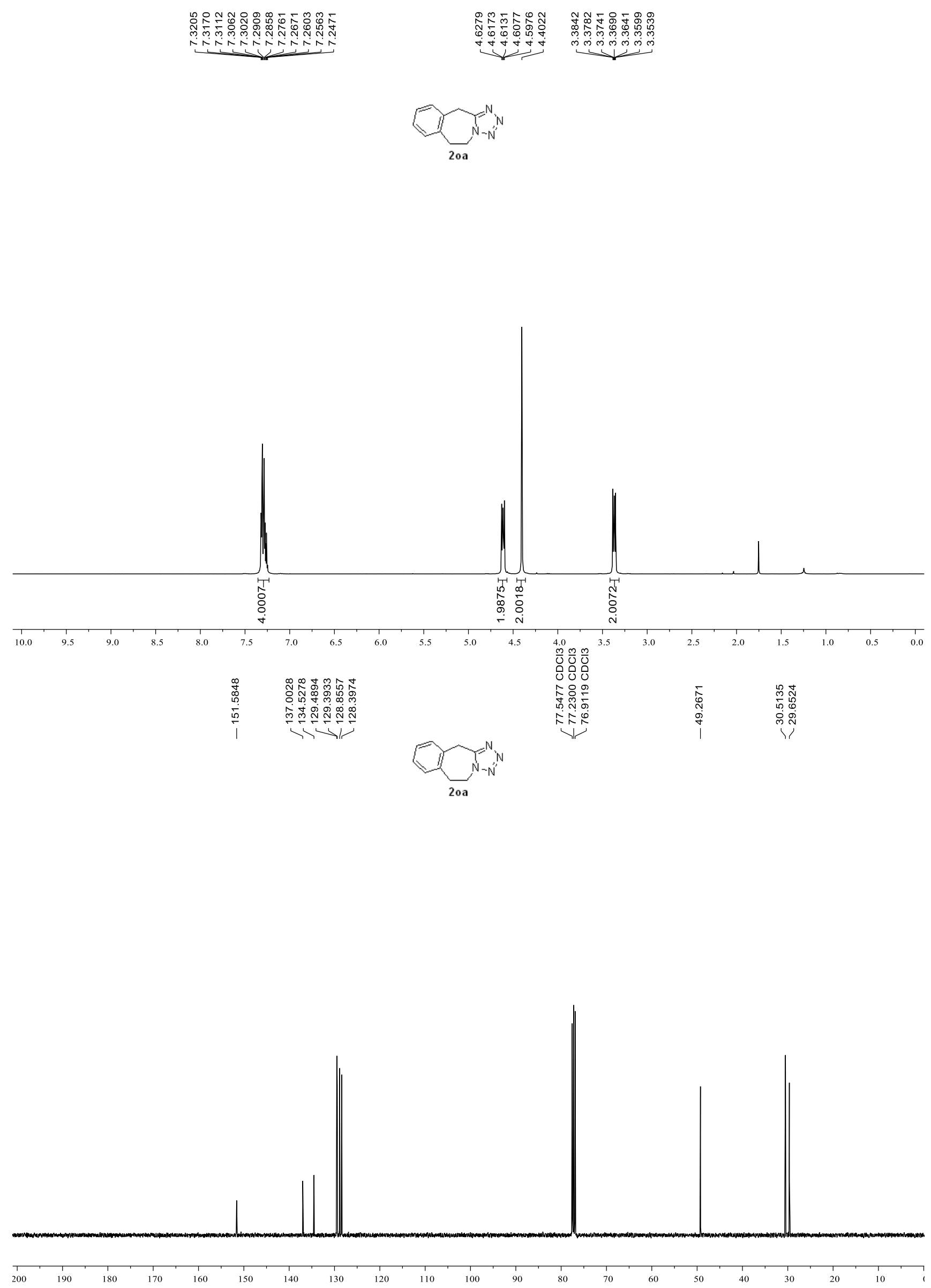

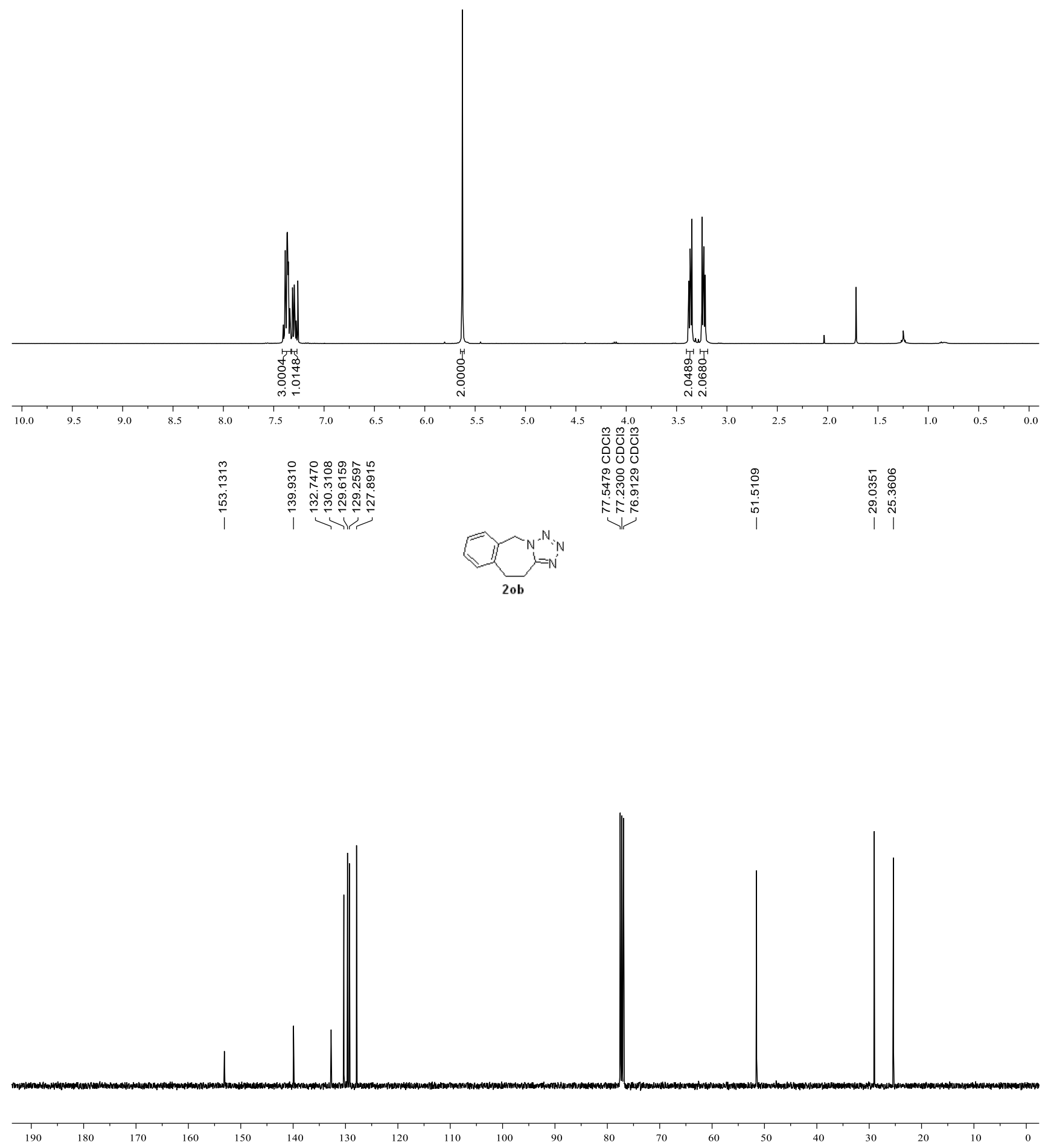


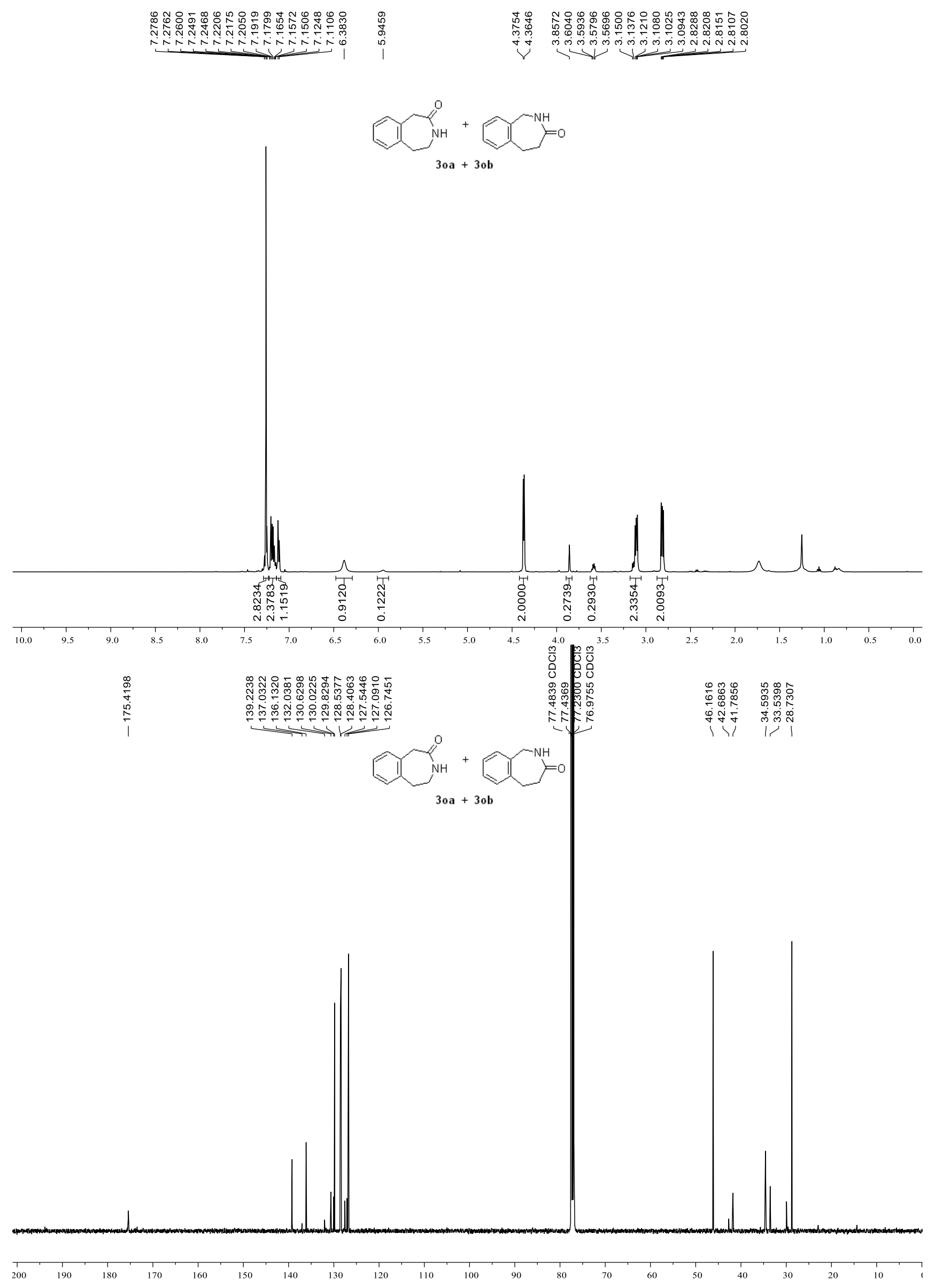




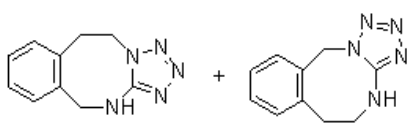

$40 a+4 o b$
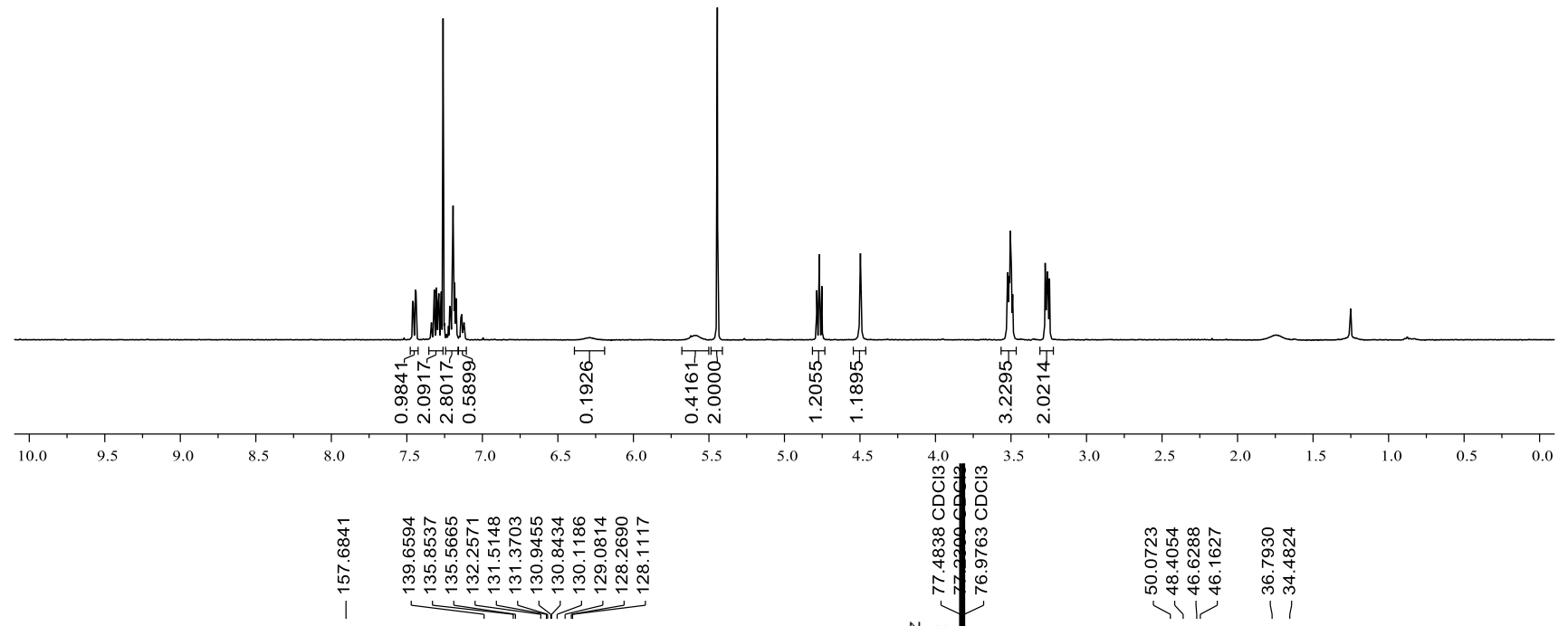

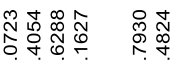

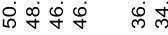

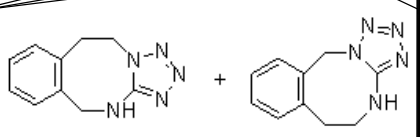

$40 a+40 b$

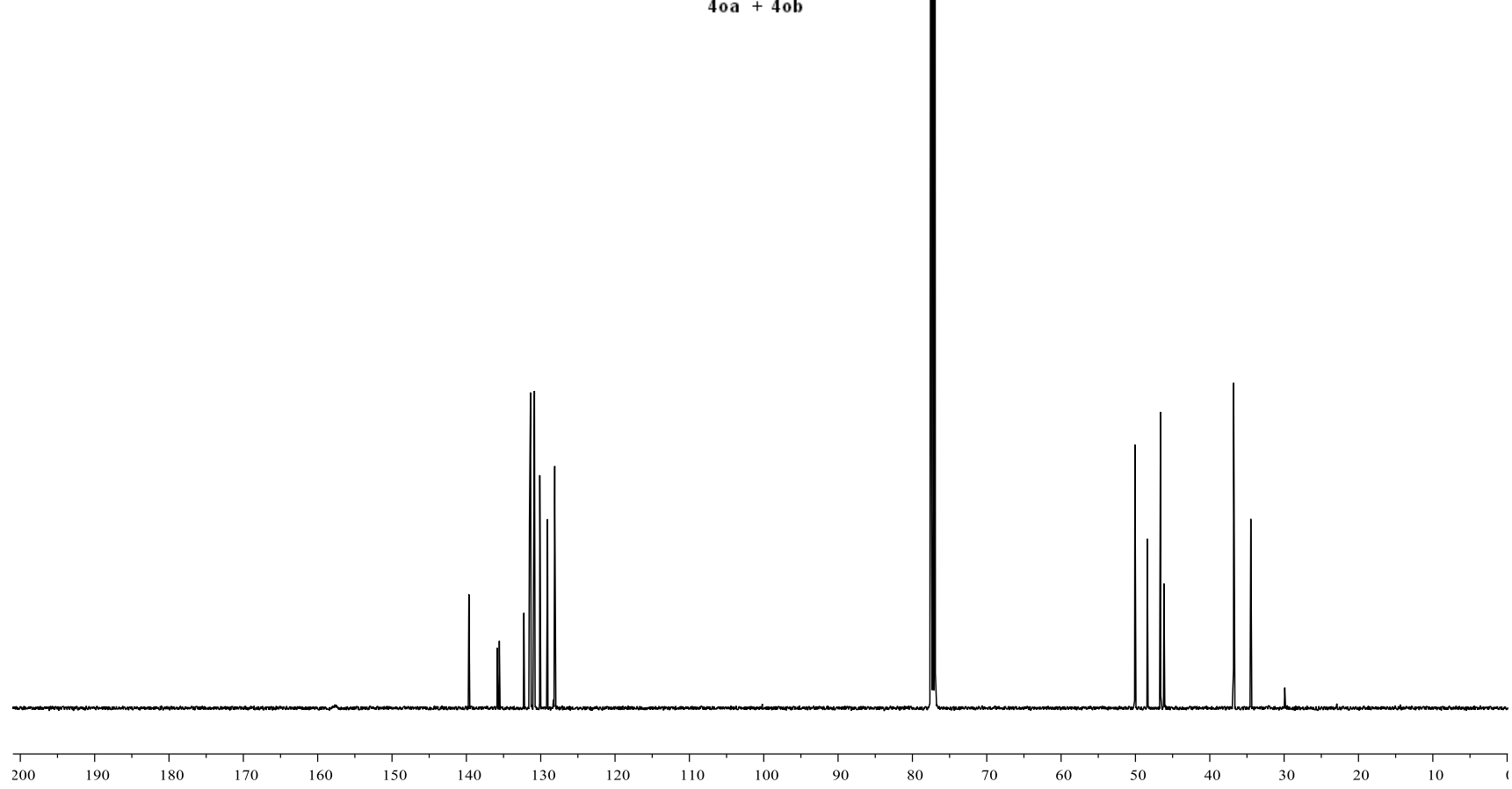



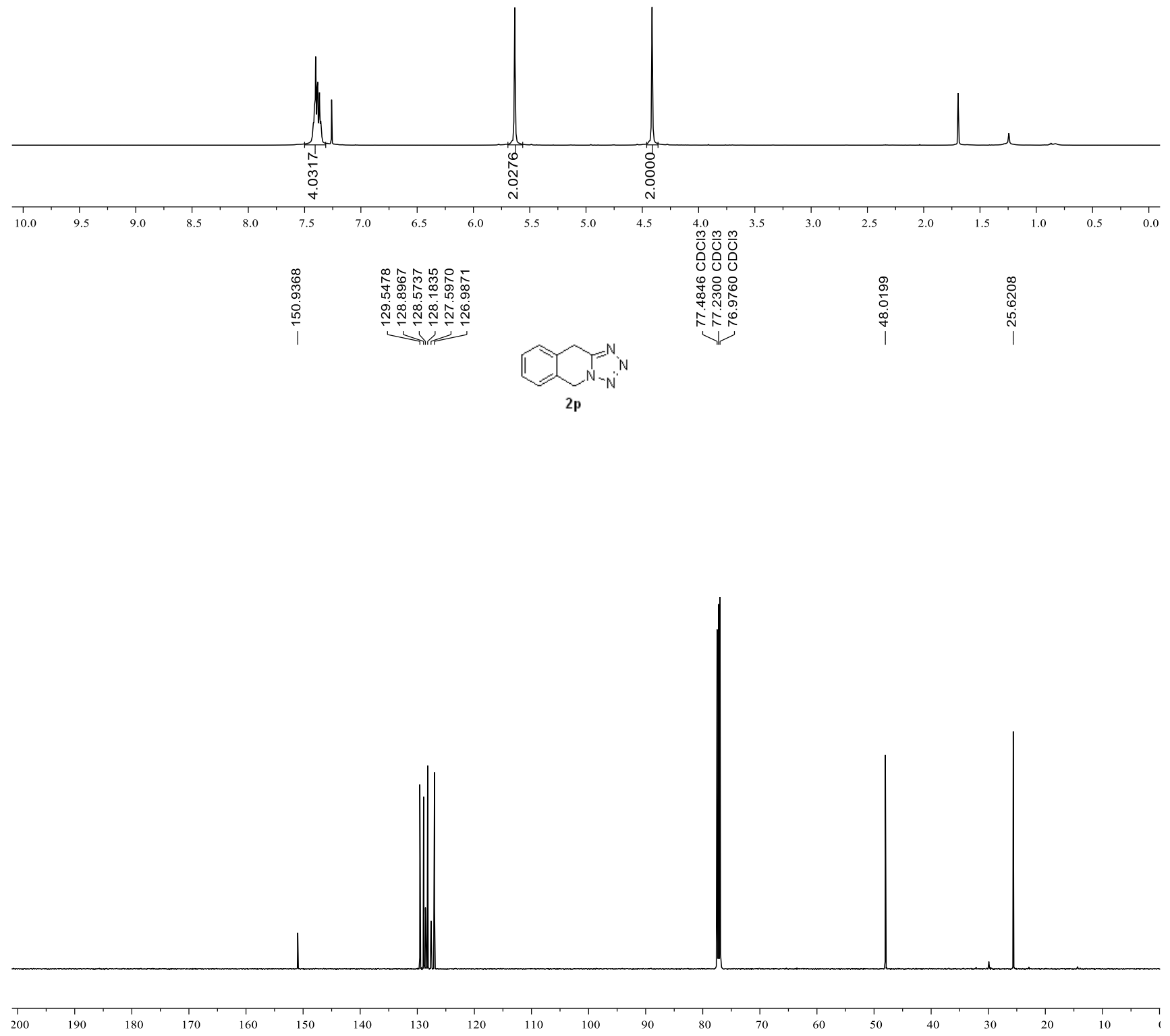

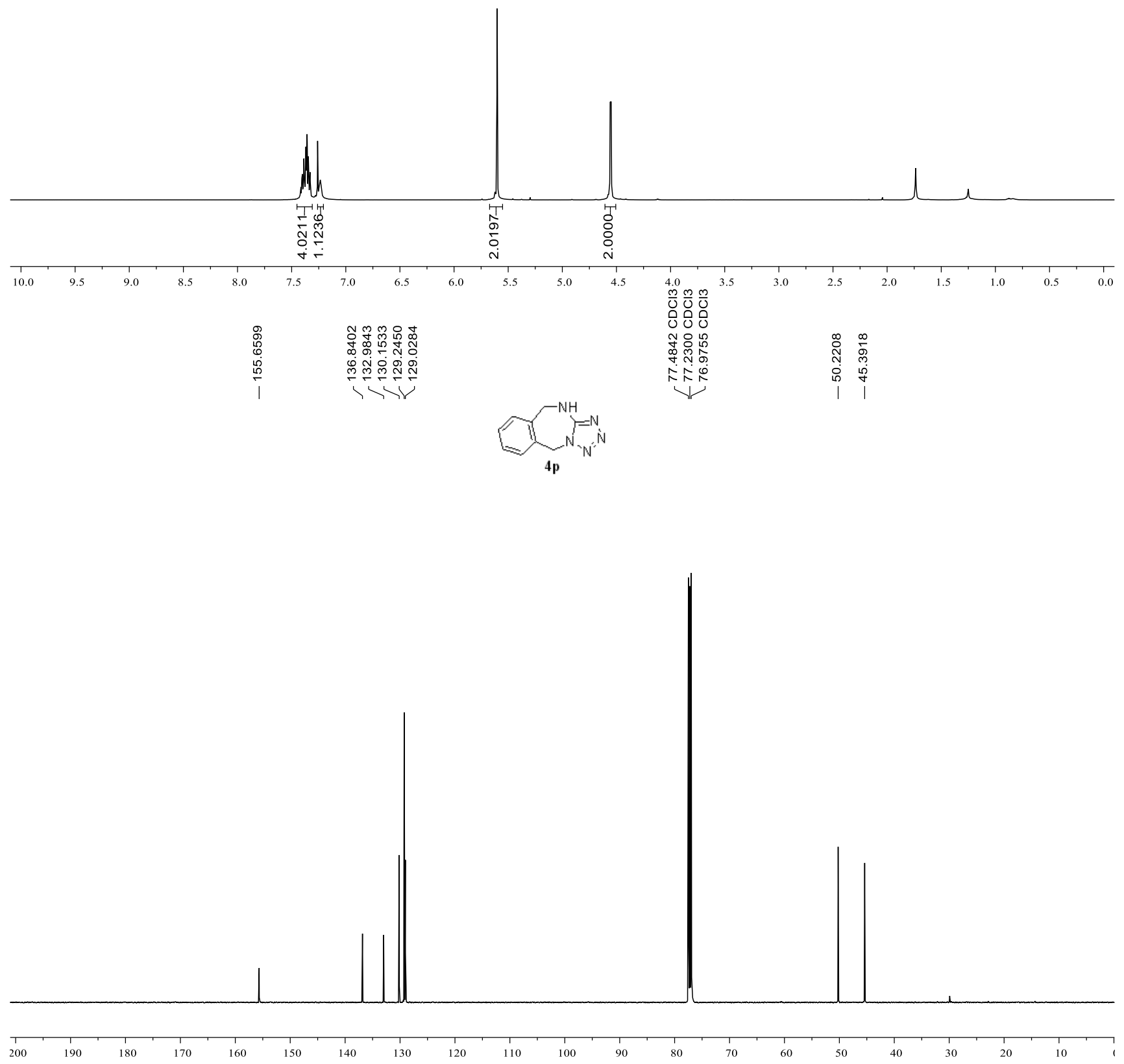

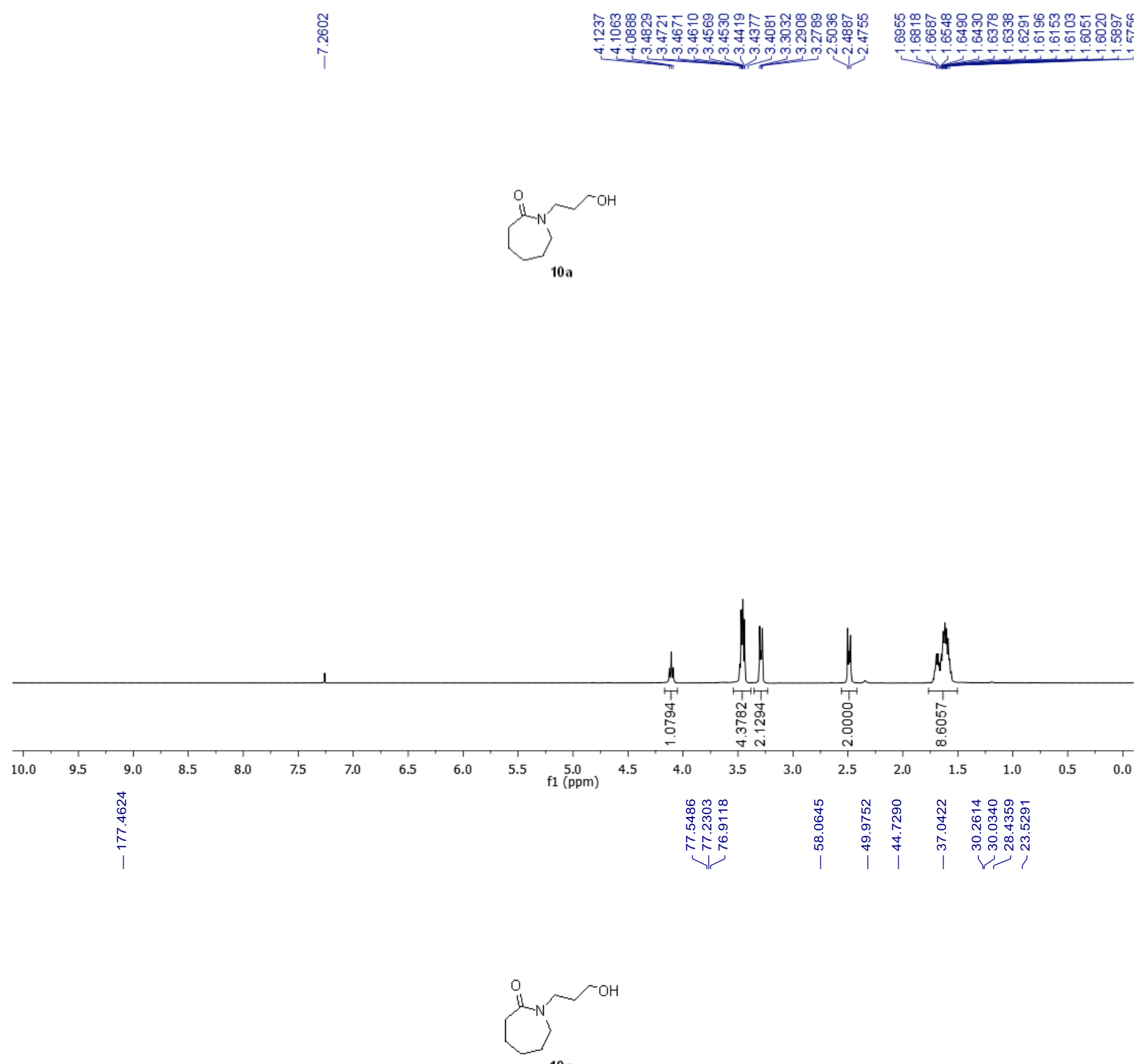

10 a

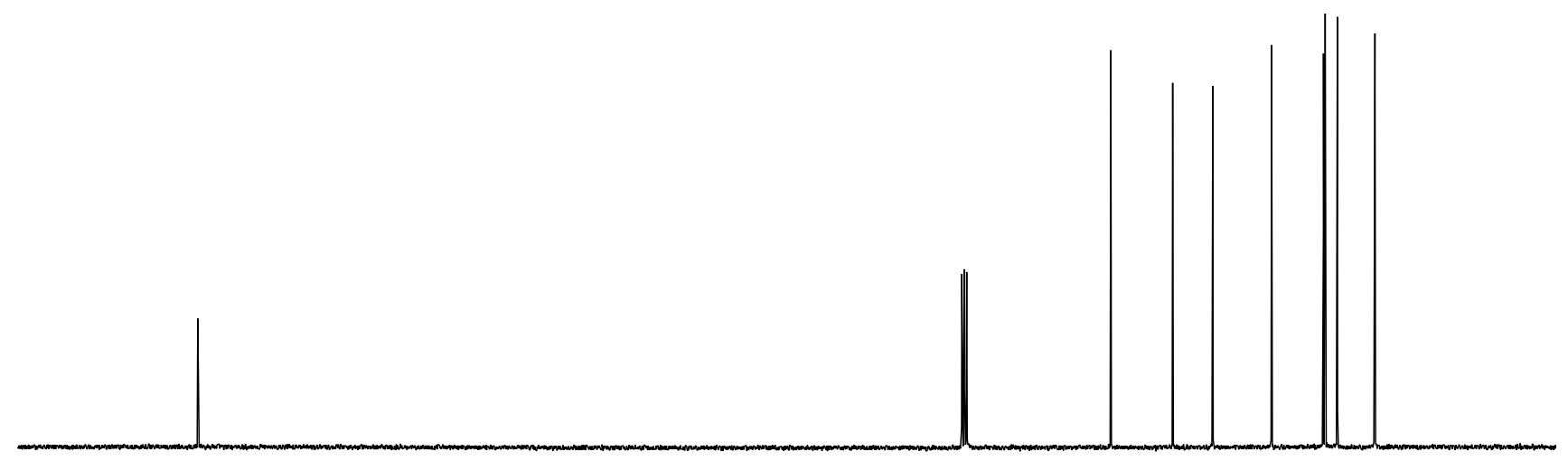

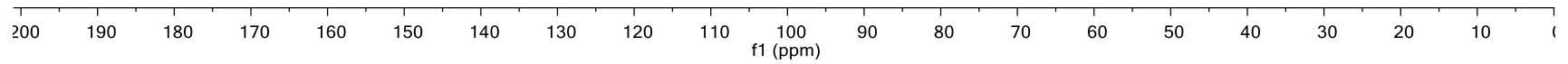



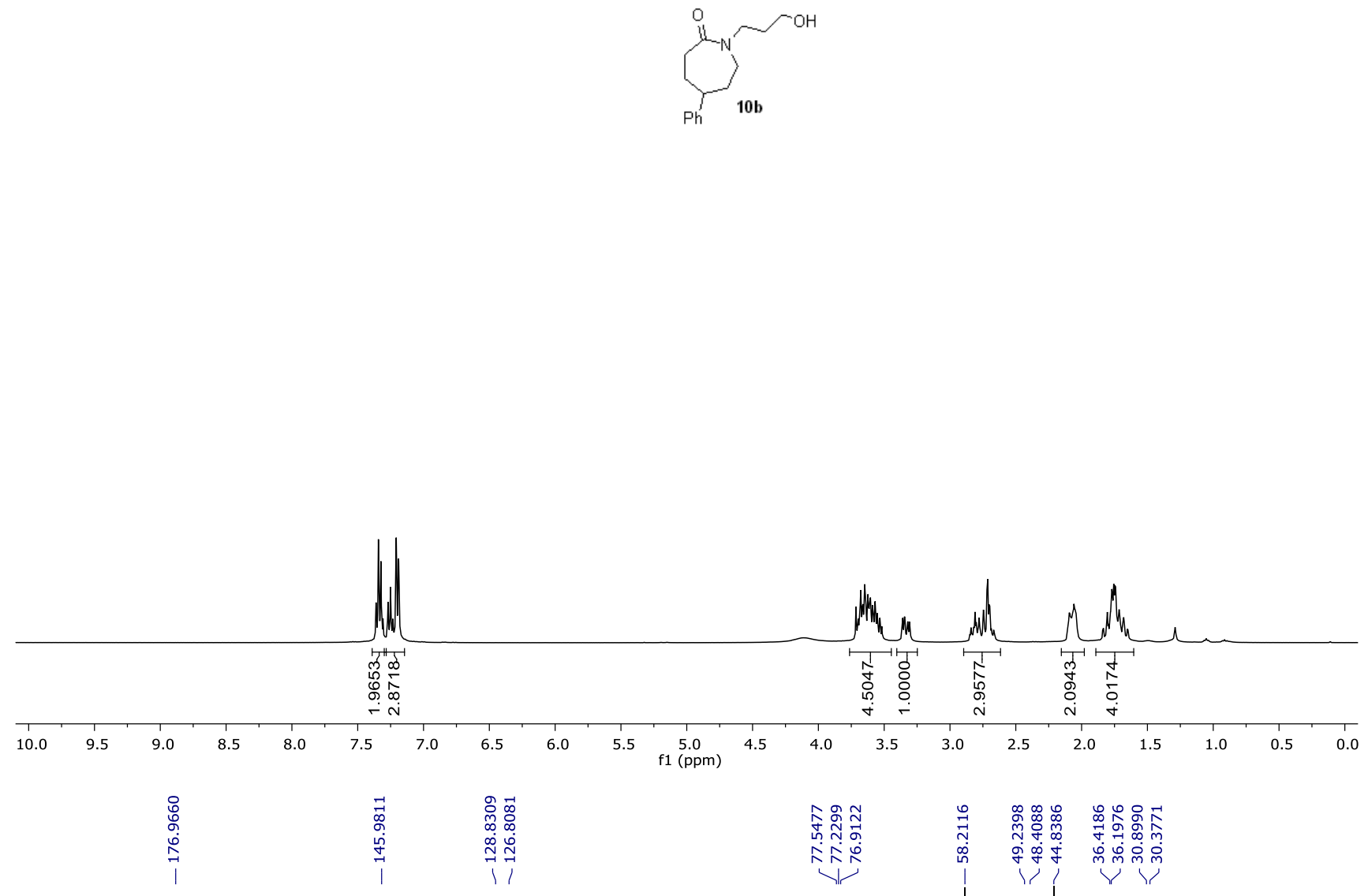

f
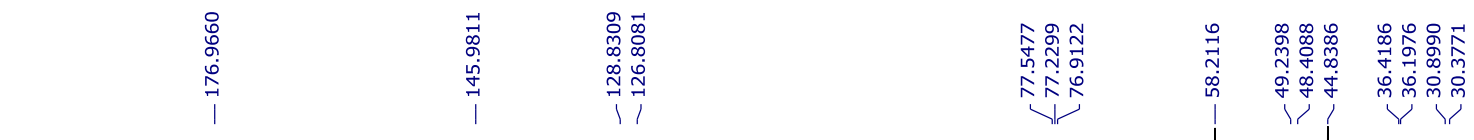

1
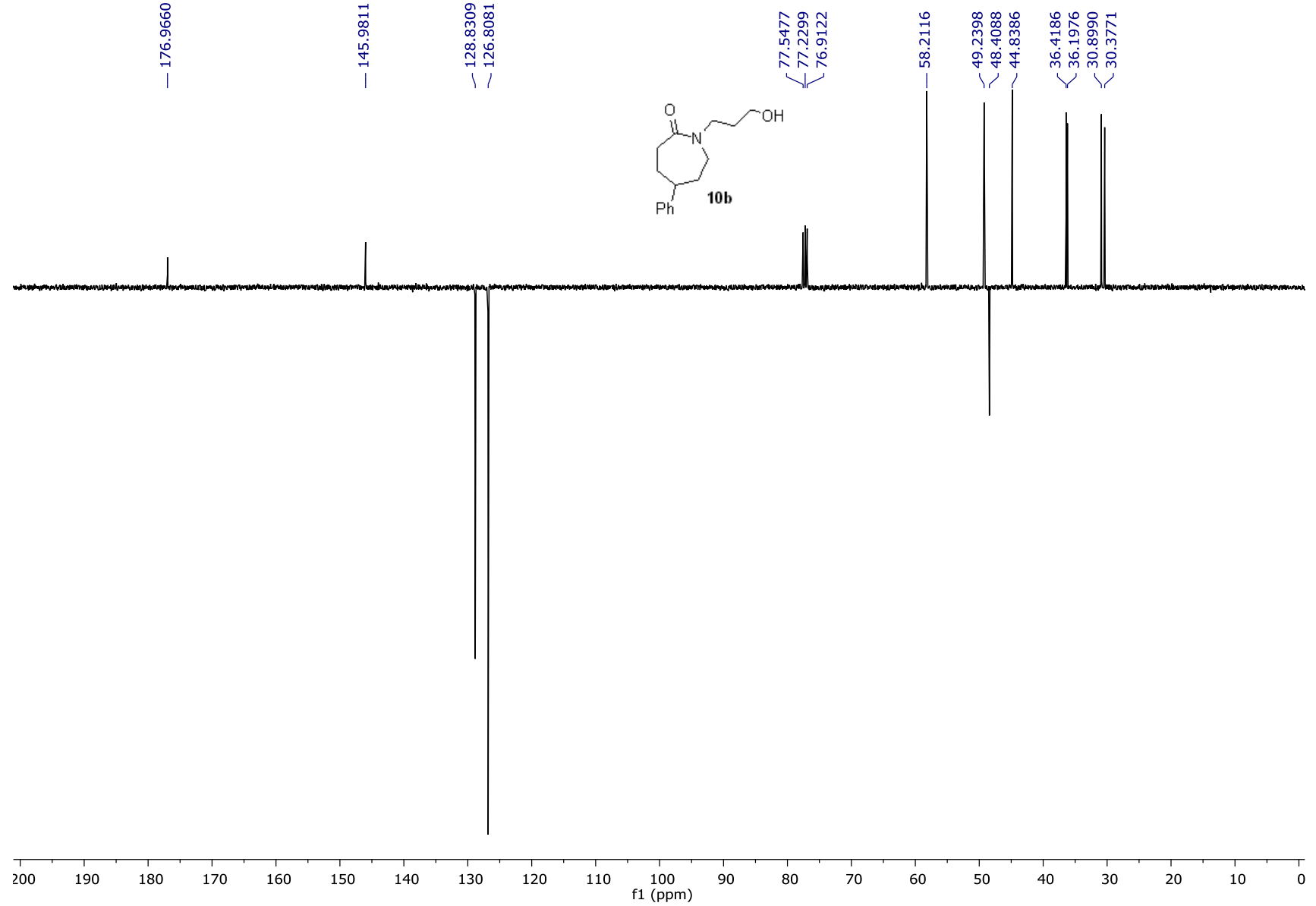


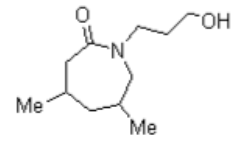

$10 \mathrm{~d}$

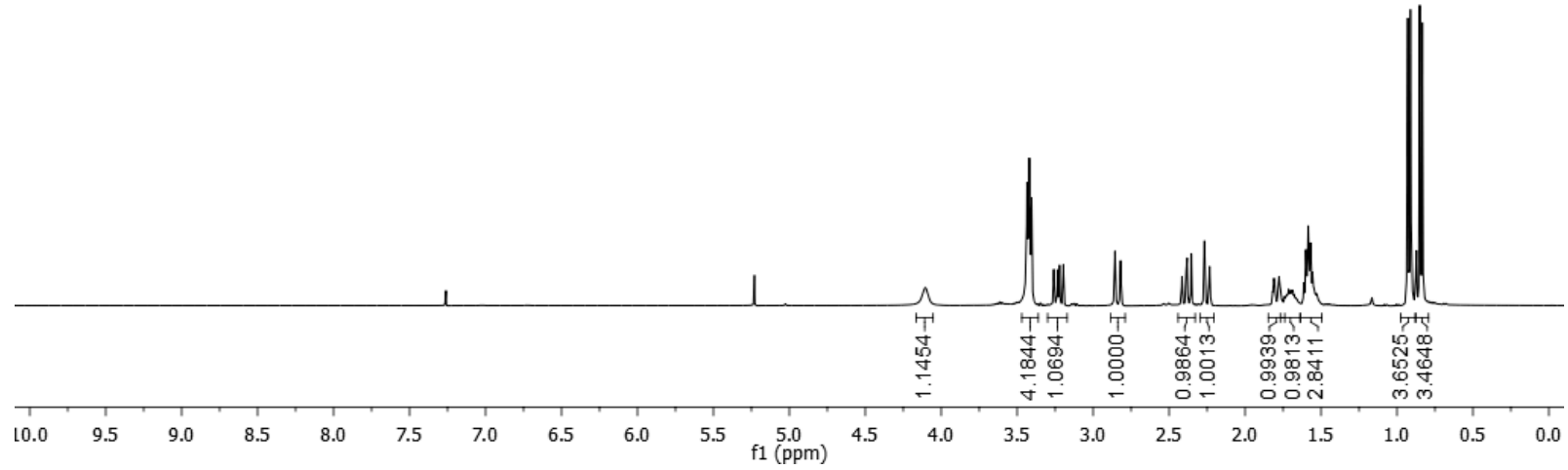

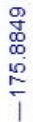

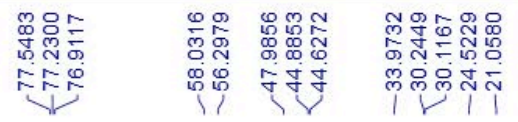

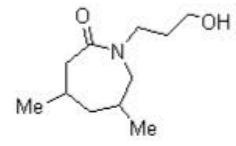

$10 \mathrm{~d}$

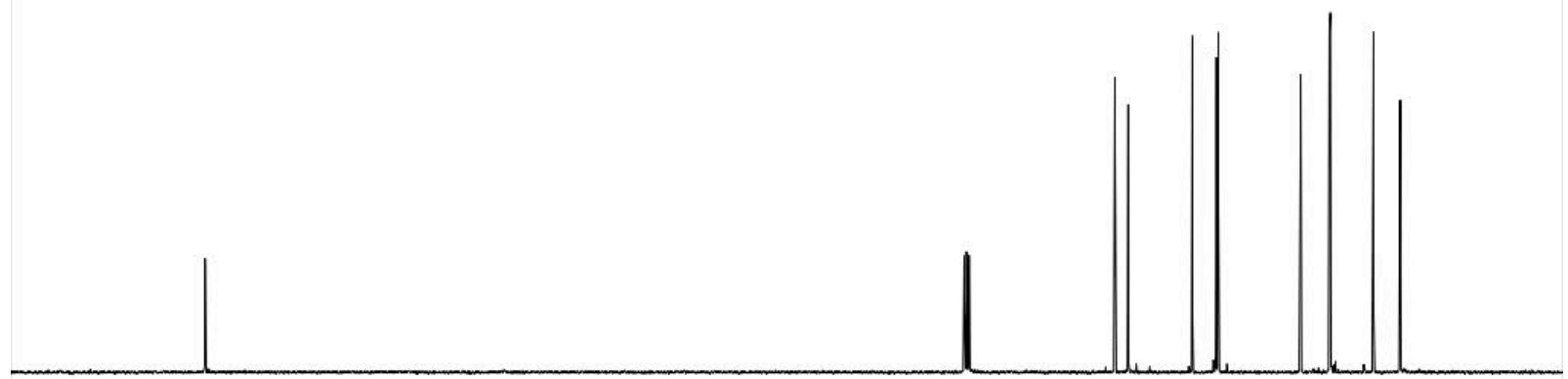

\begin{tabular}{|c|c|c|c|c|c|c|c|c|c|c|c|c|c|c|c|c|c|c|c|}
\hline 00 & 190 & 180 & 170 & 160 & 150 & 140 & 130 & 120 & 110 & $\begin{array}{r}100 \\
\mathrm{f} 1 \\
(\mathrm{ppm})\end{array}$ & 90 & 80 & 70 & 60 & 50 & 40 & 30 & 20 & 10 \\
\hline
\end{tabular}




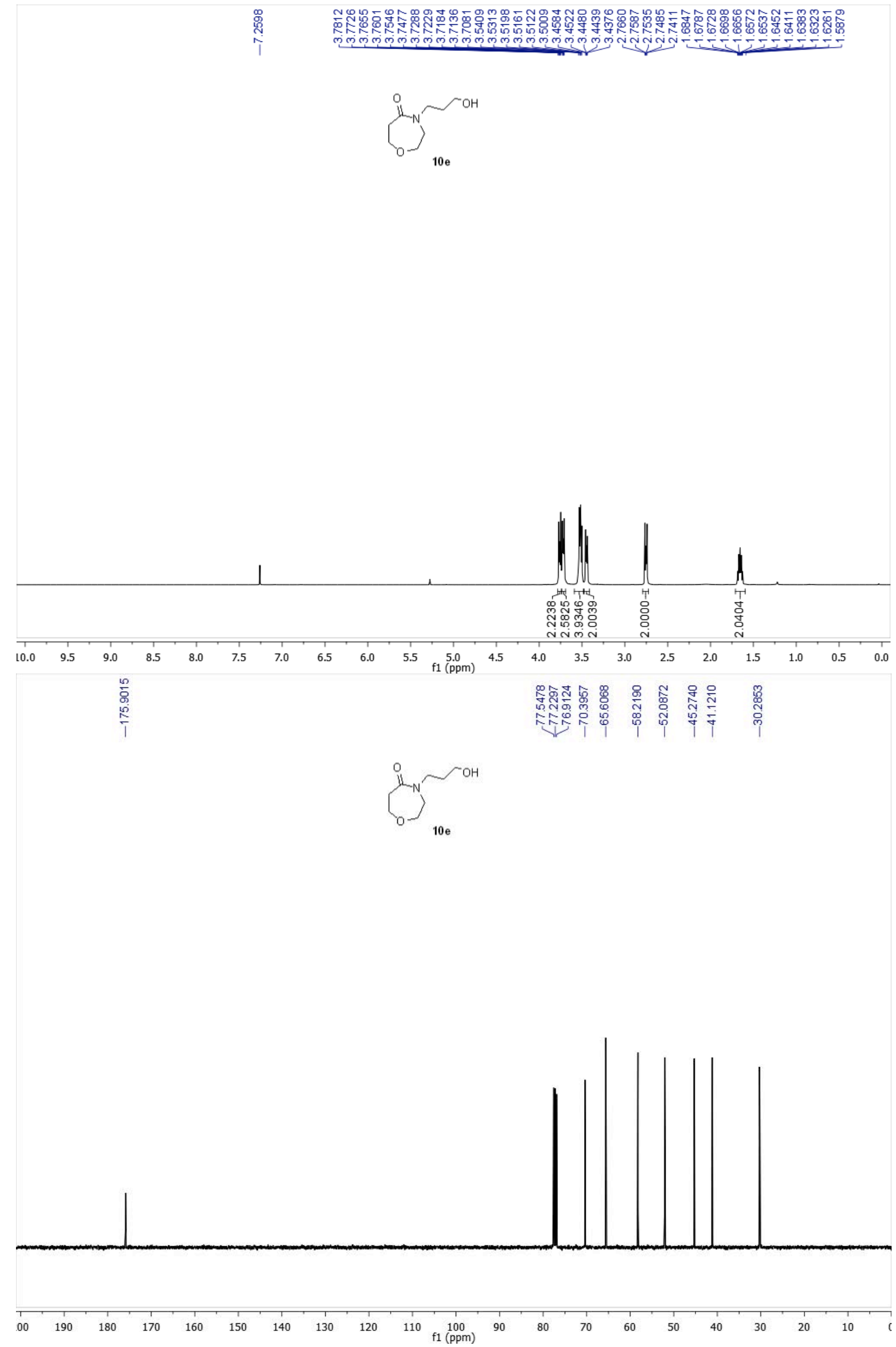




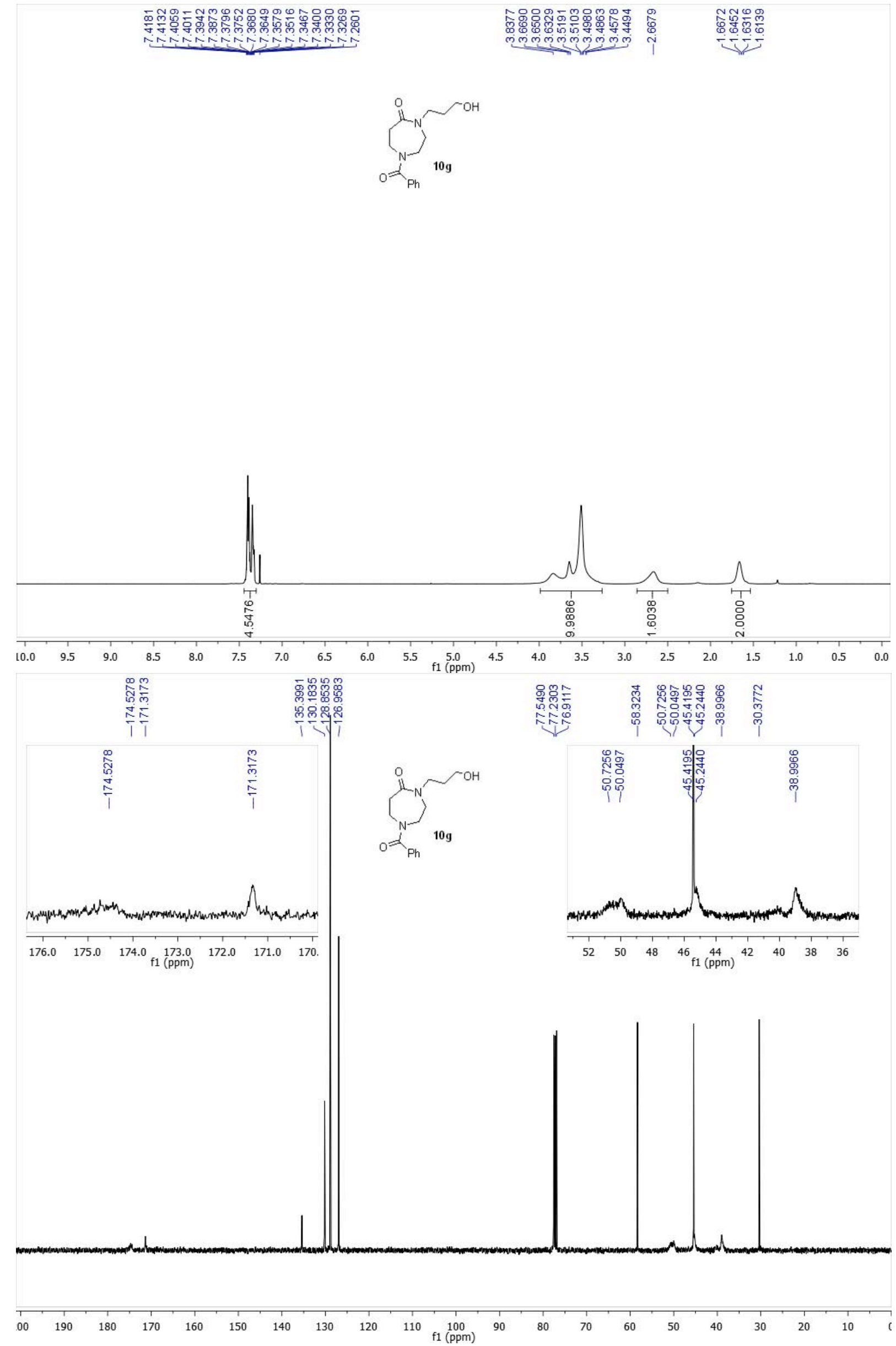




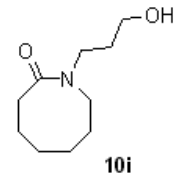

$10 \mathrm{i}$
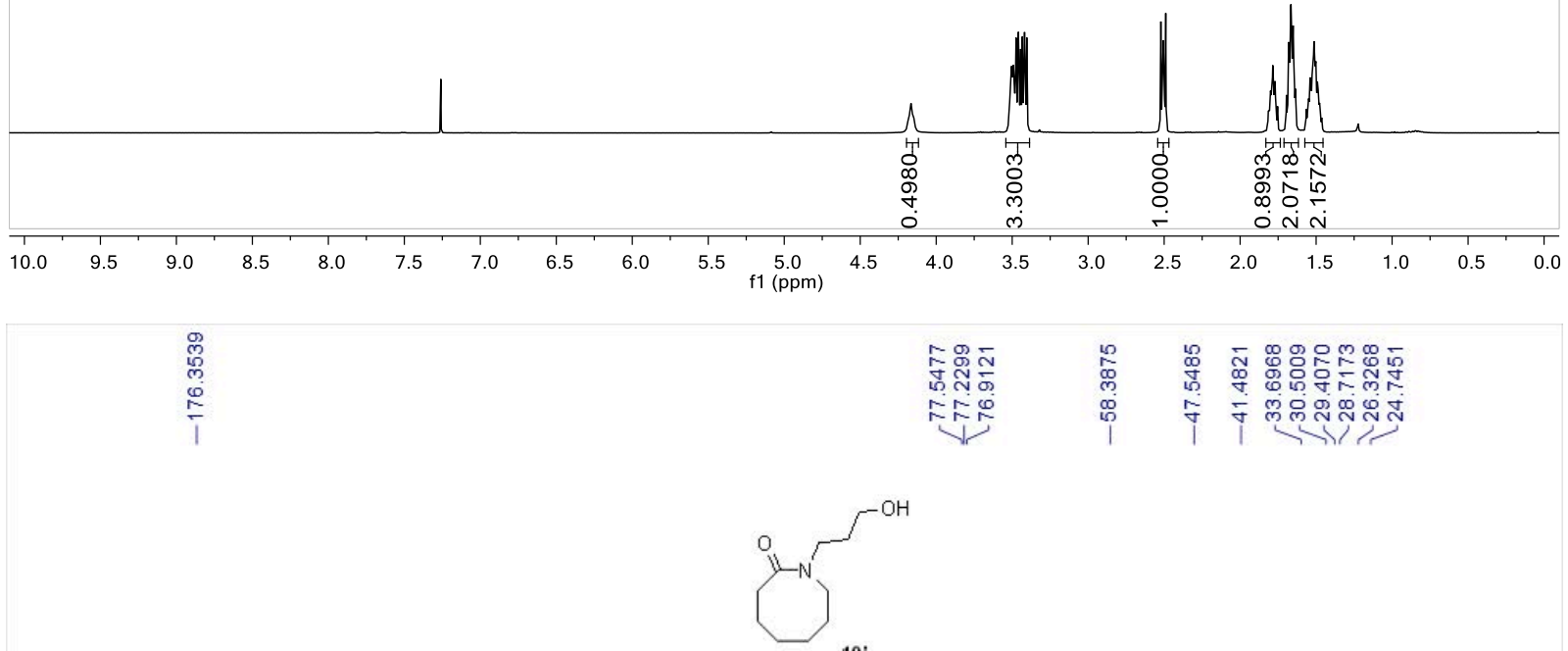

$10 \mathrm{i}$
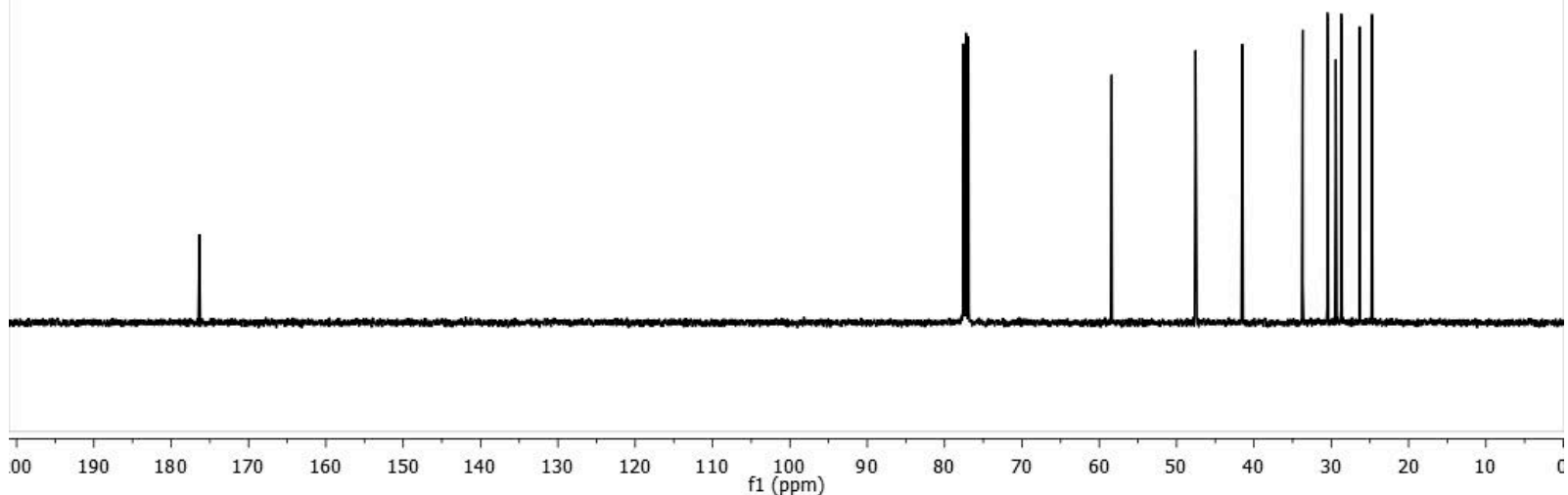


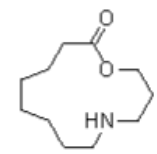

11j
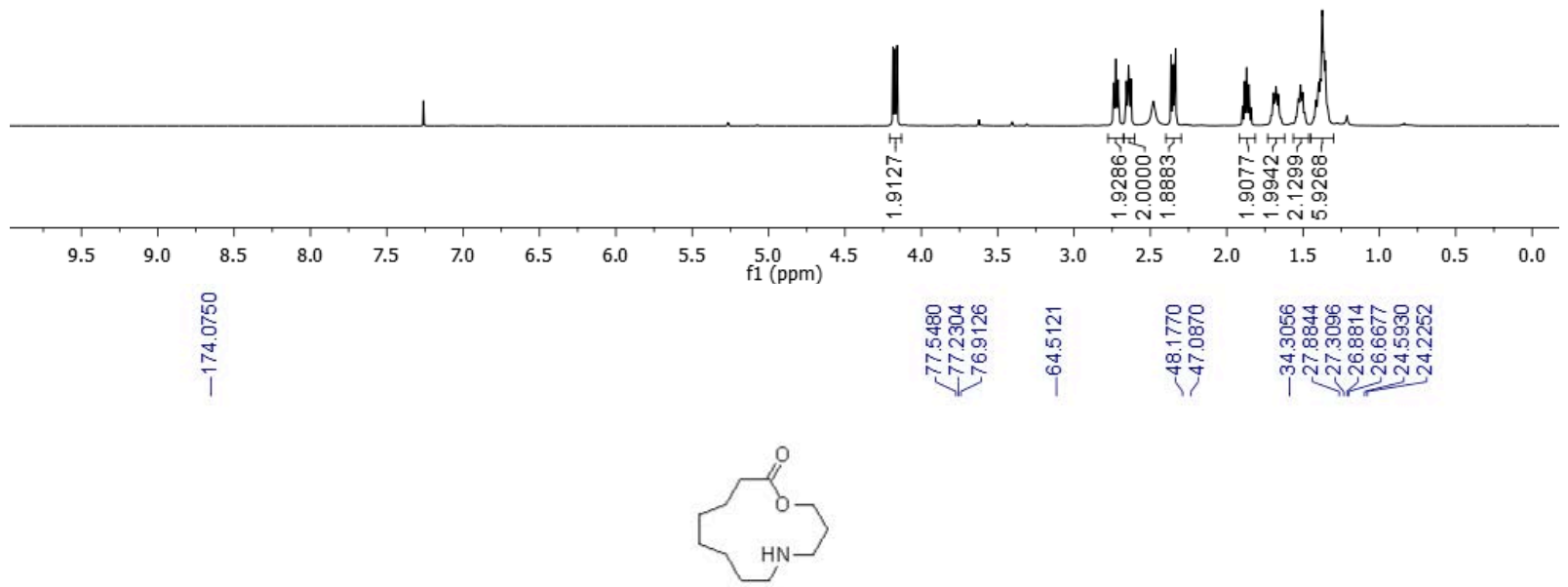

11j
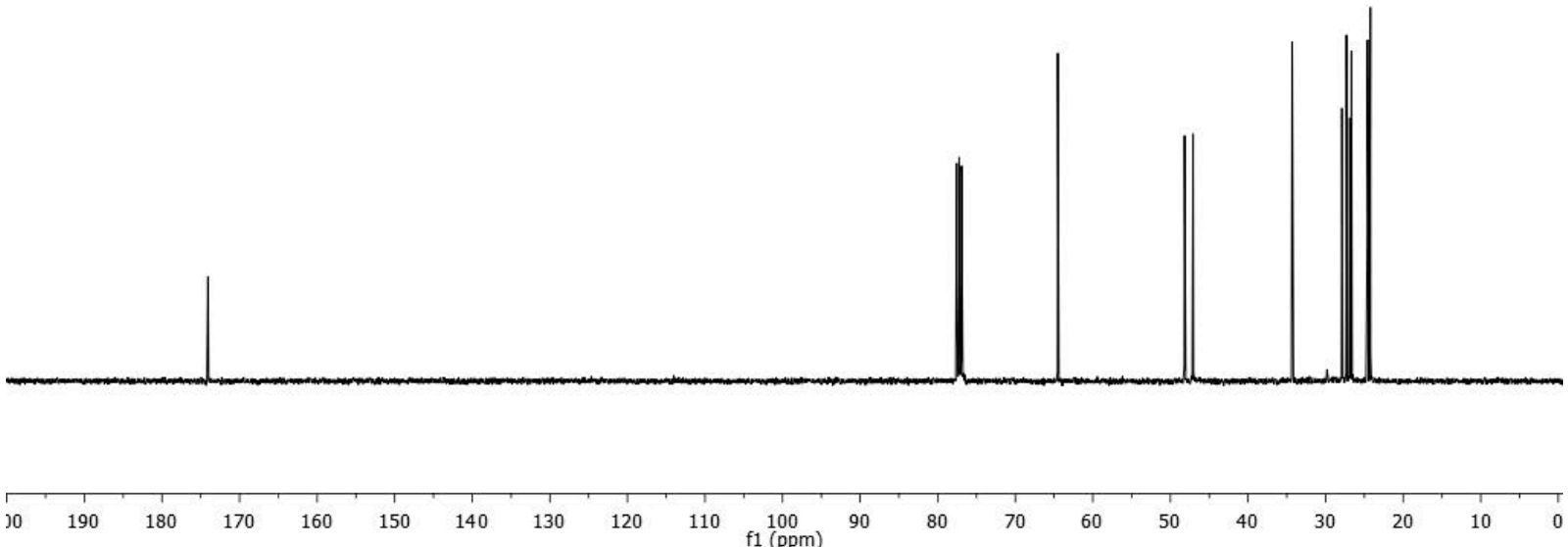

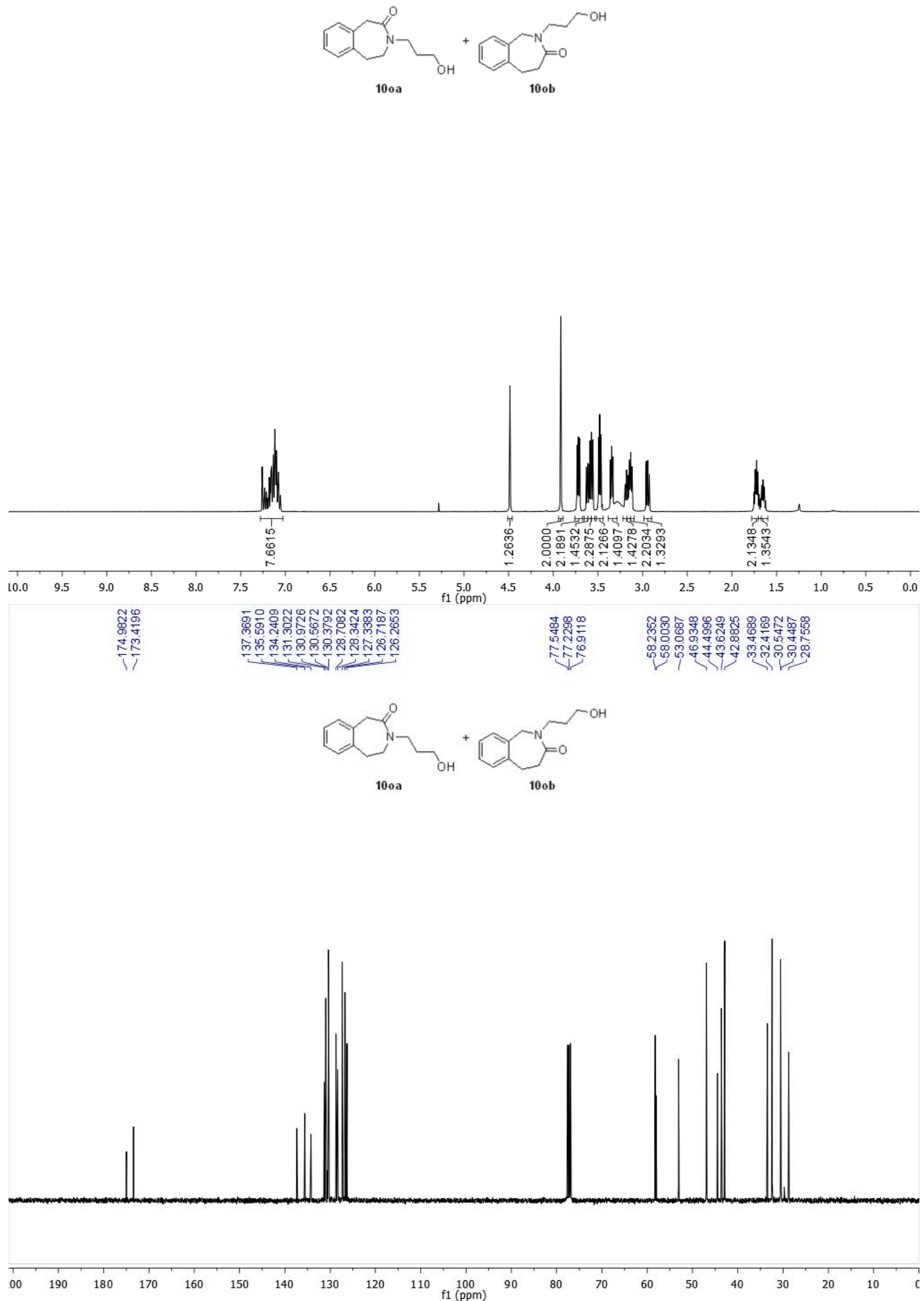
<smiles>O=C1Cc2ccccc2CN1CCCO</smiles>

10p
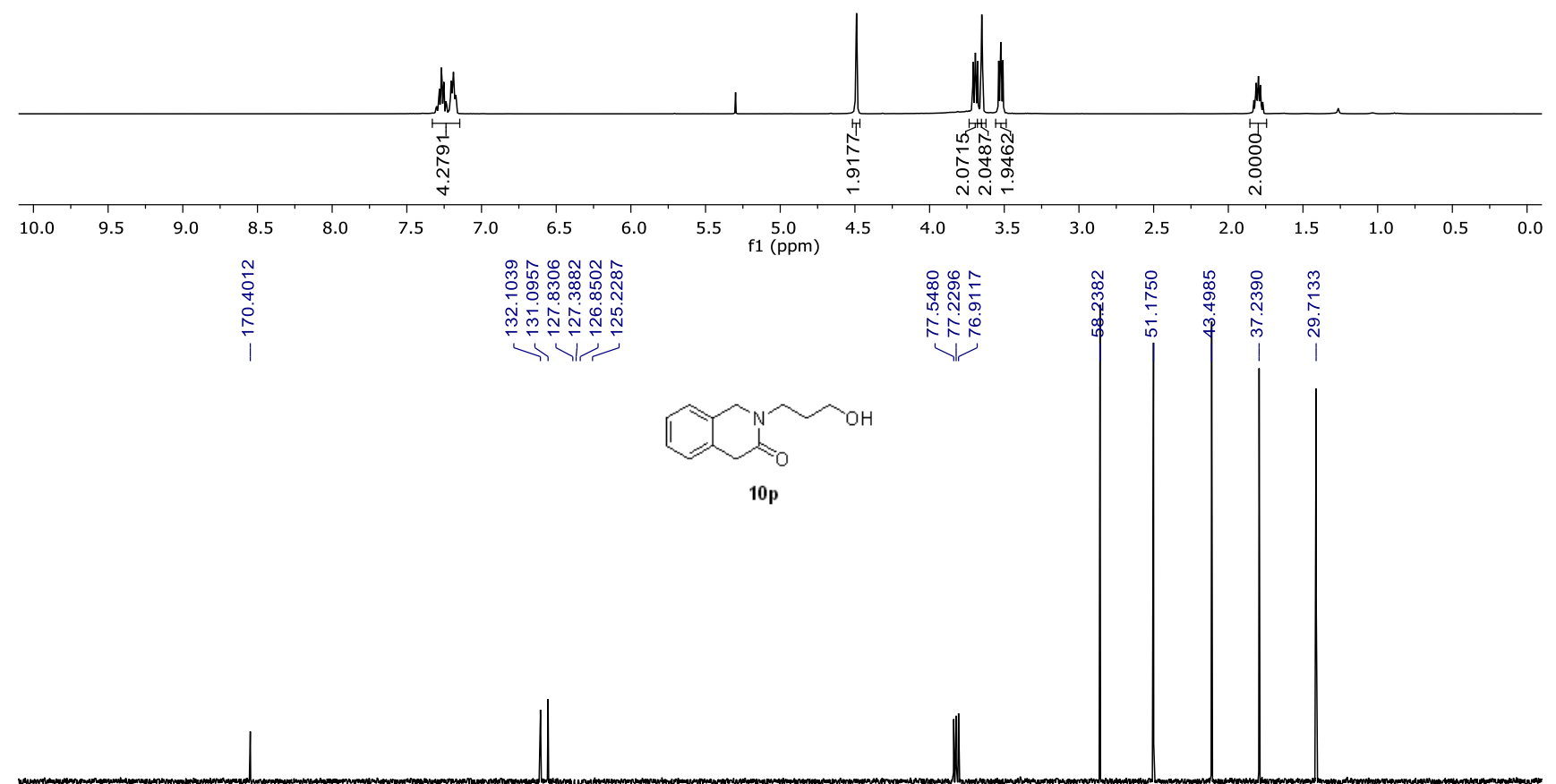

10p

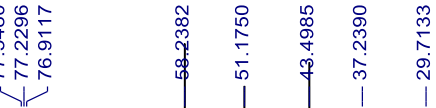

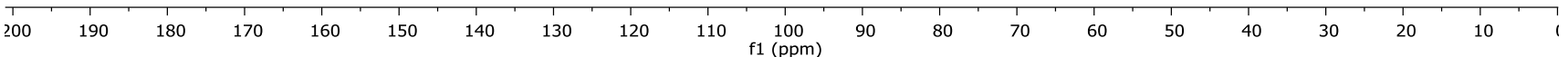



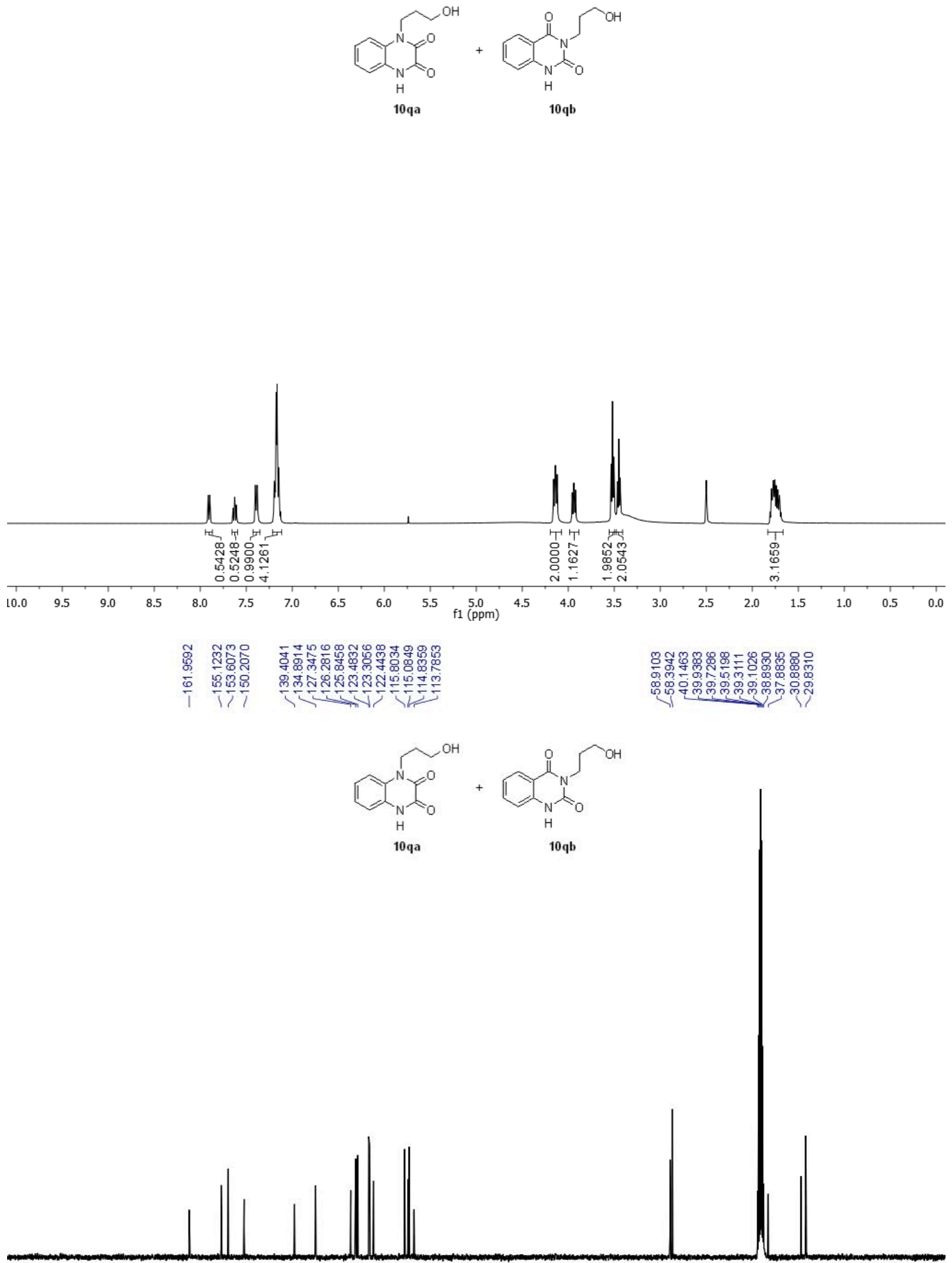

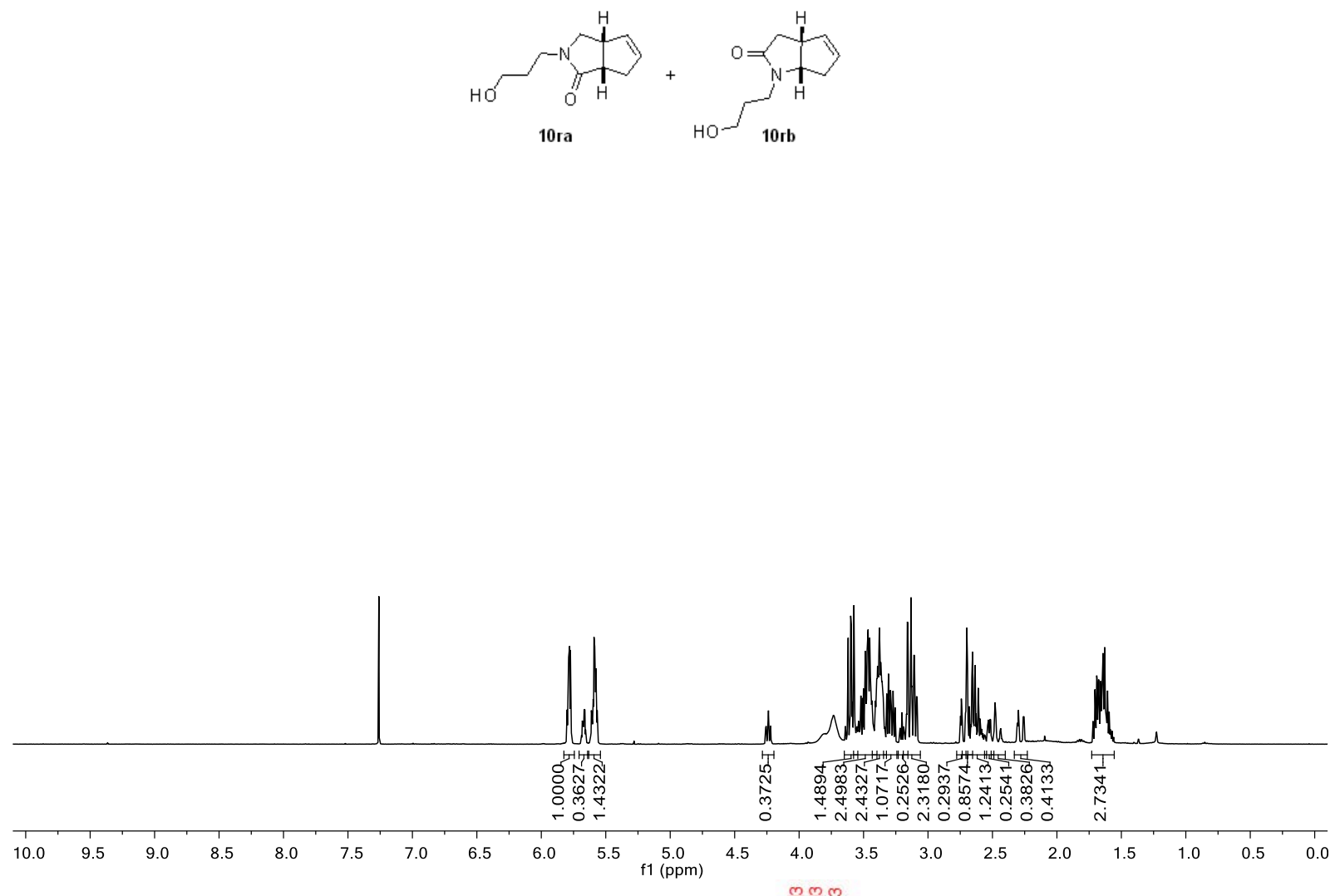

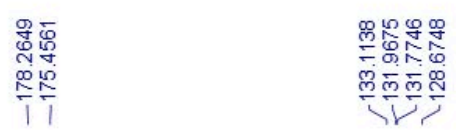

0
0

혀유을

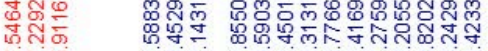

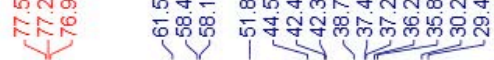
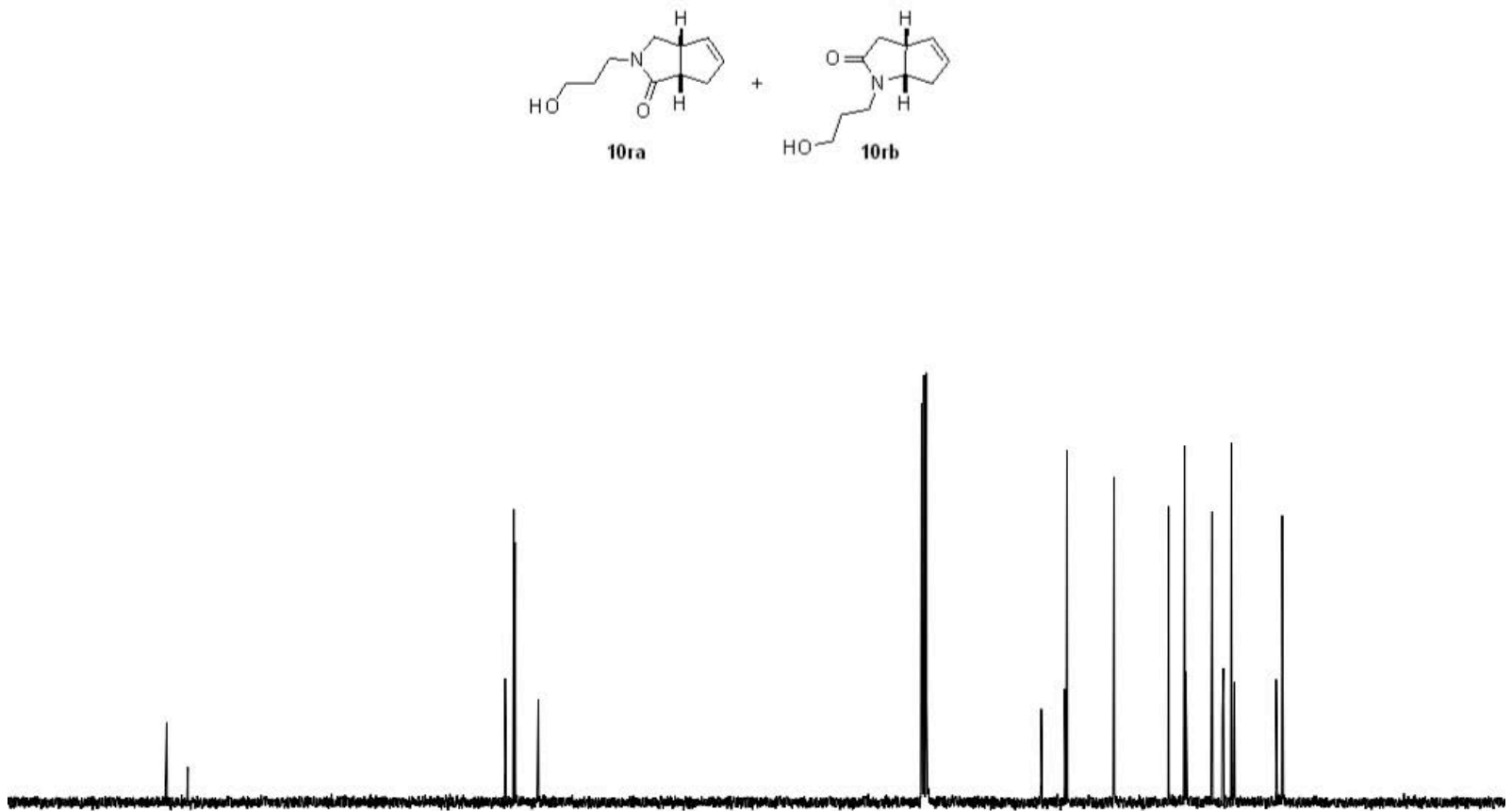

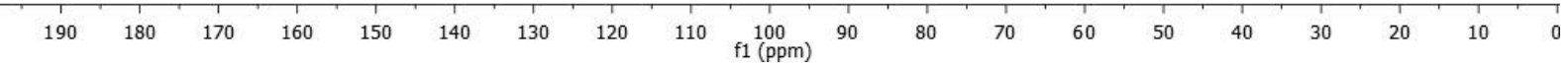



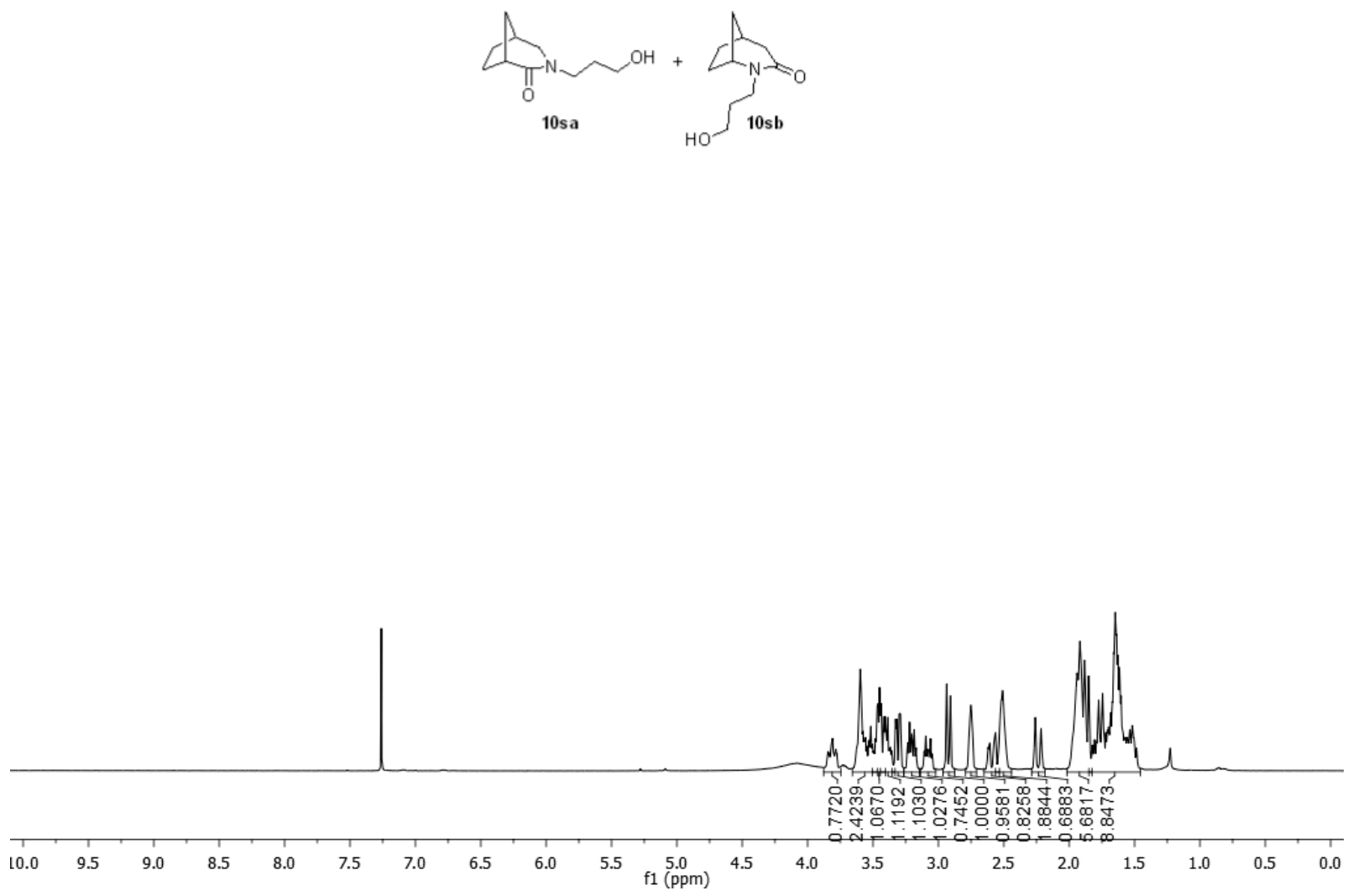

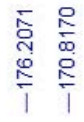
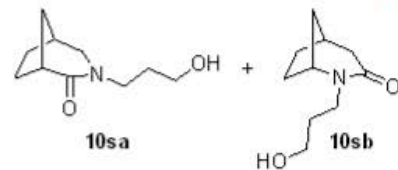

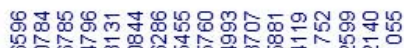

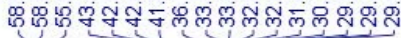

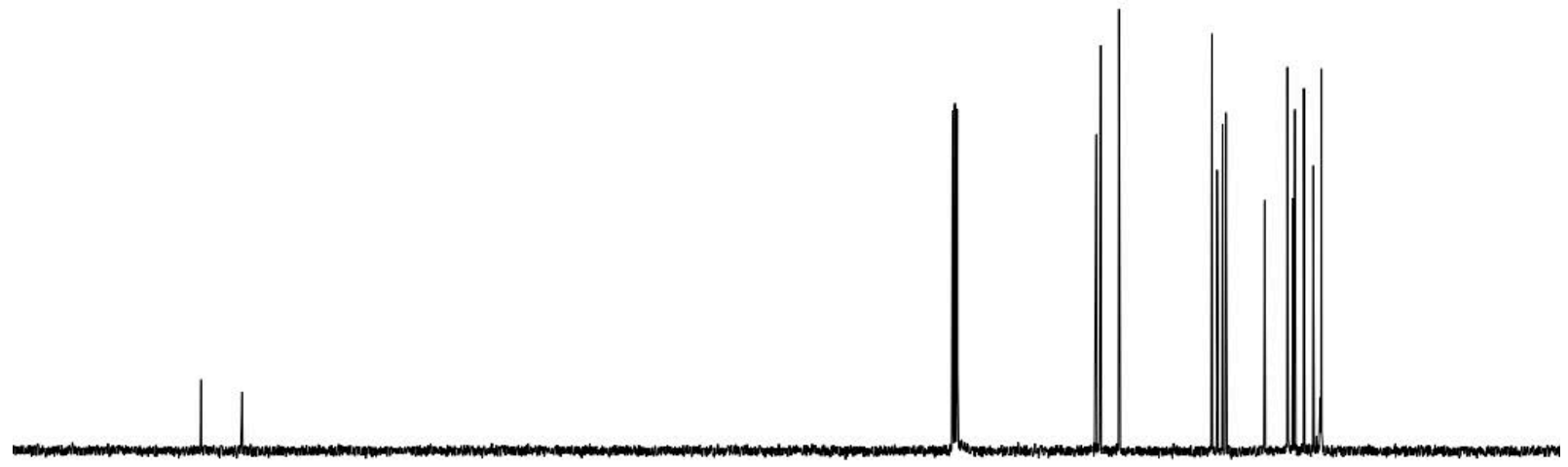



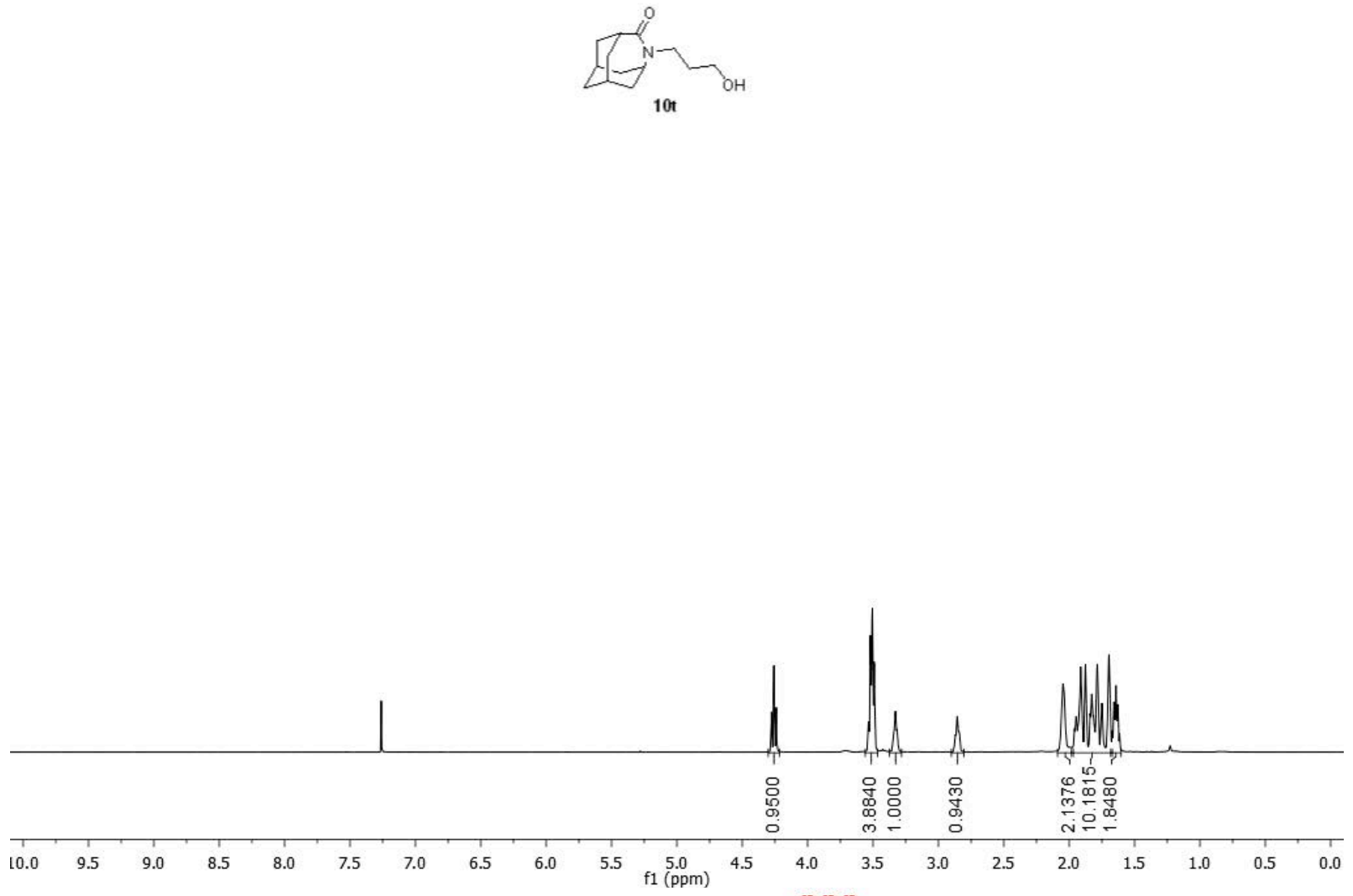

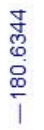

$\frac{m}{0} \frac{m}{0} \frac{m}{0}$

000

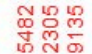

$\sqrt{n+\infty}$

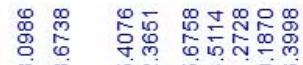

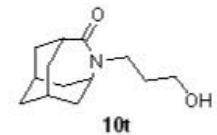

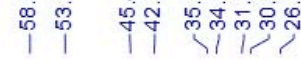

$10 \mathrm{t}$

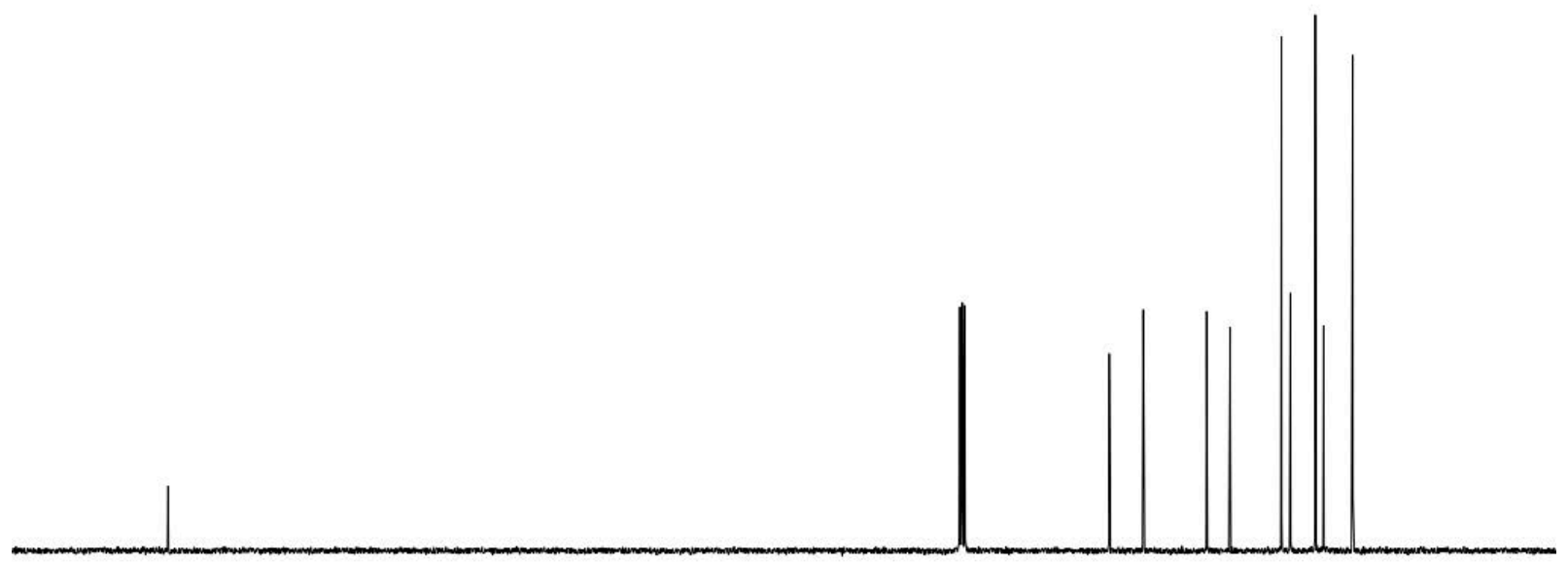

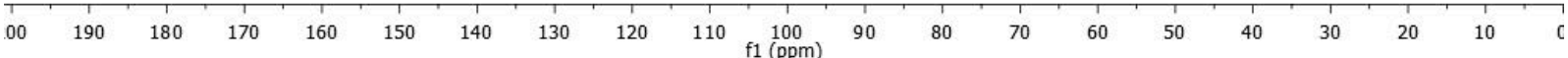




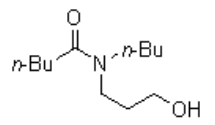

$10 \mathrm{k}$
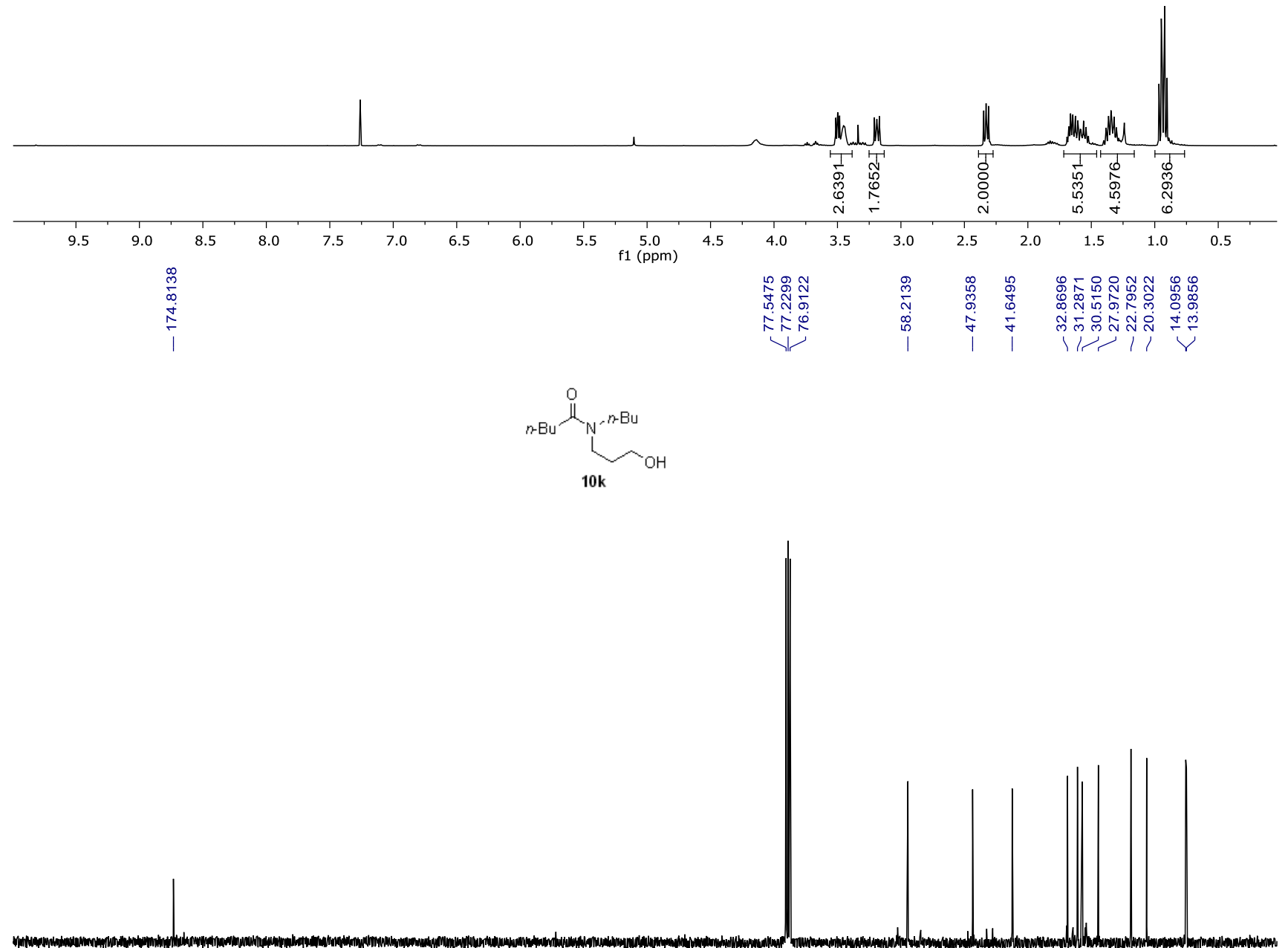

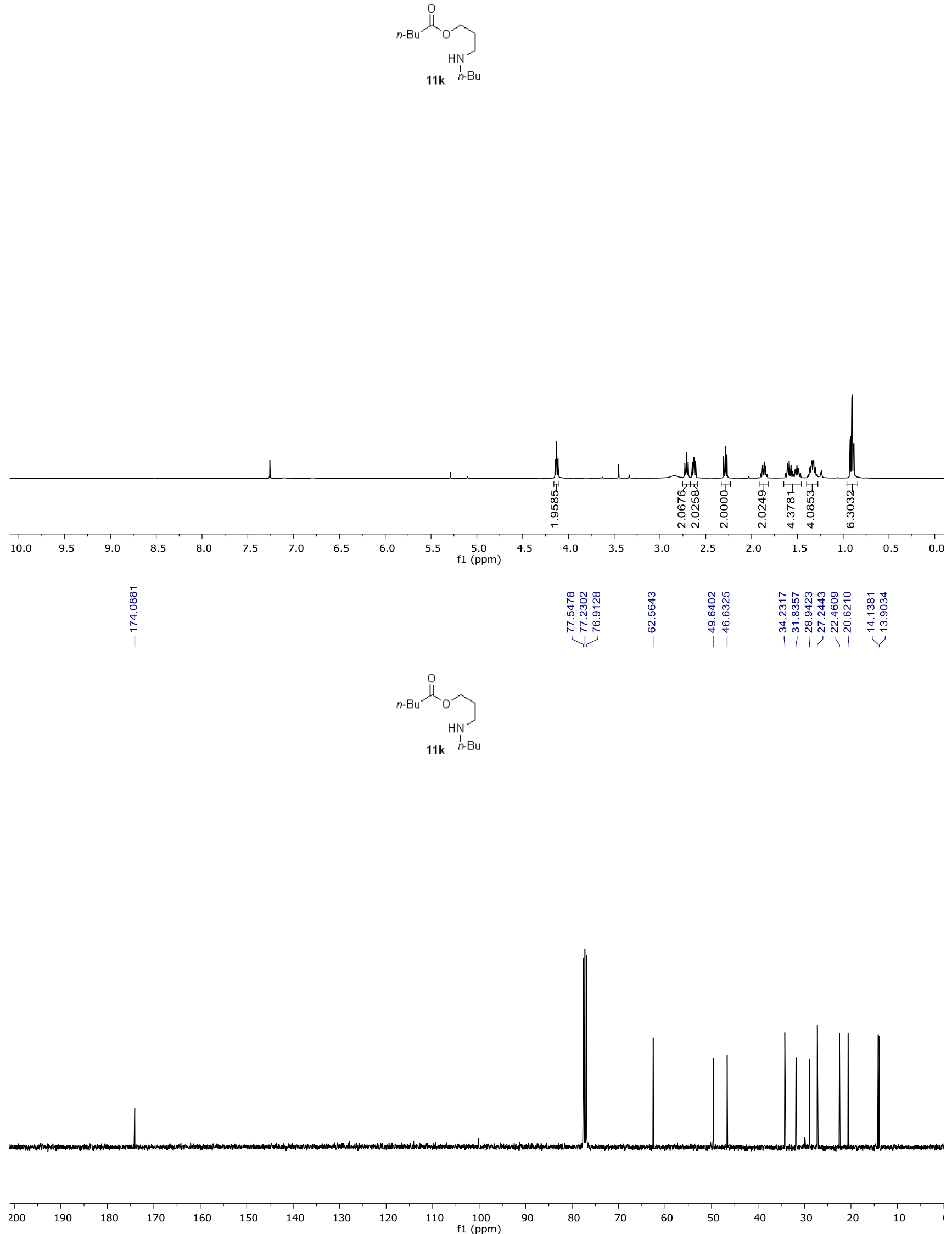